\title{
Box modelling of gas-phase atmospheric iodine chemical reactivity in case of a nuclear accident
}

\author{
Camille FORTIN, ,b,c $_{\text {alérie FEVRE-NOLLET, }}^{\mathrm{a}, \mathrm{c}}$ Frédéric COUSIN, ${ }^{\mathrm{b}, \mathrm{c}}$ Patrick \\ LEBEGUE, ${ }^{\mathrm{a}, \mathrm{c}}$ Florent LOUIS ${ }^{\mathrm{a}, \mathrm{c}, *}$
}

${ }^{\text {a }}$ Univ. Lille, CNRS, UMR 8522 - PC2A - Physicochimie des Processus de Combustion et de l’Atmosphère, 59000 Lille, France

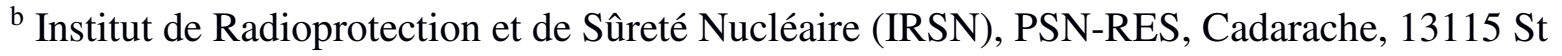
Paul Lez Durance, France

${ }^{\mathrm{c}}$ Laboratoire de Recherche Commun IRSN-CNRS-Lille "Cinétique Chimique, Combustion, Réactivité" (C3R), Cadarache, 13115 St Paul Lez Durance, France

Corresponding author : florent.louis@univ-lille.fr, Phone : (33)3203336332, Fax : (33)320436977

ORCID : Florent Louis 0000-0002-9533-557X

\begin{abstract}
A new atmospheric iodine gas-phase mechanism containing 248 reactions combined to the RACM air quality mechanism was implemented into a OD box model in order to evaluate the behaviour of iodine fission products released in the atmosphere. Different simulation scenarios were performed unravelling the influence of several parameters on the iodine speciation (season, day/night, low/medium/high iodine concentration release, nature of the injected iodine species). The main outcomes of our work are the following: (i) the $I_{2}$ reactivity is faster than that of $\mathrm{CH}_{3} \mathrm{I}$, (ii) iodine nitroxides $\mathrm{INO}_{\mathrm{x}}$ are mainly formed during night with low and medium releases in winter, (iii) iodine oxides are promoted with a high release of $\mathrm{I}_{2}$ during summer, (iv) the formation of organic iodine compounds can be highlighted only in the case of a low $\mathrm{I}_{2}$ release. In this work, $\mathrm{I}_{2} \mathrm{O}, \mathrm{I}_{2} \mathrm{O}_{5}$, and $\mathrm{HOIO}_{2}$ are the most abundant iodine oxides because their atmospheric removal pathways in the gas-phase are not yet known. The main iodinated organic species is $\mathrm{CH}_{3} \mathrm{OOI}$ arising from the reaction between $\mathrm{I}$ atom and the methyl peroxy radical. In case of a $\mathrm{CH}_{3} \mathrm{I}$ release, before the I atom formation, the reaction mechanism leads to either the formation or the destruction of several other organic iodinated compounds such as $\mathrm{CH}_{2} \mathrm{IOH}$ and $\mathrm{CH}_{2} \mathrm{IOO}$.
\end{abstract}


Keywords: Iodine; atmosphere; nuclear safety; 0D modelling

\section{Introduction}

Interest in iodine atmospheric chemistry is widely focused on the Marine Boundary Layer $(\mathrm{MBL})$ where are localized principal natural sources of molecular iodine $\left(\mathrm{I}_{2}\right)$ and iodocarbons (RI) such as $\mathrm{CH}_{3} \mathrm{I}$ and $\mathrm{CH}_{2} \mathrm{I}_{2}$ emitted by macro algae and phytoplankton (SaizLopez et al., 2006; von Glasow and Crutzen, 2003). Measurements conducted at Mace Head Research Station (Ireland) of the reactive iodine species (IO, OIO, $\mathrm{CH}_{3} \mathrm{I}$, and $\mathrm{I}_{2}$ ) detected mixing ratios around the pptV level reaching 100 pptV for the "hot-spots" (Seitz et al., 2010). The residence time of iodine species in the atmosphere is ranging from few days $\left(\mathrm{CH}_{3} \mathrm{I}\right)$ to seconds $\left(\mathrm{I}_{2}\right)$, it is linked directly to the solar photolysis level but it should be increased by formation of particles (McFiggans et al., 2004). Iodine species could affect the trace gas composition of the atmosphere by reaction with primary and secondary pollutants. Their impacts on ozone cycle and $\mathrm{NO}_{\mathrm{x}}$ regimes (Saiz-Lopez et al., 2014; Saiz-Lopez et al., 2012) or tropospheric $\mathrm{HOx}\left(\mathrm{OH}\right.$ and $\left.\mathrm{HO}_{2}\right)$ (Stone et al., 2018; Stone et al., 2012) have been significantly studied. Models are usually used to predict the impacts and behaviour of such species in the MBL or for global scale (Sherwen et al., 2016b) and various iodine chemistry schemes were developed and evaluated by comparison between field measurements and modelling (Sommariva et al., 2012).

Our work takes place in the field of the chemistry and transport modelling of fission products following a severe nuclear accident to predict the radiological consequences on the populations. Among fission products, iodine-131 whose half-life is equal to 8 days, is recognized as the main radionuclide due to its ability to accumulate in the thyroid gland, thereby increasing the effects of radiation on the human body. After the Fukushima accident, dispersion tools have been used and simulation results compared to measurement highlighted significant underestimating of the iodine radiation levels at the ground (Korsakissok et al., 2013). These differences could be explained by the fact that modelling tools used does not consider chemical transformations during transport in the atmosphere. The capture of gasphase species by atmospheric particles and the aerosol formation processes (nucleation, coagulation, ...) imply an increase of the radioactive deposition. It is very important to take into account the atmospheric iodine reactivity. For this purpose, we developed an exhaustive iodine gas-phase chemical mechanism, which is then combined to the air quality Regional Atmospheric Chemistry Mechanism (RACM) (Stockwell et al., 1997) and implemented on a 
OD box-model. The aim is to explore the variation of atmospheric concentrations and speciation of iodine modelled for various source term configuration (concentration and speciation, $\mathrm{CH}_{3} \mathrm{I}, \mathrm{I}_{2}, \mathrm{I}_{2} \mathrm{O}_{5}$ ) with realistic ambient conditions.

This paper is organized as follows. Section 2 described the methodology used to develop the gas-phase iodine mechanism as well as the 0D model for the evaluation of iodine reactivity as a function of various atmospheric conditions (photolysis conditions, night or day, winter or summer) and source term values. In section 3, the simulation set-up and input data is presented while section 4 contains the results and discussion.

\section{Iodine mechanism and OD model presentation}

\subsection{Iodine gas-phase mechanism}

Several OD or 3D modelling studies have already used an iodine gas-phase mechanism (Furneaux et al., 2010; Mahajan et al., 2009; McFiggans et al., 2000; Ordóñez et al., 2012; Pechtl et al., 2006; Saiz-Lopez et al., 2016; Saiz-Lopez et al., 2008; Sherwen et al., 2016a; Sherwen et al., 2016b; Sommariva et al., 2012). Table 1 lists the main informations about the number of organic and inorganic reactions involving the gas-phase iodine-containing species reactivity (thermal and photolysis). These mechanisms do not consider any organic reactivity and the extent of inorganic mechanism is variable.

The strategy used to constitute the reactional mechanism was to collect all gas-phase reactions involving iodine-containing species through the literature for a wide range of temperature from atmospheric to nuclear accident conditions (213 - $2500 \mathrm{~K})$. The mechanism does not contain reactions involving other halogen atoms such as chlorine and bromine. The elaboration of the mechanism has been done using the most recent evaluations available arising from the National Institute of Standards and Technology (NIST) kinetic database (Manion et al., 2015), International Union of Pure and Applied Chemistry (IUPAC), and Jet Propulsion Laboratory (JPL) evaluations (Burkholder et al., 2015).

For most of reactions, there is a good agreement with the recommended values of the thermokinetic parameters between the different databases. If it's not the case, the selected values correspond to the most recent experimental data. When reactions are missing, an examination of the theoretical works available in the literature (Bai et al., 2015; Canneaux et al., 2010; Cours et al., 2013; Fortin et al., 2018; Gómez Martin et al., 2013; Hammaecher et 
al., 2011; Kaltsoyannis and Plane, 2008; Khanniche et al., 2016b; Louis et al., 2011; Plane et al., 2006; Saiz-Lopez et al., 2016; Xerri et al., 2012) was performed and the corresponding thermokinetic data was added to the mechanism.

For an overall reaction involving several reaction pathways, the following procedure has been applied: i) select experimental data, ii) if branching ratios were not determined experimentally, select theoretical values if available. For example, in the case of the selfreaction of IO (R76, A. 2 for reaction number 76 in Appendix 2), the overall rate constant was taken from the work of Atkinson et al. (Atkinson et al., 2007) while the branching ratios were taken from the estimation made by Gómez-Martin et al. (Gómez Martín et al., 2007) for the four reaction pathways leading to $\mathrm{I}_{2}+\mathrm{O}_{2}, 2 \mathrm{I}+\mathrm{O}_{2}, \mathrm{I}+\mathrm{OIO}$, or $\mathrm{I}_{2} \mathrm{O}_{2}$.

The iodine gas-phase mechanism does not include reactions for which the thermokinetic data were assimilated to those of other halogen atoms. These estimations generated too much uncertainties to be taken into account. It was recently demonstrated for the reactions of halogen atoms with hydrogen peroxide (Fortin et al., 2018). A strong influence of the nature of the halogen atom on the rate constant at $298 \mathrm{~K}$ was shown with a change in the values of about twelve orders of magnitude when going from chlorine to iodine.

The updated iodine gas-phase mechanism contains 231 reactions (139 and 92 for organic and inorganic species), 17 photolysis reactions (6 and 11 for organic and inorganic species). This mechanism is presented in Appendices A1-A4 with the corresponding references for each reaction. It is then combined to the air quality RACM mechanism (Stockwell et al., 1997), which contains 237 reactions (23 photolysis reactions) involving 77 species. The aggregation procedure developed for organic iodine species is the same as RACM mechanism organic section (see IRACM class in Table 2 and corresponding IRACM reactions in Appendices A1 and A3).

\subsection{Model presentation}

The iodine mechanism described in previous section has been implemented in the ASTEC (Accident Source Term Evaluation Code) software dedicated to model a nuclear severe accident (Chatelard et al., 2016), which was developed by the French Nuclear Safety Institute (IRSN). It is divided into several modules taking into account each possible phenomenon that can occur during the accident. Iodine is the main important radio-element due to its radio-toxicity. Therefore, iodine chemistry is an important process, which has to be 
modelled during a severe accident. A dedicated module called SOPHAEROS (Cousin et al., 2013) is implemented in the ASTEC code to model the iodine chemistry.

The SOPHAEROS module used a OD approach to compute iodine chemistry. The implementation of iodine mechanism in SOPHAEROS has to be sufficiently flexible due to on-going researches on iodine behaviour during a severe accident (Bosland et al., 2010; Cantrel et al., 2014; Girault et al., 2012). The 0D box model is defined with a fixed volume. The inside conditions are characterized by different parameters: temperature, pressure, volatile organic compounds (VOC) and $\mathrm{NO}_{\mathrm{x}}$ emissions. The model computes the mass evolution solving a differential equation for each species involved in the mechanism using a Newton-Raphson algorithm.

\section{Input data and Simulation set-up}

\subsection{Air quality conditions}

All simulations were made at constant atmospheric temperature $(298 \mathrm{~K})$ and pressure (1.013 bar). The box volume is fixed to $10000 \mathrm{~m}^{3}$. To take into account the reactivity of iodine with atmospheric species, initial ambient concentrations were fixed for $\mathrm{H}_{2}, \mathrm{H}_{2} \mathrm{O}, \mathrm{H}_{2} \mathrm{O}_{2}$, $\mathrm{OH}, \mathrm{HO}_{2}, \mathrm{~N}_{2}$, and $\mathrm{O}_{2}$ (see Table 3 and corresponding references therein). We modelled the temporal variation of $\mathrm{NO}_{\mathrm{x}}$ and Volatile Organic Compounds (VOC) emissions based on Kuhn et al. values (Kuhn et al., 1998) given in Table 4 and Menut et al. temporal profile (Menut et al., 2013). The 67 VOC species proposed by Kuhn et al. (Kuhn et al., 1998) were aggregated into the RACM VOC chemical scheme whose obtained speciation is given in Table 5. Iodine release was made after a spin-up time necessary to achieve $\mathrm{O}_{3}$ and $\mathrm{VOC} / \mathrm{NO}_{\mathrm{x}}$ realistic conditions (from 12 to 24 hours depending on the scenario season). Network station measurements in the North of France (15 stations) were chosen as reference for $\mathrm{O}_{3}$ concentration, $\mathrm{NO}_{\mathrm{x}}$ speciation ( $\mathrm{NO}: 98 \%, \mathrm{NO}_{2}: 2 \%$ ), and $\mathrm{VOC} / \mathrm{NO}_{\mathrm{x}}$ ratio. The ozone concentration values range between 1 and $92 \mu \mathrm{g} \mathrm{m}^{-3}$ while the $\mathrm{VOC} / \mathrm{NO}_{\mathrm{x}}$ ratio vary from to 3 to 10. Both depends on month and time of the day.

For photolysis rates, actinic flux was calculated with the Tropospheric Ultraviolet and Visible (TUV) radiation model, which was available from the National Center for Atmospheric Research (NCAR) for clear sky, beginning of January and August, latitude $51^{\circ}$ $2^{\prime} 3,725^{\prime \prime} \mathrm{N}$ and longitude $2^{\circ} 22^{\prime} 36,394^{\prime \prime} \mathrm{E}$. The cross sections and quantum yields used are detailed in Appendices A3-A4 for organic and inorganic species, respectively. 


\subsection{Iodine speciation and amount}

During the Chernobyl accident, it was estimated around $1760 \mathrm{PBq}$ of ${ }^{131} \mathrm{I}$ isotope released to environment (IAEA, 2006), which corresponds to $380 \mathrm{~g}$ of ${ }^{131} \mathrm{I}$. Because the ${ }^{131} \mathrm{I}$ isotope is a small fraction of total iodine isotope inventory, it can be estimated that few kilograms of stable and radioactive iodine isotopes were released.

In the same way, during the Fukushima accident, (Katata et al., 2015) or (Lebel et al., 2016) estimated around $160 \mathrm{PBq}$ of $\mathrm{I}^{131}$ isotope released. Saunier and co-workers (Saunier et al., 2013) made a lower evaluation around $100 \mathrm{PBq}$. It corresponds to a value varying between 20 and $30 \mathrm{~g}$ of ${ }^{131} \mathrm{I}$ isotope. The global Fukushima release can be estimated around 1 or $2 \mathrm{~kg}$ of stable and radioactive iodine isotopes. One can notice that at the source level the nuclear release infers higher mass of iodine than in natural marine conditions (Saiz-Lopez et al., 2012).

Obviously, the main parameter for iodine chemistry in the atmosphere is not the total amount of iodine, which can be released during an accident but the initial iodine concentration and speciation in the puff during the release. This speciation and concentration are not easy to define near the nuclear power plant. Iodine speciation in the containment depends on several parameters such as temperature, water, and gaseous dose rate, sump $\mathrm{pH}$, organic compounds (Cantrel et al., 2014; Girault et al., 2012). The speciation is governed by the competition between the iodine formation/desorption and destruction/adsorption processes that take place in the atmosphere of the containment and in the water. The main iodine species formed are inorganic iodine considered as molecular iodine, organic iodine represented by the most volatile one, the iodomethane $\mathrm{CH}_{3} \mathrm{I}$ and iodine oxide. With these three kind of species, taking into account several radiolytic and thermal reactions, it is possible in a severe accident code to model integral experiments such as ones from the Phebus FP (Fission Product) Program (Girault et al., 2012). The iodine oxides are known to nucleate and form particles (Hoffmann et al., 2001; Saunders and Plane, 2006). That's why they are considered as aerosols in the atmosphere of the nuclear power plant.

The repartition of these three species is not constant during the accident. To cover all the initial speciation, which can be released in the environment, it is necessary to consider a mix of organic, inorganic, and iodine oxide in the puff. To define representative iodine concentration during the release, it is necessary to estimate the fission product behaviour during a severe accident from the release from fuel pellets to the release in the environment. This behaviour is directly dependent on thermal-hydraulics conditions in the nuclear power plant, leakage to environment, fission product transport, and chemistry. 
The aim of the ASTEC code is to model all these phenomena to give an assessment of the global release called source term. A lot of uncertainties have to be considered in this source term evaluation. (Chevalier-Jabet et al., 2014) studied the source term uncertainties regarding iodine chemistry mechanism in the containment with the ASTEC code on a severe accident transient on a Pressurized Water Reactor (PWR) of 1300 MWe. In the containment, the iodine mass can vary between 10 to $10^{-5} \mathrm{~kg}$, which corresponds to $10^{-4}$ to $10^{-10} \mathrm{~kg} \mathrm{~m}^{-3}$ assuming a reactor building volume around $80000 \mathrm{~m}^{3}$. In a nuclear power plant, several aerosol and gas filters are used to reduce the release during the accident. Regarding the physical form of iodine (aerosol, gaseous molecular iodine, gaseous organic iodine), filter efficiency can vary a lot. A good decontamination factor can be estimated around 1000 whereas a poor decontamination factor is around 10. If these values are applied to iodine concentration in the containment, the iodine quantity released in the atmosphere during an accident can be evaluated between $10^{-5}$ to $10^{-13} \mathrm{~kg} \mathrm{~m}^{-3}$. This range of concentration can be reduced if it is assumed high efficiency of the filter for aerosol. This form of iodine is predominant at the beginning of the release (Chevalier-Jabet et al., 2014), which leads to a maximal level around $10^{-7} \mathrm{~kg} \mathrm{~m}^{-3}$. At long term, after aerosol deposition, iodine in the atmosphere is due to gaseous chemistry and compounds transferred from water to gas phase (Girault et al., 2012). Iodine gaseous species are more difficult to trap but this low efficiency can be applied to lower concentration. It leads to a minimal level around $10^{-11} \mathrm{~kg} \mathrm{~m}^{-3}$. Iodine source term was injected in the box during one second with mass flow rates $10^{-7}$ to $10^{-11} \mathrm{~kg}$ $\mathrm{m}^{-3} \mathrm{~s}^{-1}$.

\subsection{Model scenarios}

We considered various representative scenarios as described in Table 6. Parameters studied are high $(\mathrm{H})$, medium $(\mathrm{M})$, and low $(\mathrm{L})$ levels for source term quantity $\left(10^{-7}, 10^{-9}\right.$, and $10^{-11} \mathrm{~kg} \mathrm{~m}^{-3}$, respectively), $\mathrm{I}_{2}$ or $\mathrm{CH}_{3} \mathrm{I}$ iodine speciation, nightly ( $\mathrm{N}$ ) or daily (D) release, and month of the year, January for winter (W) or August for summer (S). In the model, source term concentrations are given in pptV. For example, a medium release value $\left(10^{-9} \mathrm{~kg} \mathrm{~m}^{-3}\right)$ corresponds to either $98 \mathrm{pptV}$ for $\mathrm{I}_{2}$ or $196 \mathrm{pptV}$ for $\mathrm{CH}_{3} \mathrm{I}$. 


\section{Results and discussion}

Iodinated compounds are grouped into five families: iodine nitroxides $I N O_{x}$ (INO, $\mathrm{INO}_{2}, \mathrm{IONO}_{2}$ ), iodine organic species $I_{-} O R G$. (molecules and radicals), iodine oxides $I_{x} O_{y}$ $\left(\mathrm{I}_{2} \mathrm{O}, \mathrm{I}_{2} \mathrm{O}_{2}, \mathrm{I}_{2} \mathrm{O}_{3}, \mathrm{I}_{2} \mathrm{O}_{4}, \mathrm{I}_{2} \mathrm{O}_{5}\right.$, and its hydrated form $\left.\mathrm{HOIO}_{2}\right)$, inorganic iodine species I_INORG. ( $\left.\mathrm{I}_{2}, \mathrm{HI}, \mathrm{HOI}\right)$, and iodine radicals $R \_I N O R G$. (I, IO, OIO).

\subsection{Iodine nitroxides formation}

\subsubsection{Iodine nitroxides regarding $\mathrm{I}_{2}$ or $\mathrm{CH}_{3} \mathrm{I}$ release}

Figure 1 displays the temporal iodine speciation obtained with January photolysis conditions for low, medium, and high daily $\mathrm{I}_{2}$ or $\mathrm{CH}_{3} \mathrm{I}$ release. The $I N O_{x}$ family is more important and faster produced with an $\mathrm{I}_{2}$ release. At midnight, 17 hours after the release, $97 \%$ of iodine nitroxides are formed with an $\mathrm{I}_{2}$ release of $0.98 \mathrm{pptV}$ (Fig. 1 (a)) compared to only $21 \%$ with the $\mathrm{CH}_{3} \mathrm{I}$ release of $1.96 \mathrm{pptV}$ (Fig. 1 (b)). The iodine species released have an important impact on the iodine nitroxides formation rate. For example, 14 hours are needed for $\mathrm{CH}_{3} \mathrm{I}$ release to reach the same level of iodine nitroxides $(20 \%)$ reached in one hour with an $I_{2}$ release (Fig. 1 (a) and (b)). The same evolution is noticed for simulations with medium release (Fig. 1 (c) and (d)).

The delay to obtain $I N O_{x}$ compounds with the $\mathrm{CH}_{3}$ I release can be explained regarding the kinetic scheme presented in Fig. 2. The $I_{2}$ photolysis is faster by comparison to that of $\mathrm{CH}_{3} \mathrm{I}$. As seen in Appendices 3 and 4, the $\mathrm{I}_{2}$ photolysis rate constant $J$ is about 10000 times greater than the one of $\mathrm{CH}_{3} \mathrm{I}$. It can be also noticed that more reaction steps are needed in the case of the $\mathrm{CH}_{3} \mathrm{I}$ release:

$$
\begin{aligned}
& \mathrm{CH}_{3} \mathrm{I}+\mathrm{h} v \rightarrow \mathrm{CH}_{3}+\mathrm{I} \\
& \mathrm{CH}_{3} \mathrm{I}+\mathrm{NO}_{3} \rightarrow \mathrm{CH}_{2} \mathrm{I}+\mathrm{HNO}_{3} \\
& \mathrm{CH}_{2} \mathrm{I}+\mathrm{O}_{2} \rightarrow \mathrm{CH}_{2} \mathrm{IO}_{2} \\
& \mathrm{CH}_{2} \mathrm{I}+\mathrm{O}_{2} \rightarrow \mathrm{HCH}(\mathrm{O})+\mathrm{IO} \\
& \mathrm{CH}_{2} \mathrm{IO}_{2}+\mathrm{CH}_{2} \mathrm{IO}_{2} \rightarrow 0.5 \mathrm{CH}_{2} \mathrm{IOH}+0.5 \mathrm{HC}(\mathrm{O}) \mathrm{I}+\mathrm{CH}_{2} \mathrm{IO}+\mathrm{O}_{2} \\
& \mathrm{CH}_{2} \mathrm{IOH}+\mathrm{h} v \rightarrow \mathrm{I}+\mathrm{CH}_{2} \mathrm{OH} \\
& \mathrm{I}+\mathrm{O}_{3} \rightarrow \mathrm{IO}+\mathrm{O}_{2} \\
& \mathrm{IO}+\mathrm{NO}_{2}(+\mathrm{M}) \rightarrow \mathrm{IONO}_{2}(+\mathrm{M}) \\
& \text { compared to the } \mathrm{I}_{2} \text { release: } \\
& \mathrm{I}_{2}+\mathrm{h} v \rightarrow \mathrm{I}+\mathrm{I} \\
& \mathrm{I}+\mathrm{O}_{3} \rightarrow \mathrm{IO}+\mathrm{O}_{2}
\end{aligned}
$$


For the $\mathrm{CH}_{3} \mathrm{I}$ release, iodoalkanes and $\mathrm{CH}_{2} \mathrm{IOH}$ are treated as I_HC3 species in the IRACM aggregation scheme. The atmospheric degradation of iodomethanol can be considered by analogy to another iodinated alkane compound. Similar reactions can be expected and are represented by dashed red lines in the Fig. 2 (b). For example, the $\mathrm{CH}_{2} \mathrm{IOH}$ photolysis is expected to be close to that of an iodinated propane $\mathrm{C}_{3} \mathrm{H}_{7} \mathrm{I}$, which belongs to the same I_HC3 family.

In case of an high release (Fig. 1 (e) and (f)), the iodine concentration in the box is greater than the concentration of atmospheric pollutants $\left(\mathrm{O}_{3}, \mathrm{NO}_{\mathrm{x}}\right)$. These pollutants are quickly consumed to form $I N O_{x}$ and $I_{x} O_{y}$ species. Their quantity depends only on the $\mathrm{O}_{3}$ and $\mathrm{NO}_{\mathrm{x}}$ concentrations. After 24 hours, about $85 \%$ of iodine release remains in the simulation. To get a more precise behaviour with high release conditions, the use of a Chemical Transport Model (CTM) or Puff model is needed.

\subsubsection{Iodine nitroxides speciation}

Fig. 3 presents the temporal $\mathrm{INO}_{2}$ and $\mathrm{IONO}_{2}$ mass speciation for the different scenarios. Nitrosyl Iodide (INO) is not represented on the Fig. 3 because its speciation is less than $0.01 \%$ of the total $I N O_{x}$. Regardless of the nature of the injected iodinated compound $\left(\mathrm{I}_{2}\right.$ or $\mathrm{CH}_{3} \mathrm{I}$ ), iodine nitrate $\left(\mathrm{IONO}_{2}\right)$ is the main $I N O_{x}$ species formed (around $99.9 \%$ ) for low and medium releases (Fig. 3 (a), (b), (c), and (d)). The $\mathrm{INO}_{2}$ species is produced during the day and can represent up to $5 \%$ of the total INOx (Fig. 3 (c)). A day/night cycle appears for both species. This cycle is more significant in the case of high releases (Fig. 3 (e) and (f)).

Fig. 4 presents the iodine reaction scheme for an release of $98 \mathrm{pptV}$ of $\mathrm{I}_{2}$ during day (Fig. 4 (a)) and night (Fig. 4 (b)) in January.

During the night, main reactions are driven by the nitrate radical $\left(\mathrm{NO}_{3}\right) . \mathrm{IONO}_{2}$ is mainly formed by the following reaction

$\mathrm{I}_{2}+\mathrm{NO}_{3} \rightarrow \mathrm{I}+\mathrm{IONO}_{2}$

During the day, the photolysis reaction of iodine nitrate

$$
\mathrm{IONO}_{2}+\mathrm{h} v \rightarrow 0.5 \mathrm{I}+0.5 \mathrm{NO}_{3}+0.5 \mathrm{IO}+0.5 \mathrm{NO}_{2}
$$

leads to the $\mathrm{IONO}_{2}$ destruction. The formation of $\mathrm{INO}_{2}$ by the reaction

$\mathrm{I}+\mathrm{NO}_{2}(+\mathrm{M}) \rightarrow \mathrm{INO}_{2}(+\mathrm{M})$

can be significant within the $I N O_{x}$ family. 
Since the day/night cycle has an impact on the iodine nitroxide formation, obviously, the month of release will affect the $I N O_{x}$ reactivity. Fig. 5 displays the family evolution of iodine for a medium release of $I_{2}$ during day and night in winter and summer. It can be noted that, independently of the season release, there is a rapid formation of nitroxide species during the day. Afterwards, their destruction occurs due to photolysis reactions. Obviously, photolysis reactions are more efficient in summer, which leads finally to a lower amount of iodine nitroxide species $22 \%$ in summer after 24 hours (Fig. 5 (c)) compared to winter $96 \%$ (Fig. 5 (a)). During night, the season does not affect strongly the $I N O_{x}$ formation. Reactive schemes obtained for summer test cases are very similar to those of winter presented in Fig. 4.

\subsection{Iodine oxides formation}

\subsubsection{Iodine oxides regarding $\mathrm{I}_{2}$ or $\mathrm{CH}_{3} \mathrm{I}$ release}

Fig. 6 presents the family mass evolution of iodine in the case of $\mathrm{I}_{2}$ and $\mathrm{CH}_{3} \mathrm{I}$ medium release at 4 am in August. At noon, 8 hours after release, $I_{x} O_{y}$ family represents $74 \%$ of the total iodine compared to $4 \%$ in case of the $\mathrm{CH}_{3} \mathrm{I}$ release. The iodine oxide formation raises as the iodine injected quantity increases for low and medium release scenarios $\left(\mathrm{I}_{2}\right.$ or $\left.\mathrm{CH}_{3} \mathrm{I}\right)$. It is worth noticing that iodine oxide species are not present in such quantities in winter with low and medium releases $(<1 \%)$. The iodine reaction scheme corresponding to the previous simulation scenario is depicted in Fig. 7 for $\mathrm{I}_{2}$ (a) and $\mathrm{CH}_{3} \mathrm{I}$ releases (b). As already discussed in the iodine nitroxides formation (see 4.1.1.), more reaction steps are needed to get the key precursor species of iodine oxides $\left(R \_I N O R G\right.$.) with the $\mathrm{CH}_{3} \mathrm{I}$ release.

$\mathrm{I}_{2} \mathrm{O}_{3}$ is considered as one of the gas-phase main precursor of higher iodine oxides species, his formation is obtained by either the biradicalar reaction

$\mathrm{IO}+\mathrm{OIO} \rightarrow \mathrm{I}_{2} \mathrm{O}_{3}$

or the $\mathrm{I}_{2} \mathrm{O}_{2}$ oxidation by ozone

$\mathrm{I}_{2} \mathrm{O}_{2}+\mathrm{O}_{3} \rightarrow \mathrm{I}_{2} \mathrm{O}_{3}+\mathrm{O}_{2}$

In the $\mathrm{CH}_{3} \mathrm{I}$ release scenario, only the reaction of iodine monoxide with iodine dioxide ( $\mathrm{R} 83$, A.2) is significant.

\subsubsection{Iodine oxides speciation}

Fig. 8 presents the iodine oxides speciation corresponding to the evolution and simulation conditions presented in Fig. 6. Regardless of the nature of the injected iodine $\left(\mathrm{I}_{2}\right.$ or $\mathrm{CH}_{3} \mathrm{I}$ ), the same iodine oxides species are formed. The major species are $\mathrm{I}_{2} \mathrm{O}, \mathrm{I}_{2} \mathrm{O}_{5}$, and the iodic acid $\mathrm{HOIO}_{2}$ while $\mathrm{I}_{2} \mathrm{O}_{2}, \mathrm{I}_{2} \mathrm{O}_{3}$, and $\mathrm{I}_{2} \mathrm{O}_{4}$ are present in less quantities. These latter act as 
intermediate compounds leading to the formation of gas-phase $\mathrm{I}_{2} \mathrm{O}_{5}$ and $\mathrm{HOIO}_{2}$. To the best of our knowledge, there are no literature data concerning the atmospheric fate of $\mathrm{I}_{2} \mathrm{O}$ by photolysis or reactions with other iodine oxides. Its removal from the atmosphere is only assessed by the reaction with I atoms

$\mathrm{I}+\mathrm{I}_{2} \mathrm{O} \rightarrow \mathrm{I}_{2}+\mathrm{IO}$

These facts can explain the high level of $\mathrm{I}_{2} \mathrm{O}$ concentrations found in our simulations.

The comparison between low, medium, and high $\mathrm{I}_{2}$ release highlights the increase of $\mathrm{I}_{2} \mathrm{O}$ formation by the reaction

$\mathrm{I}+\mathrm{IO} \rightarrow \mathrm{I}_{2} \mathrm{O}$

In the high concentration scenario, there are two distinct reaction paths explaining the formation of major iodine oxide species

path I:

$\mathrm{IO}+\mathrm{IO} \rightarrow \mathrm{I}+\mathrm{OIO}$

$\mathrm{IO}+\mathrm{OIO} \rightarrow \mathrm{I}_{2} \mathrm{O}_{3}$

path II:

$\mathrm{IO}+\mathrm{IO} \rightarrow \mathrm{I}_{2} \mathrm{O}_{2}$

$\mathrm{I}_{2} \mathrm{O}_{2}+\mathrm{O}_{3} \rightarrow \mathrm{I}_{2} \mathrm{O}_{3}+\mathrm{O}_{2}$

followed by the same final reaction scheme:

$\mathrm{I}_{2} \mathrm{O}_{3}+\mathrm{O}_{3} \rightarrow \mathrm{I}_{2} \mathrm{O}_{4}+\mathrm{O}_{2}$

$\mathrm{I}_{2} \mathrm{O}_{4}+\mathrm{O}_{3} \rightarrow \mathrm{I}_{2} \mathrm{O}_{5}+\mathrm{O}_{2}$

$\mathrm{I}_{2} \mathrm{O}_{5}+\mathrm{H}_{2} \mathrm{O} \rightarrow 2 \mathrm{HOIO}_{2}$

The atmospheric removal of iodine pentoxide and its hydrated form $\mathrm{HOIO}_{2}$ is not well evaluated in the literature with the exception of the reaction of $\mathrm{OH}$ radicals with iodic acid for which a recent theoretical study was performed in our group (Khanniche et al., 2017) enabling to get the kinetic parameters over a wide temperature range $(250-2500 \mathrm{~K})$. This work showed that the H-abstraction pathway was the most important and the value of the rate constant at $298 \mathrm{~K}$ was about $10^{-17} \mathrm{~cm}^{3}$ molecule ${ }^{-1} \mathrm{~s}^{-1}$. It was concluded that $\mathrm{HOIO}_{2}$ could be transported to different places around the Earth. Recent field measurements in various locations confirmed the presence of iodic acid (Kürten et al., 2016; Rissanen et al., 2019; Sipilä et al., 2016). 
Other atmospheric removal reactions of $\mathrm{I}_{2} \mathrm{O}_{5}$ or $\mathrm{HOIO}_{2}$ such as nucleation or condensation onto aerosols have to be taken into account (Hoffmann et al., 2001; Saiz-Lopez et al., 2012; Sipilä et al., 2016) when using a multiphase modelling approach.

\subsubsection{Photolysis impact on iodine oxides formation}

Iodine oxides are produced faster in summer than in winter. At noon, $20 \%$ of iodine corresponds to iodine oxides in winter (see WDM-I $\mathrm{I}_{2}$ scenario, Fig. 5 (a)) while this proportion reaches about $60 \%$ in summer (see SDM-I $\mathrm{I}_{2}$ scenario, Fig. 5 (c)). Similar trends are found when injecting organic iodine. These remarks can be attributed to both photolysis conditions and ozone production with an average value varying from 30 to $50 \mu \mathrm{g} \mathrm{m}^{-3}$ in winter and summer, respectively.

There is no impact of the season release on the iodine oxides speciation. Same major species are found $\left(\mathrm{I}_{2} \mathrm{O}, \mathrm{I}_{2} \mathrm{O}_{5}\right.$ and $\left.\mathrm{HOIO}_{2}\right)$.

\subsection{Organic iodine}

\subsubsection{Organic iodine formation with $\mathrm{I}_{2}$ release}

In order to evaluate the interaction between iodine and atmospheric VOC, we study the scenario of an $I_{2}$ release as depicted in the Fig. 1 at 7 am in January. In the case of the WDH- $I_{2}$ scenario (high release of $I_{2}$ ), the formation of organic iodine species is found negligible while it can reach a few $\%$ in the WDL-I $\mathrm{I}_{2}$ scenario (low release of $\mathrm{I}_{2}$ ). These trends are also true for an release during summer conditions. Regarding speciation, there is a mix of $\mathrm{CH}_{3} \mathrm{OOI}$ and other unknown organic compounds. It can be viewed on Fig. 2 (a), Fig. 4 (a), and Fig. 7 (a) that $\mathrm{CH}_{3} \mathrm{OOI}$ is obtained by the following reaction

$$
\mathrm{I}+\mathrm{CH}_{3} \mathrm{O}_{2} \rightarrow \mathrm{CH}_{3} \mathrm{OOI}
$$

The methylperoxy radicals arise from the atmospheric VOC oxidation. The interaction of iodine with VOC in the atmosphere is therefore not negligible especially during summer when iodinated organic products formed can reach $10 \%$ of the total iodine mass.

There is only one reaction consuming the $\mathrm{CH}_{3} \mathrm{OOI}$ species

$$
\mathrm{I}+\mathrm{CH}_{3} \mathrm{OOI} \rightarrow \mathrm{I}_{2}+\mathrm{CH}_{3} \mathrm{O}_{2}
$$

The unknown organic compounds come from reactions experimentally observed without determination of all the products and the corresponding branching ratios (noted as "products" in the reaction mechanism). These species will be therefore accumulated. It is the case for example of the following reaction: 
$\mathrm{IO}+\mathrm{CH}_{3} \mathrm{O}_{2} \rightarrow$ products

The examination of literature data exhibits at least 10 different reaction channels (Bale et al., 2005; Dillon et al., 2006; Drougas and Kosmas, 2007; Enami et al., 2006)

$$
\begin{aligned}
& \mathrm{IO}+\mathrm{CH}_{3} \mathrm{O}_{2} \rightarrow \mathrm{CH}_{3} \mathrm{OI}+\mathrm{O}_{2} \\
& \mathrm{IO}+\mathrm{CH}_{3} \mathrm{O}_{2} \rightarrow \mathrm{CH}_{2} \mathrm{O}_{2}+\mathrm{HOI} \\
& \mathrm{IO}+\mathrm{CH}_{3} \mathrm{O}_{2} \rightarrow \mathrm{HCH}(\mathrm{O})+\mathrm{HOOI} \\
& \mathrm{IO}+\mathrm{CH}_{3} \mathrm{O}_{2} \rightarrow \mathrm{HCH}(\mathrm{O})+\mathrm{HOIO} \\
& \mathrm{IO}+\mathrm{CH}_{3} \mathrm{O}_{2} \rightarrow \mathrm{HCH}(\mathrm{O})+\mathrm{HI}+\mathrm{O}_{2} \\
& \mathrm{IO}+\mathrm{CH}_{3} \mathrm{O}_{2} \rightarrow \mathrm{HCH}(\mathrm{O})+\mathrm{HO}_{2}+\mathrm{I} \\
& \mathrm{IO}+\mathrm{CH}_{3} \mathrm{O}_{2} \rightarrow \mathrm{CH}_{3} \mathrm{O}+\mathrm{I}+\mathrm{O}_{2} \\
& \mathrm{IO}+\mathrm{CH}_{3} \mathrm{O}_{2} \rightarrow \mathrm{CH}_{3} \mathrm{O}+\mathrm{IOO} \\
& \mathrm{IO}+\mathrm{CH}_{3} \mathrm{O}_{2} \rightarrow \mathrm{CH}_{3} \mathrm{O}+\mathrm{OIO} \\
& \mathrm{IO}+\mathrm{CH}_{3} \mathrm{O}_{2} \rightarrow \mathrm{CH}_{3} \mathrm{I}+\mathrm{O}_{3}
\end{aligned}
$$

Previous modelling studies (Saiz-Lopez et al., 2016; Sommariva et al., 2012) only consider the formation of $\mathrm{I}, \mathrm{HO}_{2}$, and $\mathrm{HCH}(\mathrm{O})(\mathrm{R} 33$ path $\mathrm{VI})$. This biradicalar reaction is also complex to be treated from a theoretical point of view as it involves two different potential energy surfaces (singlet and triplet), the presence of different adducts $\left(\mathrm{CH}_{3} \mathrm{OOIO}, \mathrm{CH}_{3} \mathrm{OOOI}\right)$ and their atmospheric decomposition pathways.

\subsubsection{Organic iodine formation with $\mathrm{CH}_{3} \mathrm{I}$ release}

Fig. 9 presents the organic iodine evolution repartition with $\mathrm{CH}_{3} \mathrm{I}$ release at 7 am in January. $\mathrm{CH}_{3} \mathrm{I}$ represents $10 \%$ of organic iodine species after 13 hours for a low release compared to 15 hours for a medium release. Three other organic species are produced $\left(\mathrm{CH}_{2} \mathrm{IOH}, \mathrm{CH}_{2} \mathrm{IOO}\right.$, and $\mathrm{CH}_{3} \mathrm{OOI}$ ). Higher is the concentration release, higher is the $\mathrm{CH}_{2} \mathrm{IOH}$ production compared to $\mathrm{CH}_{2} \mathrm{IOO}$ and $\mathrm{CH}_{3} \mathrm{OOI}$. Similar trends are observed for release in summer and during night. The reaction mechanism leads to several intermediate organic iodine species before reaching the inorganic reaction scheme with the I atoms (see Fig. 2 (b)).

\subsection{Day/night cycle impact}

Previous analyses showed that iodine reactivity is totally different during day and night. Fig. 10 (a) and (b) presents a reactive scheme for an $I_{2}$ release during day and night in summer. 
During daytime, main reactions are driven by photolysis. $I_{2}$ is mainly dissociated by photolysis to form I radical

$\mathrm{I}_{2}+\mathrm{h} v \rightarrow \mathrm{I}+\mathrm{I}$

Photolysis reaction has also an impact on the iodine nitrate destruction

$\mathrm{IONO}_{2}+\mathrm{h} v \rightarrow 0.5 \mathrm{I}+0.5 \mathrm{NO}_{3}+0.5 \mathrm{IO}+0.5 \mathrm{NO}_{2}$

During the night, the iodine reactivity is driven by the nitrate radical. Its chemistry with iodinated species is not well known nowadays. The main reaction leads to the $\mathrm{IONO}_{2}$ formation

$\mathrm{I}_{2}+\mathrm{NO}_{3} \rightarrow \mathrm{I}+\mathrm{IONO}_{2}$

\subsection{Influence of the added reactions to the iodine mechanism}

A comparison was made with the most recent mechanism proposed by Saiz-Lopez and coworkers (Saiz-Lopez et al., 2016) (see Table 1) for two scenarios simulations (SDM-I ${ }_{2}$ and WDM- $\mathrm{I}_{2}$ ). The results are shown in the figures 11-12. The I_INORG. family (mostly $\mathrm{I}_{2}$ ) is more rapidly consumed with our mechanism. As a consequence, we observe a more important $\mathrm{I}_{\mathrm{x}} \mathrm{O}_{\mathrm{y}}$ formation (especially for the $\mathrm{SDM}-\mathrm{I}_{2}$ scenario). This behaviour can be attributed by the fact that in our mechanism we consider all $\mathrm{I}_{\mathrm{x}} \mathrm{O}_{\mathrm{y}}$ nucleation reactions as well as the chain reactions of $\mathrm{I}_{2} \mathrm{O}_{3}$ and $\mathrm{I}_{2} \mathrm{O}_{4}$ with $\mathrm{O}_{3}$ leading to the $\mathrm{I}_{2} \mathrm{O}_{5}$ formation.

\section{Conclusion}

A new gas-phase iodine mechanism containing 248 reactions combined to the RACM air quality mechanism was implemented into the ASTEC OD model in order to evaluate the behaviour of iodine fission products in the atmosphere. Different simulation scenarios carried out highlighted that $\mathrm{I}_{2}$ and $\mathrm{CH}_{3} \mathrm{I}$ can react with ambient pollutants like $\mathrm{O}_{3}, \mathrm{NO}_{\mathrm{x}}$, or VOC to form iodine nitroxides, iodine oxides, and other organic and inorganic iodinated species. The $\mathrm{I}_{2}$ reactivity is faster than that of $\mathrm{CH}_{3} \mathrm{I}$ and impacts the formation of other species.

Iodine nitroxides are mainly formed during night with low and medium releases in winter. Iodine nitrate is the main iodine nitroxide formed during night while $\mathrm{INO}_{2}$ can be formed during day especially if the $\mathrm{I}_{2}$ concentration increases.

Iodine oxides are promoted with a high release of $I_{2}$ during summer. This is due to a strong relationship between iodine oxides formation and ozone concentrations. In this work, $\mathrm{I}_{2} \mathrm{O}$, $\mathrm{I}_{2} \mathrm{O}_{5}$, and $\mathrm{HOIO}_{2}$ are the most abundant iodine oxides because their atmospheric removal 
pathways in the gas-phase are not yet known. The formation of organic iodine compounds can be highlighted in the case of a low $\mathrm{I}_{2}$ release. The main species will be $\mathrm{CH}_{3} \mathrm{OOI}$, which is produced by the reaction between I atom and the methyl peroxy radical. In case of a $\mathrm{CH}_{3} \mathrm{I}$ release, before producing the I atom, the reaction mechanism leads to either the formation or the destruction of several other organic iodinated compounds such as $\mathrm{CH}_{2} \mathrm{IOH}$ and $\mathrm{CH}_{2} \mathrm{IOO}$. Iodine organic reactivity should be improved to get firm conclusions.

\section{Acknowledgements}

We appreciated the support from the LABEX CaPPA (Chemical and Physical Properties of the Atmosphere), which is funded by the French National Research Agency (ANR) through the PIA (Programme d'Investissement d'Avenir) under contract "ANR-11-LABX-0005-01" and also the Regional Council "Hauts-de-France" and the "European Funds for Regional Economic Development". We thank anonymous reviewers for fruitful comments. 


\section{Table 1}

Gas-phase iodine mechanisms used in atmospheric modelling

\begin{tabular}{|c|c|c|c|c|c|c|}
\hline \# reactions & $\begin{array}{l}\text { \# Organic } \\
\text { reactions }\end{array}$ & $\begin{array}{l}\text { \# Inorganic } \\
\text { reactions }\end{array}$ & $\begin{array}{c}\text { \# Organic photolysis } \\
\text { reactions }\end{array}$ & $\begin{array}{l}\text { \# Inorganic photolysis } \\
\text { reactions }\end{array}$ & Model & Reference \\
\hline 23 & $1^{\mathrm{a}}$ & 13 & 3 & 6 & 0D & (McFiggans et al., 2000) \\
\hline 39 & $3^{\mathrm{a}, \mathrm{b}, \mathrm{c}}$ & 22 & 6 & 8 & 1D & (Pechtl et al., 2006) \\
\hline 36 & $1^{\mathrm{a}}$ & 27 & 3 & 5 & $1 \mathrm{D}$ & (Saiz-Lopez et al., 2008) \\
\hline 41 & $1^{\mathrm{a}}$ & 30 & 0 & 10 & $1 \mathrm{D}$ & (Mahajan et al., 2009) \\
\hline 45 & $1^{\mathrm{a}}$ & 33 & 3 & 8 & $0 \mathrm{D}$ & (Furneaux et al., 2010) \\
\hline 42 & $1^{\mathrm{a}}$ & 27 & 4 & 10 & $3 \mathrm{D}$ & (Ordóñez et al., 2012) \\
\hline 58 & $1^{\mathrm{d}}$ & 47 & 0 & 10 & $1 \mathrm{D}$ & (Sommariva et al., 2012) \\
\hline 59 & $3^{\mathrm{b}, \mathrm{c}, \mathrm{d}}$ & 40 & 4 & 12 & $1 \mathrm{D} / 3 \mathrm{D}$ & (Saiz-Lopez et al., 2016) \\
\hline 43 & $1^{\mathrm{a}}$ & 28 & 4 & 10 & $3 \mathrm{D}$ & (Sherwen et al., 2016a; Sherwen et al., 2016b) \\
\hline
\end{tabular}

${ }^{\mathrm{a}} \mathrm{IO}+\mathrm{CH}_{3} \mathrm{SCH}_{3 .}{ }^{\mathrm{b}} \mathrm{OH}+\mathrm{CH}_{3} \mathrm{I} .{ }^{\mathrm{c}} \mathrm{OH}+\mathrm{C}_{3} \mathrm{H}_{7} \mathrm{I} .{ }^{\mathrm{d}} \mathrm{IO}+\mathrm{CH}_{3} \mathrm{O}_{2}$. 
Table 2

IRACM organic class and organic iodine species associated

\begin{tabular}{|c|c|}
\hline IRACM class & Species \\
\hline I_ALD & $\mathrm{CH}_{3} \mathrm{C}(\mathrm{O}) \mathrm{I}$ \\
\hline I_CH4 & $\mathrm{CH}_{3} \mathrm{I}$ \\
\hline I_CSL & iodobenzene \\
\hline I_DIEN & iodocyclopentadiene \\
\hline I_ETE & $\mathrm{CH}_{2}=\mathrm{CHI}$ \\
\hline I_ETH & $\mathrm{CH}_{3} \mathrm{CH}_{2} \mathrm{I}$ \\
\hline I_ETHR & $\mathrm{CH}_{2} \mathrm{CH}_{2} \mathrm{I}, \mathrm{CH}_{3} \mathrm{CHI}$ \\
\hline I_HC3 & $\begin{array}{l}\mathrm{CH}_{3} \mathrm{CHICH}_{2} \mathrm{CH}_{3}, \mathrm{CH}_{2} \mathrm{ICH}_{2} \mathrm{CH}_{3}, \quad \mathrm{t}-\mathrm{C}_{4} \mathrm{H}_{9} \mathrm{I}, \quad \mathrm{s}-\mathrm{C}_{4} \mathrm{H}_{9} \mathrm{I}, \quad \mathrm{n}-\mathrm{C}_{3} \mathrm{H}_{7} \mathrm{I}, \\
\mathrm{i}-\mathrm{C}_{3} \mathrm{H}_{7} \mathrm{I}, \mathrm{t}-\mathrm{C}_{3} \mathrm{H}_{7} \mathrm{I}, \mathrm{CH}_{3} \mathrm{CHICH}_{3} \text {, iodomethylcyclopropane, } \mathrm{CH}_{2} \mathrm{IOH}\end{array}$ \\
\hline I_HC3R & $\begin{array}{l}\mathrm{CH}_{2} \mathrm{CH}_{2} \mathrm{CH}_{2} \mathrm{I}, \quad \mathrm{CH}_{3} \mathrm{CH}_{2} \mathrm{CHI}, \quad \mathrm{CH}_{3} \mathrm{CHCH}_{2} \mathrm{I}, \quad \mathrm{CH}_{2} \mathrm{CHICH}_{3}, \\
\mathrm{CH}_{3} \mathrm{CICH}_{3}, \mathrm{CH}_{2} \mathrm{IO}\end{array}$ \\
\hline I_HC5 & Iodocyclopentane \\
\hline I_HCHO & $\mathrm{HC}(\mathrm{O}) \mathrm{I}$ \\
\hline I_MO2 & $\mathrm{CH}_{2} \mathrm{IO}_{2}$ \\
\hline I_MR & $\mathrm{CH}_{2} \mathrm{I}$ \\
\hline I_OLI & iodocyclopentene \\
\hline I_OLT & $\mathrm{CH}_{2}=\mathrm{CHCH}_{2} \mathrm{I}$ \\
\hline I_OP1 & $\mathrm{CH}_{3} \mathrm{OOI}$ \\
\hline I_TOL & iodomethylbenzene \\
\hline PROD & Products \\
\hline I2_CH4 & $\mathrm{CH}_{2} \mathrm{I}_{2}$ \\
\hline I2_ETE & $\mathrm{CHI}=\mathrm{CHI}$ \\
\hline I2_ETH & $\mathrm{CH}_{2} \mathrm{ICH}_{2} \mathrm{I}, \mathrm{CH}_{3} \mathrm{CHI}_{2}$ \\
\hline I2_HC3 & $\mathrm{ICH}_{2} \mathrm{CH}_{2} \mathrm{CH}_{2} \mathrm{I}$ \\
\hline I2_MR & $\mathrm{CHI}_{2}$ \\
\hline I3_CH4 & $\mathrm{CHI}_{3}$ \\
\hline
\end{tabular}

Table 3

Initial concentration in the box model

\begin{tabular}{lcl}
\hline Species & $\begin{array}{c}\text { Concentration } \\
(\text { molecules cm }\end{array}$ & Reference \\
\hline $\mathrm{H}_{2}$ & $1.34 \times 10^{13}$ & (Jacob, 1999) \\
$\mathrm{H}_{2} \mathrm{O}$ & $3.16 \times 10^{17}$ & (Jacob, 1999) \\
$\mathrm{H}_{2} \mathrm{O}_{2}$ & $7.29 \times 10^{9}$ & (Acker et al., 2008) \\
$\mathrm{OH}$ & $5.59 \times 10^{6}$ & (Stone et al., 2012) \\
$\mathrm{HO}_{2}$ & $5.59 \times 10^{8}$ & (Stone et al., 2012) \\
$\mathrm{N}_{2}$ & $1.95 \times 10^{19}$ & (Jacob, 1999) \\
$\mathrm{O}_{2}$ & $4.87 \times 10^{18}$ & (Jacob, 1999) \\
\hline
\end{tabular}




\section{Table 4}

Emissions flux for NOx and VOC

\begin{tabular}{lc}
\hline & Emissions flux (molecule $\mathrm{cm}^{-3} \mathrm{~s}^{-1}$ ) \\
\hline $\mathrm{NO}_{\mathrm{x}}$ & $1.1 \times 10^{6}$ \\
VOC & $3.0 \times 10^{6}$ \\
\hline
\end{tabular}

\section{Table 5}

VOC speciation for the RACM mechanism

\begin{tabular}{lc}
\hline RACM species & Speciation $\%$ \\
\hline ALD & 0.53 \\
ORA2 & 1.54 \\
ETE & 6.71 \\
ETH & 3.53 \\
HC3 & 24.93 \\
HC5 & 23.34 \\
HC8 & 7.60 \\
HCHO & 2.04 \\
KET & 6.49 \\
OLI & 2.87 \\
OLT & 3.11 \\
TOL & 7.90 \\
XYL & 9.41 \\
\hline
\end{tabular}

\section{Table 6}

Model scenarios

\begin{tabular}{|c|c|c|c|c|c|c|c|c|c|c|c|c|}
\hline \multirow[t]{4}{*}{ Parameter } & \multicolumn{6}{|c|}{ Summer } & \multicolumn{6}{|c|}{ Winter } \\
\hline & \multicolumn{3}{|c|}{ Day-time scenarios } & \multicolumn{3}{|c|}{ Night-time Scenarios } & \multicolumn{3}{|c|}{ Day-time scenarios } & \multicolumn{3}{|c|}{ Night-time Scenarios } \\
\hline & $\begin{array}{l}\text { Low } \\
\text { iodine }\end{array}$ & $\begin{array}{c}\text { Medium } \\
\text { iodine }\end{array}$ & $\begin{array}{l}\text { High } \\
\text { iodine }\end{array}$ & $\begin{array}{l}\text { Low } \\
\text { iodine }\end{array}$ & $\begin{array}{l}\text { Medium } \\
\text { iodine }\end{array}$ & $\begin{array}{l}\text { High } \\
\text { iodine }\end{array}$ & $\begin{array}{l}\text { Low } \\
\text { iodine }\end{array}$ & $\begin{array}{l}\text { Medium } \\
\text { iodine }\end{array}$ & $\begin{array}{l}\text { High } \\
\text { iodine }\end{array}$ & $\begin{array}{l}\text { Low } \\
\text { iodine }\end{array}$ & $\begin{array}{l}\text { Medium } \\
\text { iodine }\end{array}$ & $\begin{array}{l}\text { High } \\
\text { iodine }\end{array}$ \\
\hline & SDL & SDM & $\mathrm{SDH}$ & SNL & SNM & $\mathrm{SNH}$ & WDL & WDM & WDH & WNL & WNM & WNH \\
\hline $\mathrm{I}_{2}(\mathrm{pptV})$ & 0.98 & 98 & 9800 & 0.98 & 98 & 9800 & 0.98 & 98 & 9800 & 0.98 & 98 & 9800 \\
\hline $\begin{array}{l}\mathrm{CH}_{3} \mathrm{I} \\
(\mathrm{pptV})\end{array}$ & 1.96 & 196 & 19600 & 1.96 & 196 & 19600 & 1.96 & 196 & 19600 & 1.96 & 196 & 19600 \\
\hline \multicolumn{13}{|l|}{ Release } \\
\hline $\begin{array}{l}\text { Time } \\
\text { (hour) }\end{array}$ & 4 & 4 & 4 & 21 & 21 & 21 & 7 & 7 & 7 & 18 & 18 & 18 \\
\hline
\end{tabular}




\section{Figures}

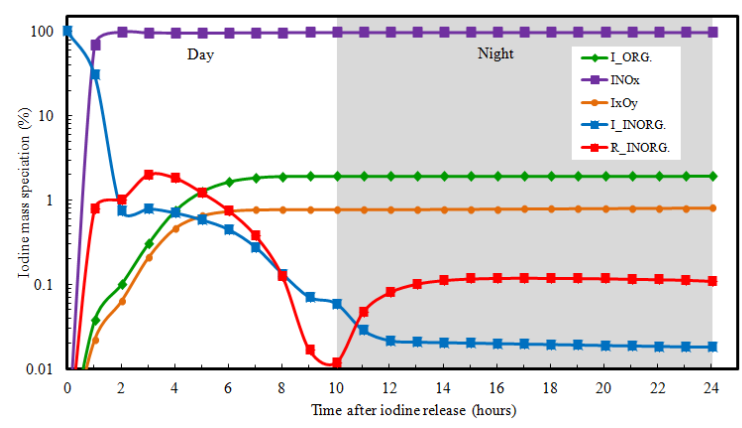

(a) $\mathrm{WDL}_{-} \mathrm{I}_{2}$

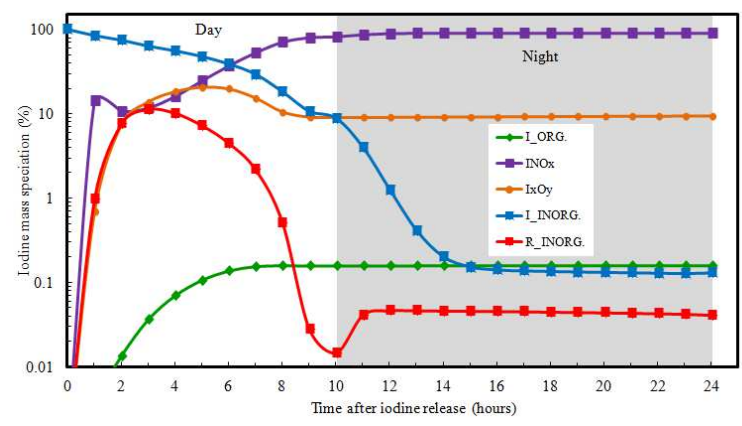

(c) $\mathrm{WDM}-\mathrm{I}_{2}$

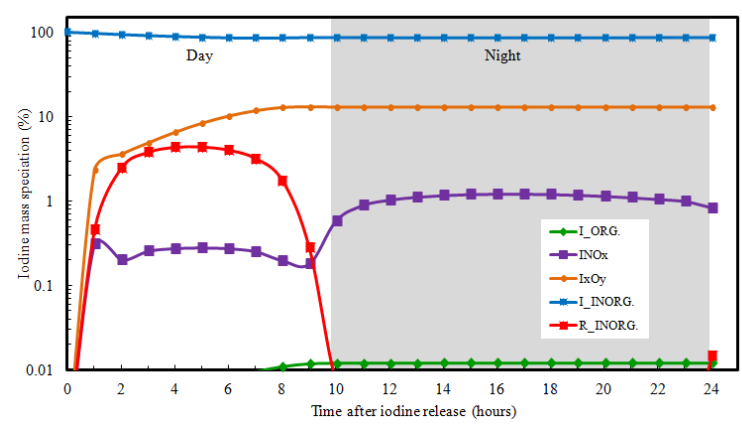

(e) $\mathrm{WDH}_{-} \mathrm{I}_{2}$

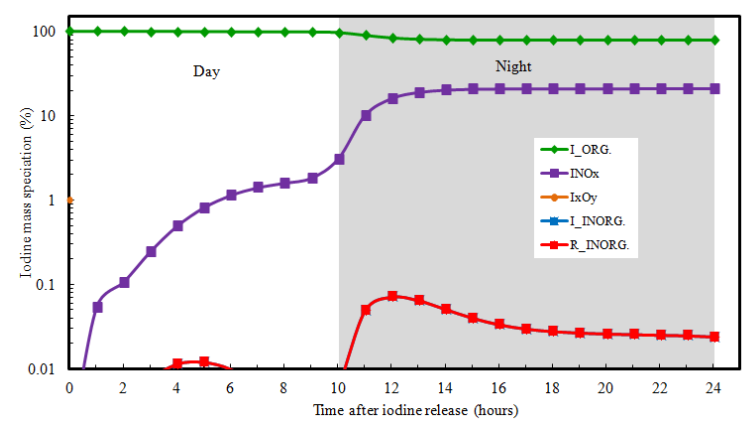

(b) $\mathrm{WDL}-\mathrm{CH}_{3} \mathrm{I}$

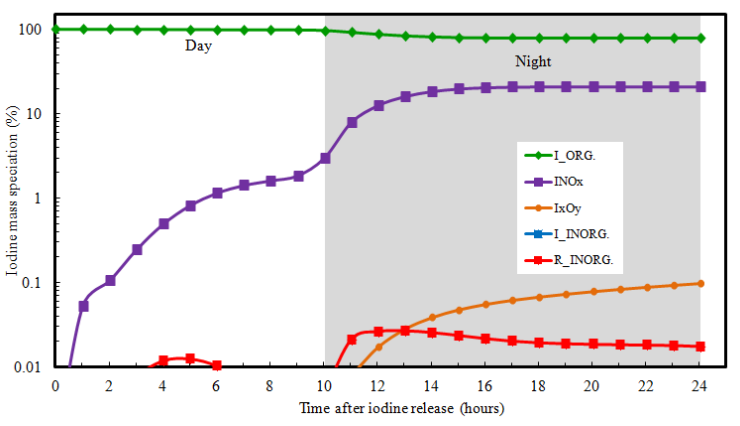

(d) $\mathrm{WDM}-\mathrm{CH}_{3} \mathrm{I}$

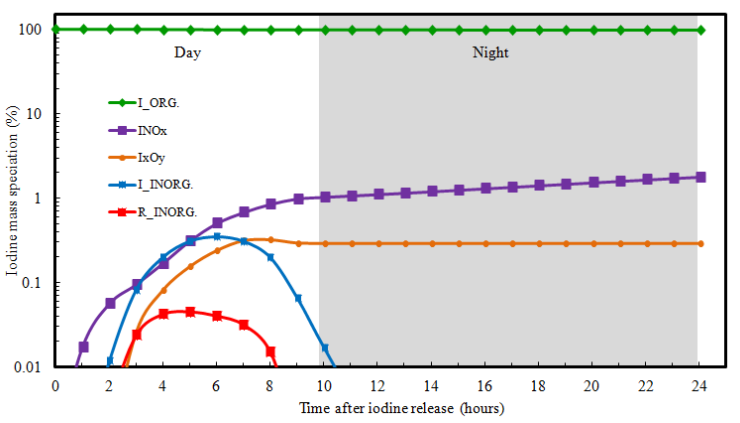

(f) $\mathrm{WDH}-\mathrm{CH}_{3} \mathrm{I}$

Fig. 1. Family mass evolution of iodine, 2013 January $1^{\text {st }}$, for an iodine release at 7 am with 0.98 pptV (a), 98 pptV (c), 9800 pptV (e) of $\mathrm{I}_{2}$ and 1.96 pptV (b), $196 \mathrm{pptV}$ (d), $19600 \mathrm{pptV}$ (f) of $\mathrm{CH}_{3} \mathrm{I}$. 


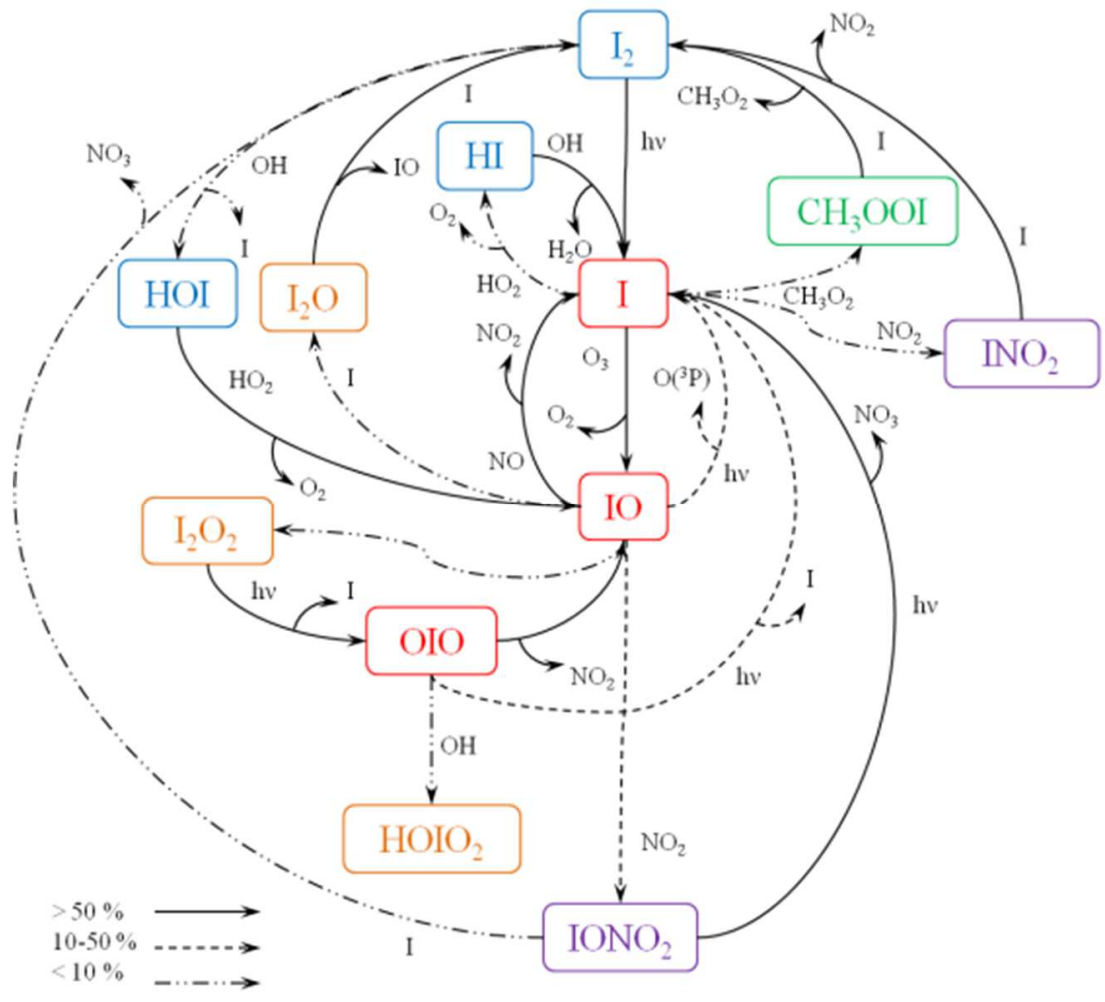

(a) WDL-I 2

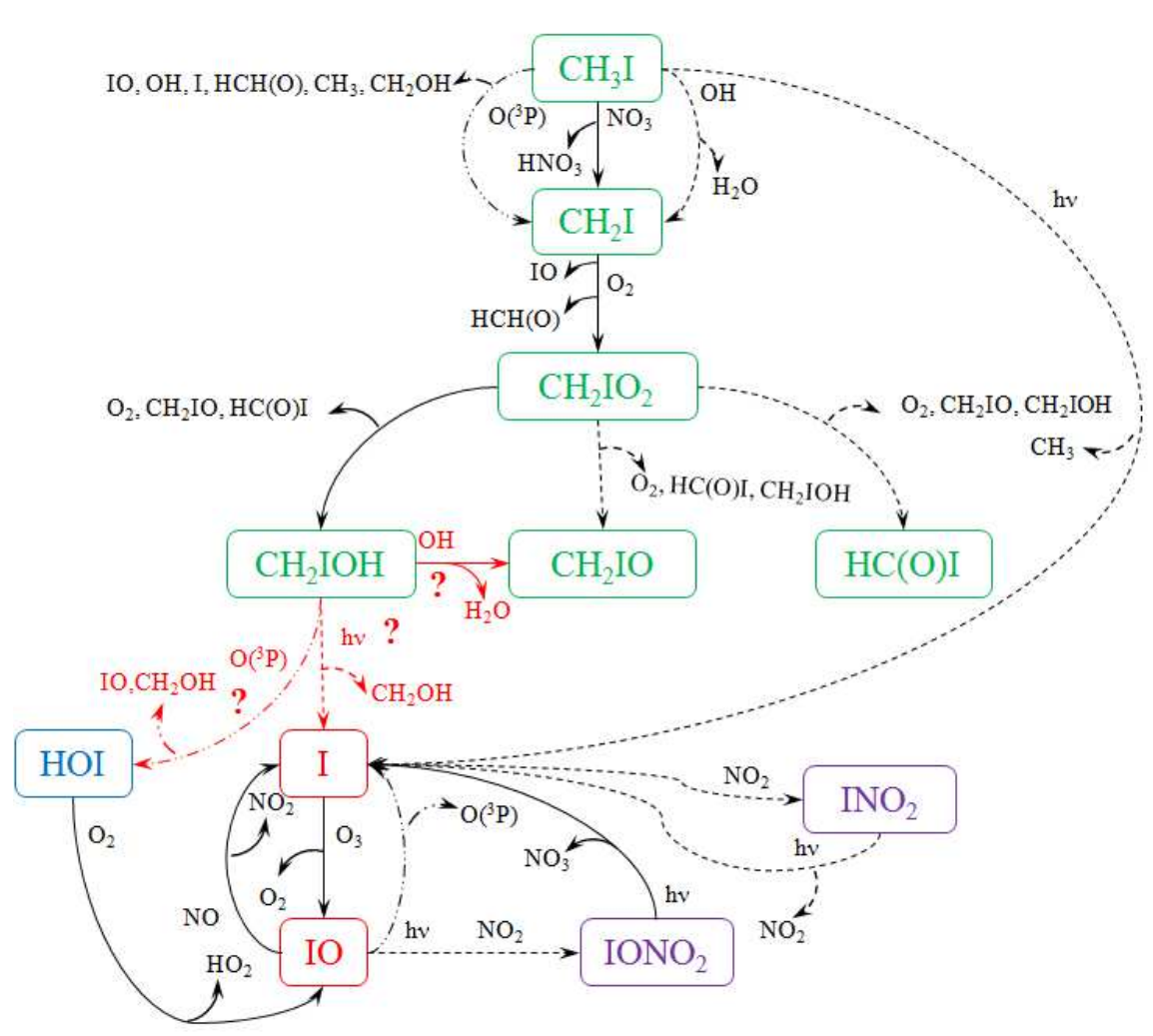

(b) $\mathrm{WDL}^{-\mathrm{CH}_{3} \mathrm{I}}$

Fig. 2. Iodine reaction scheme, 2013 January $1^{\text {st }}$ for iodine release at 7 am with $0.98 \mathrm{pptV}$ of $\mathrm{I}_{2}$ (a) and $1.96 \mathrm{pptV}$ of $\mathrm{CH}_{3} \mathrm{I}(\mathrm{b})$. 


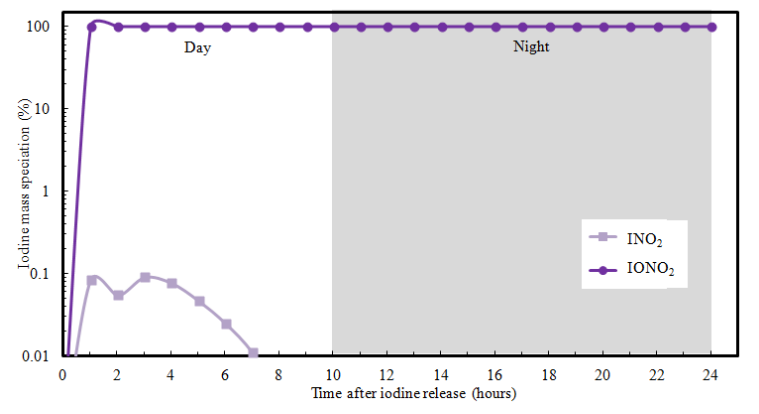

(a) WDL-I 2

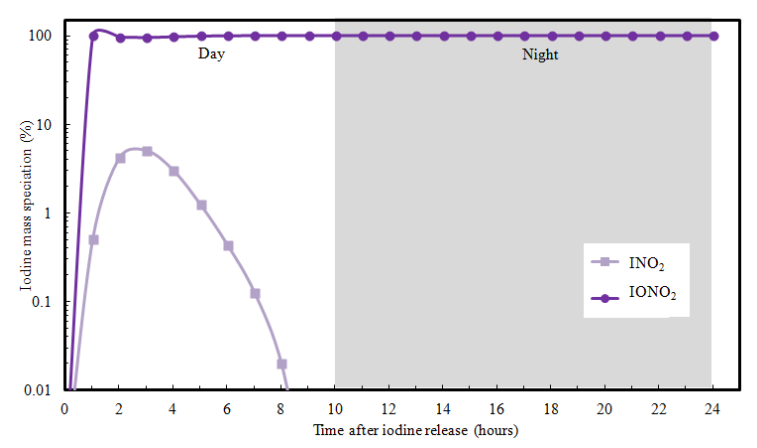

(c) $\mathrm{WDM}-\mathrm{I}_{2}$

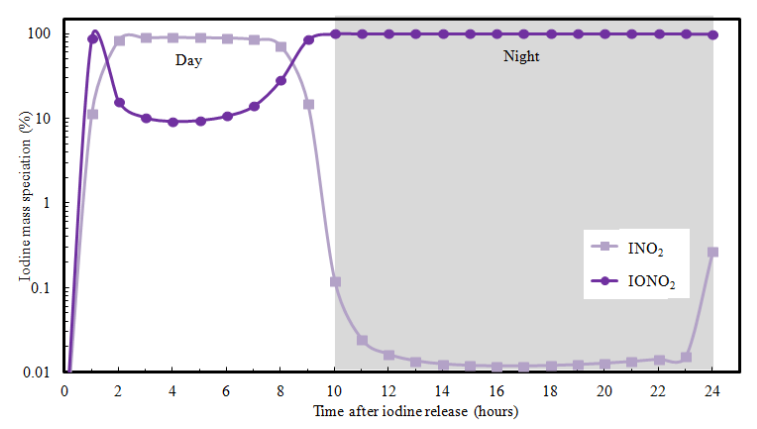

(e) $\mathrm{WDH}-\mathrm{I}_{2}$

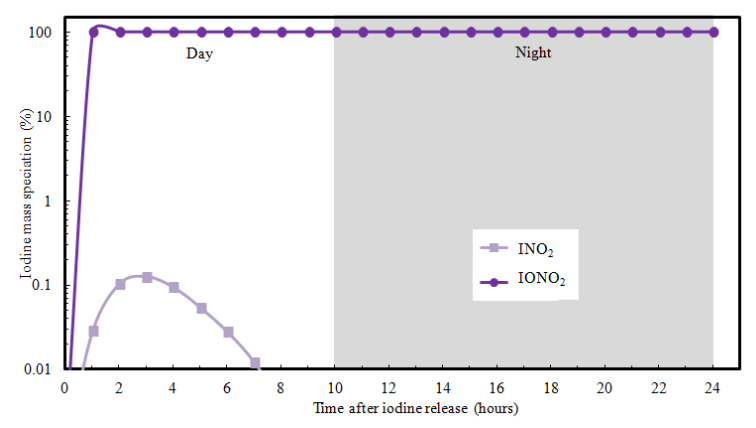

(b) $\mathrm{WDL}-\mathrm{CH}_{3} \mathrm{I}$

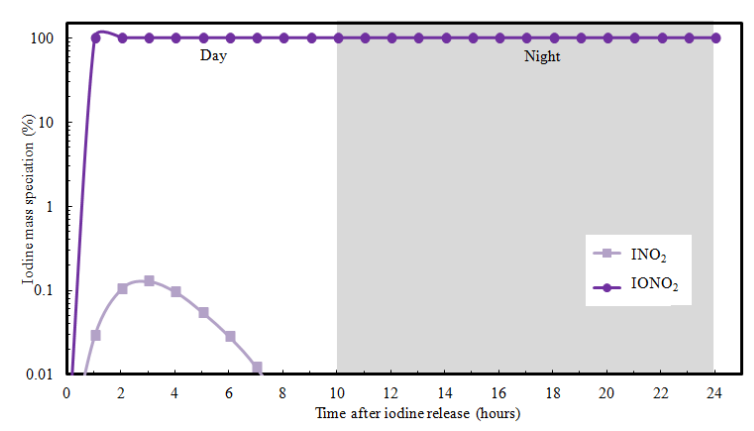

(d) $\mathrm{WDM}-\mathrm{CH}_{3} \mathrm{I}$

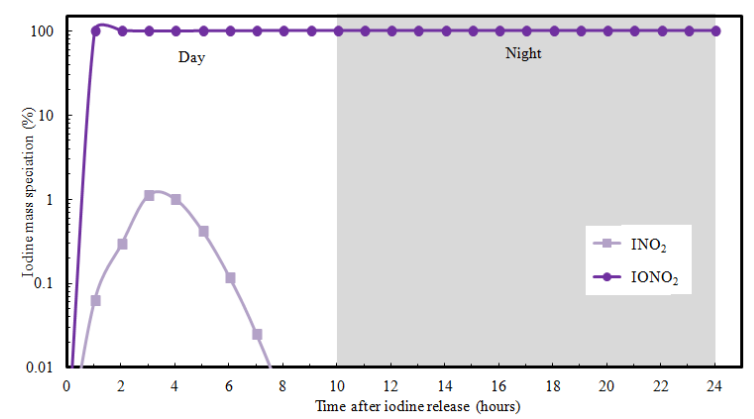

(f) $\mathrm{WDH}-\mathrm{CH}_{3} \mathrm{I}$

Fig. 3. Iodine nitroxides normalized mass speciation evolution, 2013 January $1^{\text {st }}$, for iodine release at 7 am with $0.98 \mathrm{pptV}$ (a), $98 \mathrm{pptV}$ (c), $9800 \mathrm{pptV}$ (e) of $\mathrm{I}_{2}$ and $1.96 \mathrm{pptV}$ (b), 196 pptV (d), 19600 pptV (f) of $\mathrm{CH}_{3} \mathrm{I}$. 


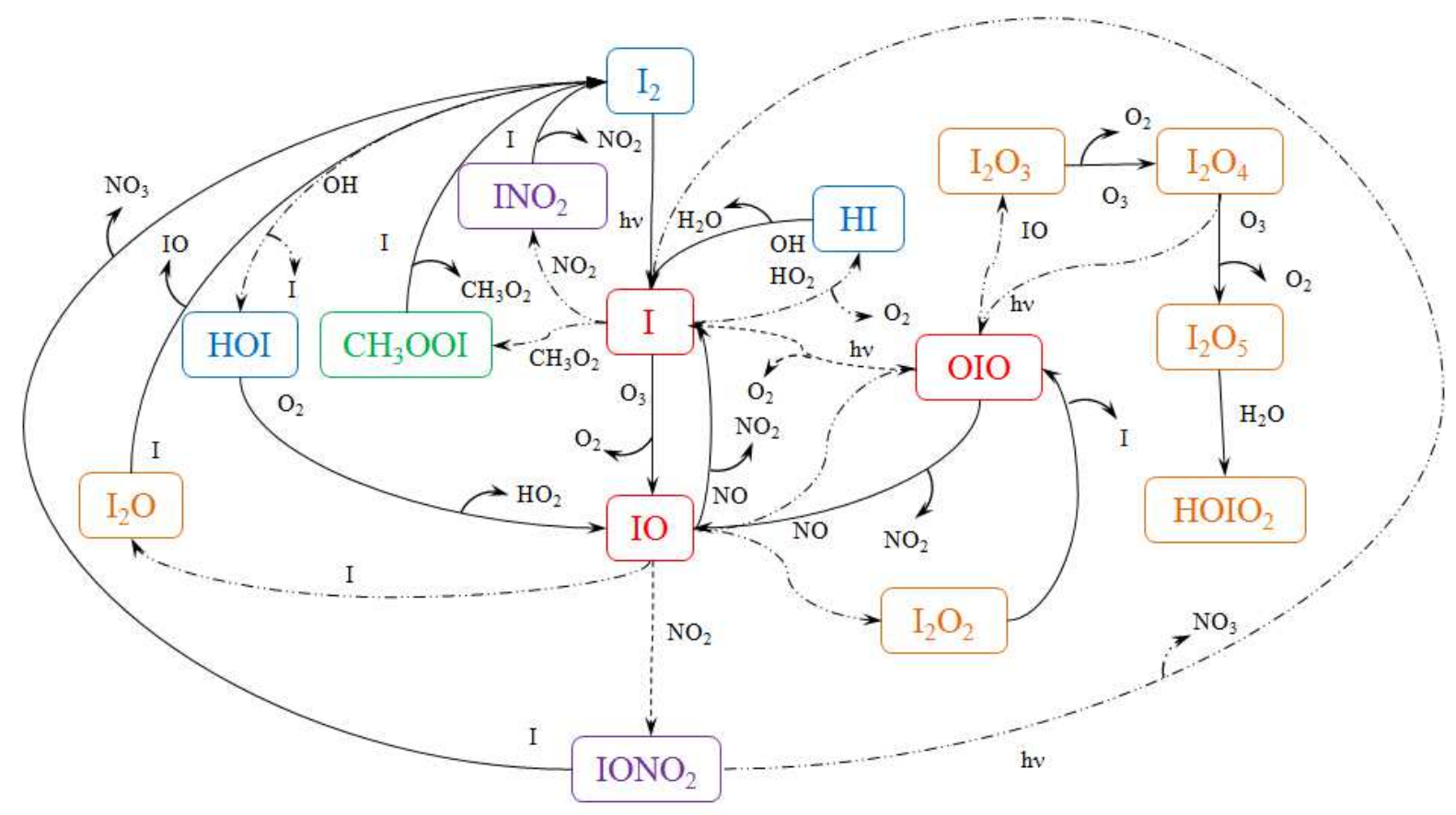

(a) WDM-I 2

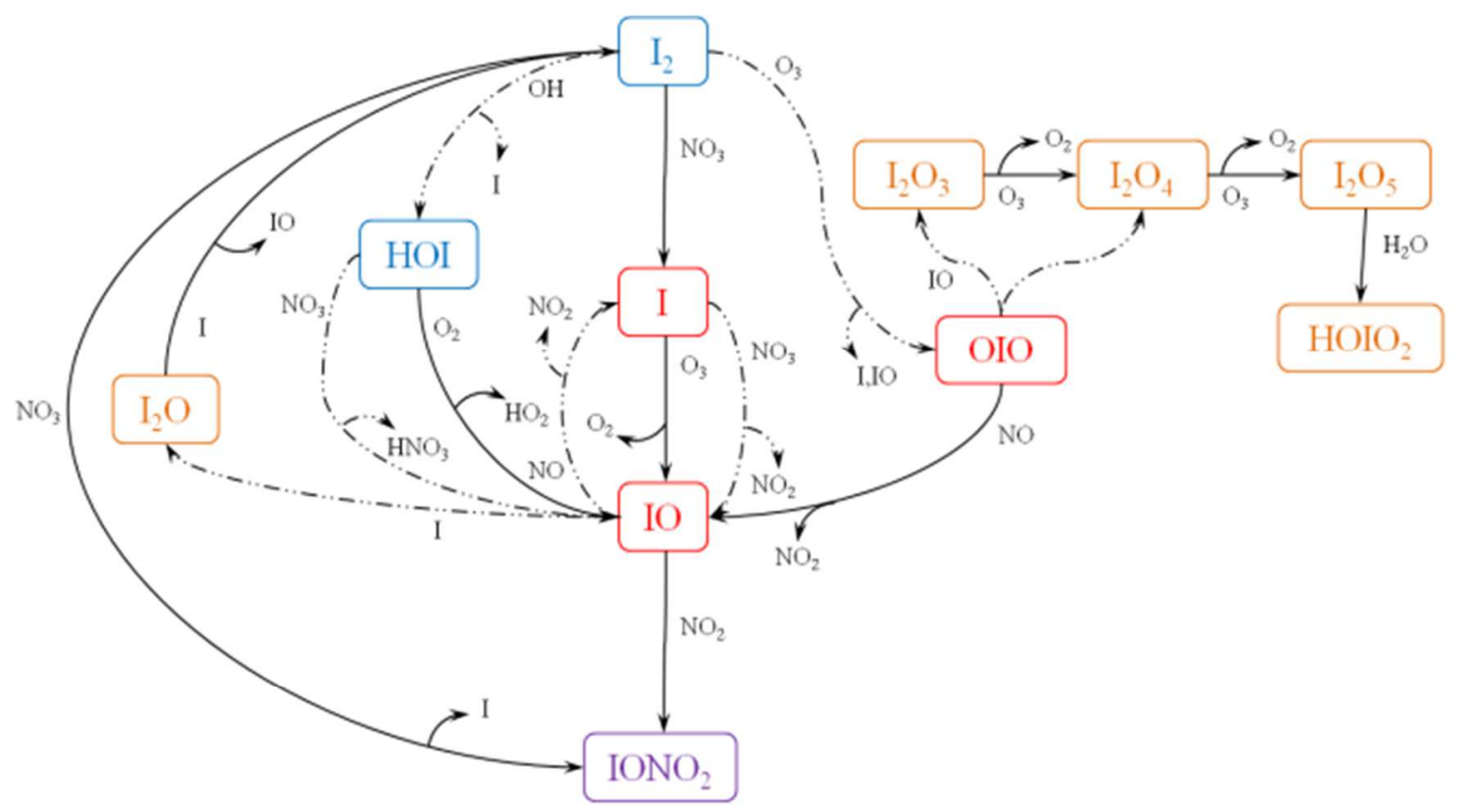

(b) WNM-I 2

Fig. 4. Iodine reaction scheme for $98 \mathrm{pptV}$ of $\mathrm{I}_{2}$ release, 2013 January $1^{\text {st }}$, at 7 am (a) and 6 pm (b). 


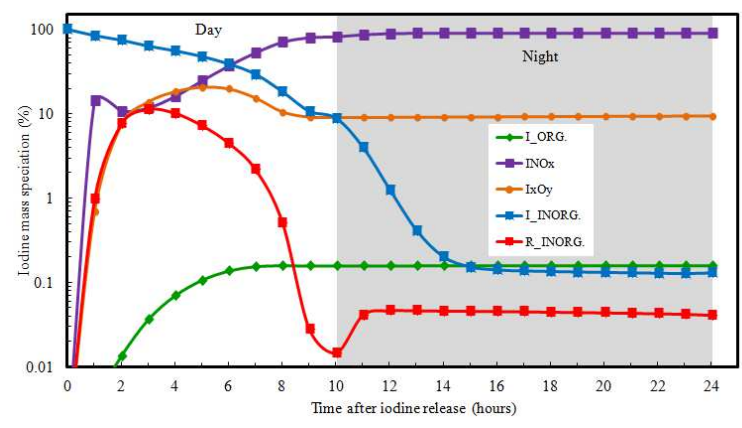

(a) $\mathrm{WDM}_{2} \mathrm{I}_{2}$

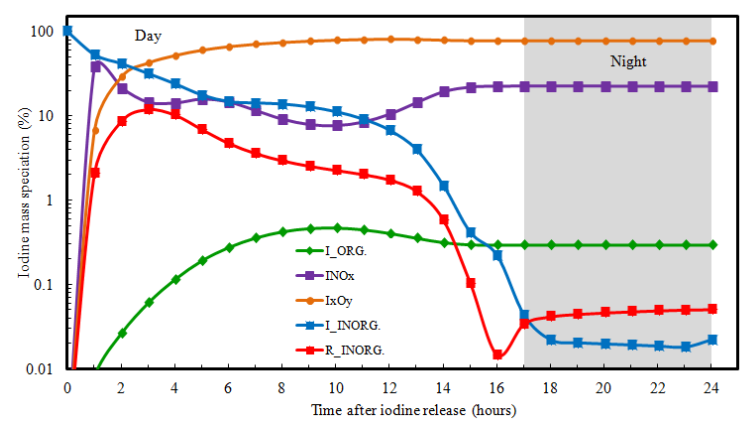

(c) $\mathrm{SDM}-\mathrm{I}_{2}$

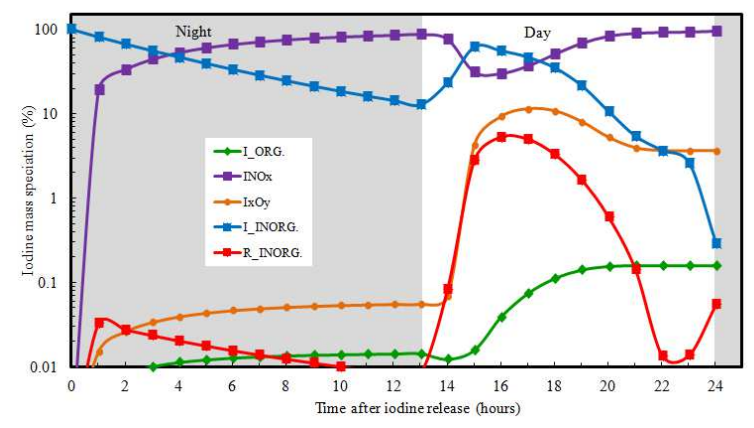

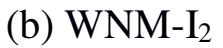

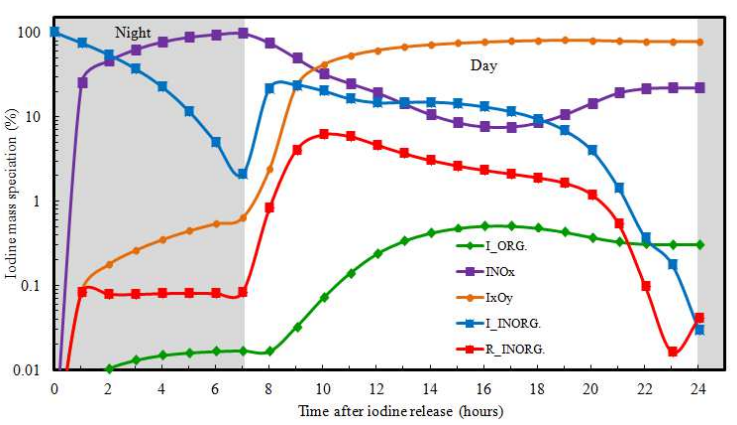

(d) $\mathrm{SNM}-\mathrm{I}_{2}$

Fig. 5. Family mass evolution of iodine, for an iodine release of $98 \mathrm{pptV}, 2013$ January $1^{\text {st }}$, at 7 am (a) and 6 pm (b), 2013 August $8^{\text {th }}$ at 4 am (c) and 9 pm (d).

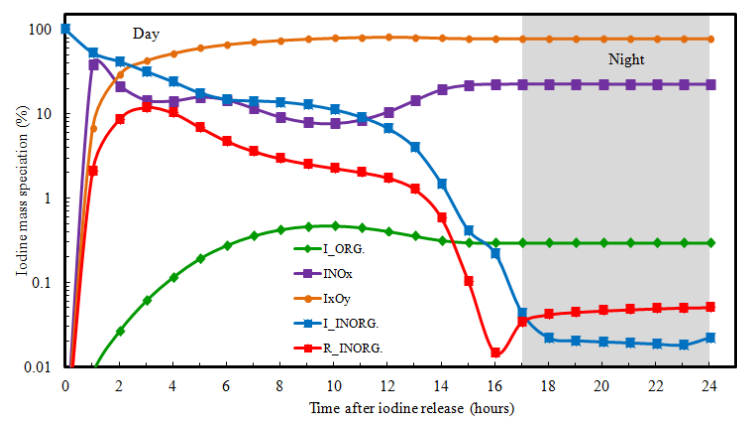

(a) $\mathrm{SDM}-\mathrm{I}_{2}$

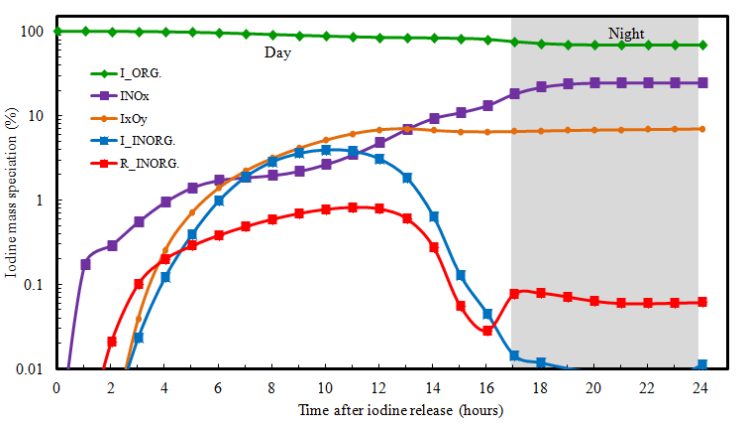

(b) $\mathrm{SDM}-\mathrm{CH}_{3} \mathrm{I}$

Fig. 6. Family mass evolution of iodine, 2013 August $8^{\text {th }}$, for an iodine release at 4 am with $98 \mathrm{pptV}$ of $\mathrm{I}_{2}$ (a) and $196 \mathrm{pptV}$ of $\mathrm{CH}_{3} \mathrm{I}(\mathrm{b})$. 


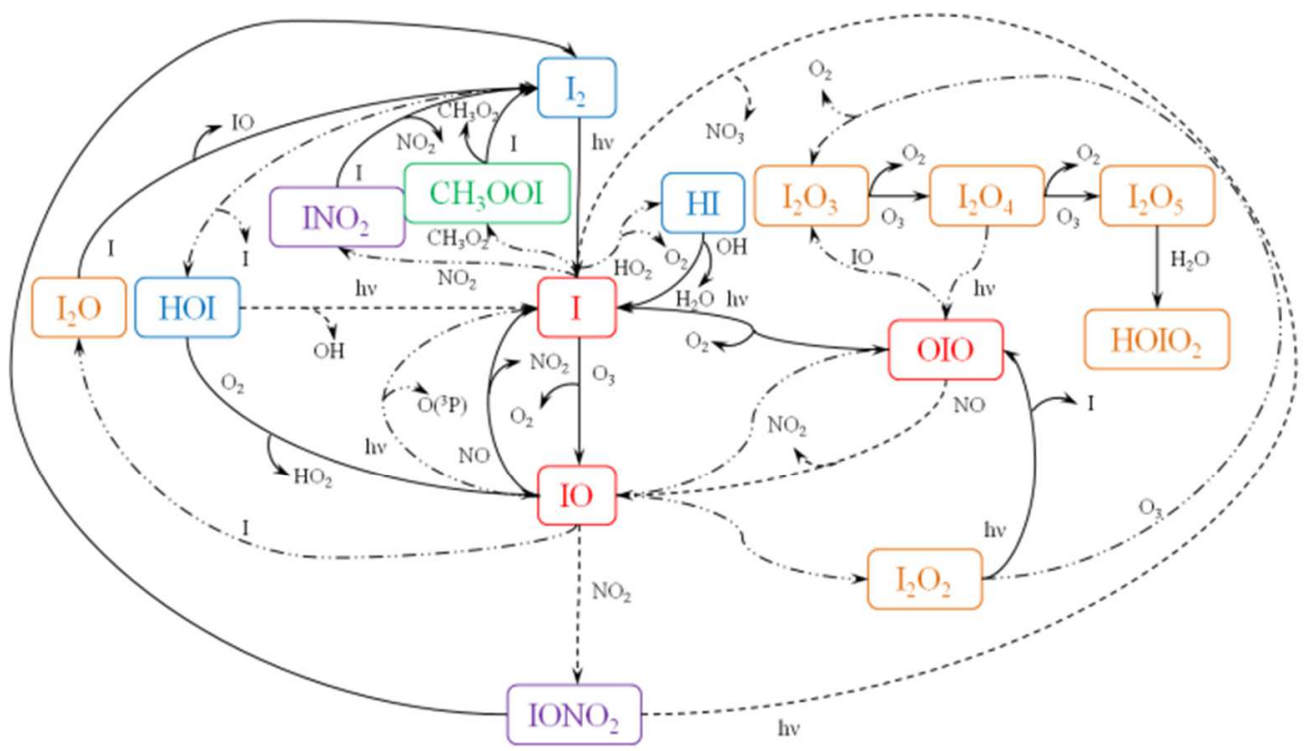

(a) $\mathrm{SDM}-\mathrm{I}_{2}$

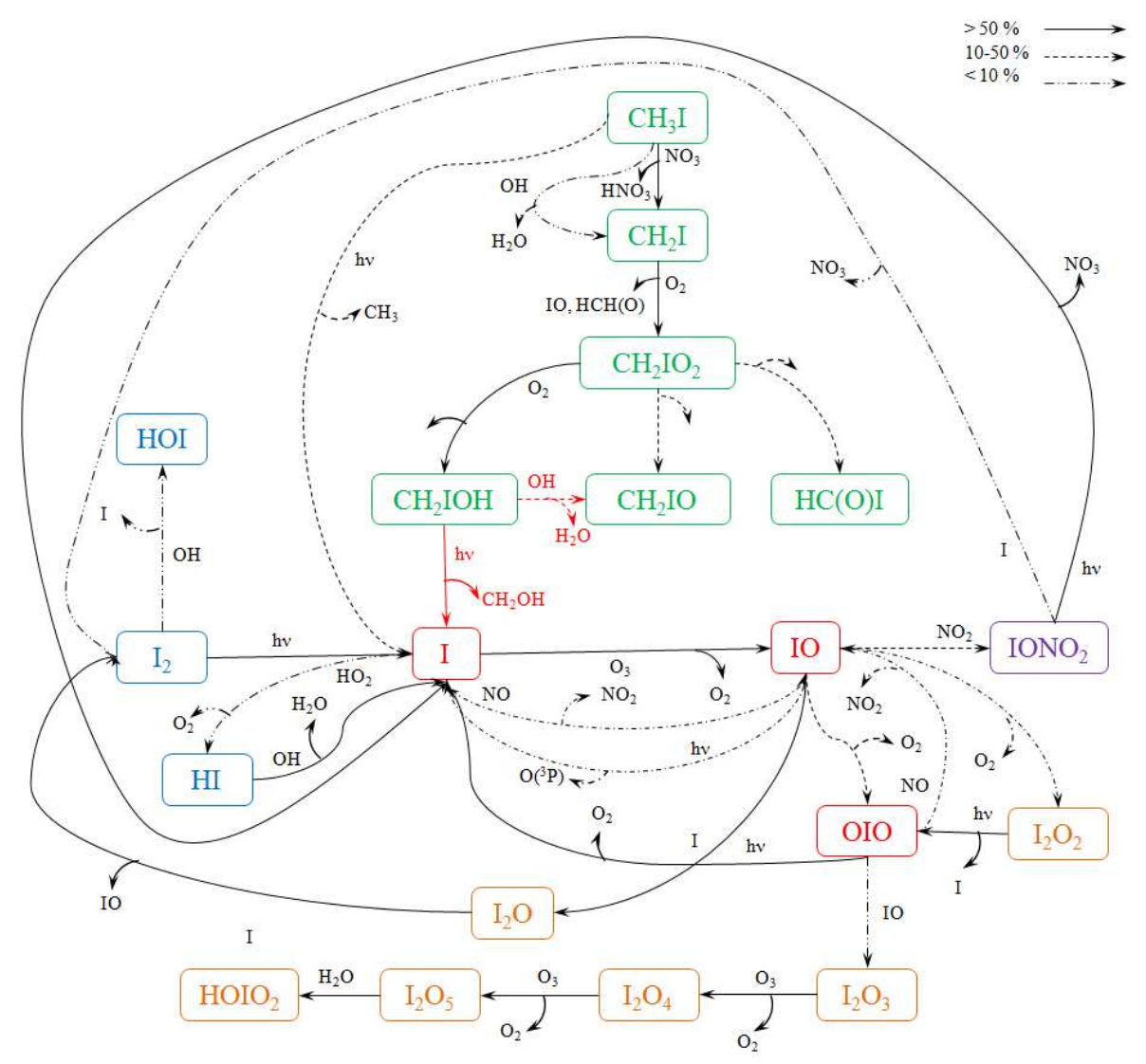

(b) $\mathrm{SDM}-\mathrm{CH}_{3} \mathrm{I}$

Fig. 7. Iodine reaction scheme, 2013 August $8^{\text {th }}$, for an iodine release at 4 am with $98 \mathrm{pptV}$ of $\mathrm{I}_{2}$ (a) and $196 \mathrm{pptV}$ of $\mathrm{CH}_{3} \mathrm{I}(\mathrm{b})$. 

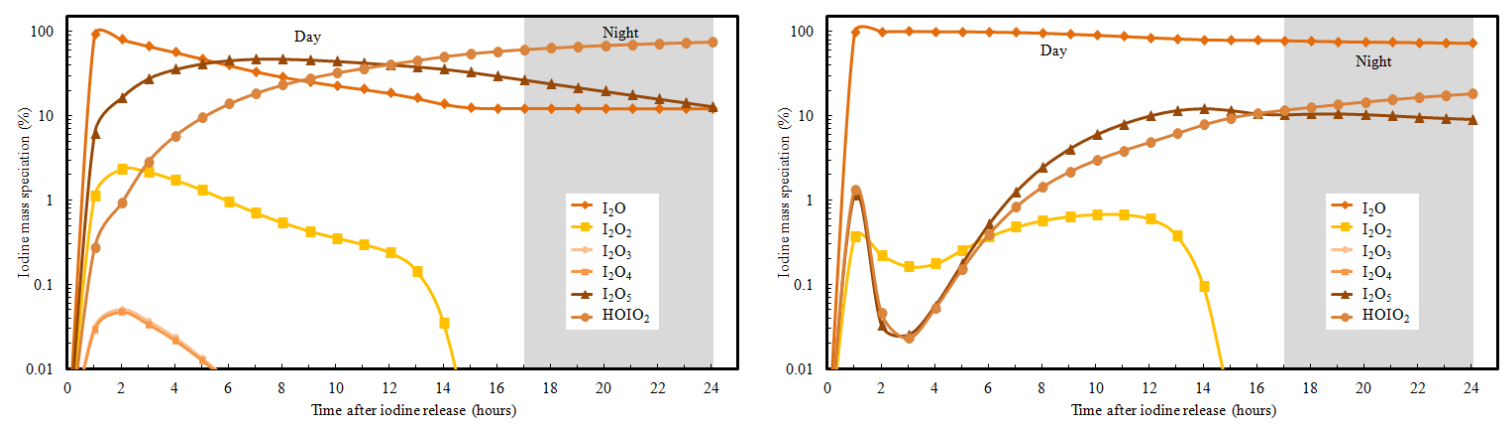

Fig. 8. Iodine oxide normalized mass speciation evolution, 2013 August $8^{\text {th }}$, for an iodine release at 4 am with 98 pptV of $\mathrm{I}_{2}$ (a) and $196 \mathrm{pptV}$ of $\mathrm{CH}_{3} \mathrm{I}$ (b).

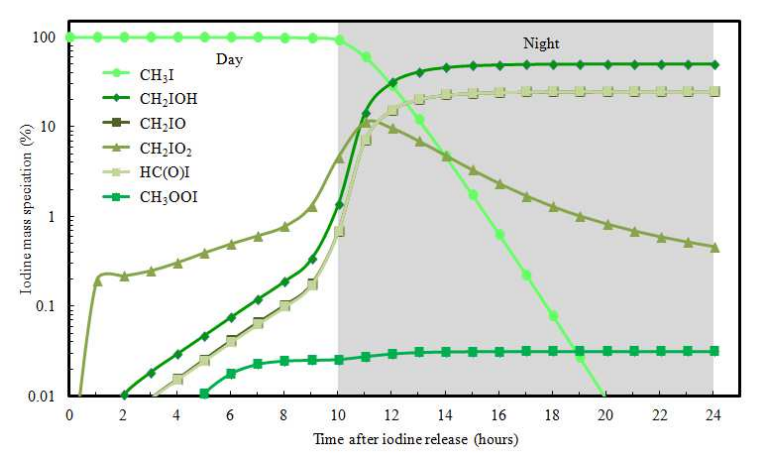

(a) $\mathrm{WDL}-\mathrm{CH}_{3} \mathrm{I}$

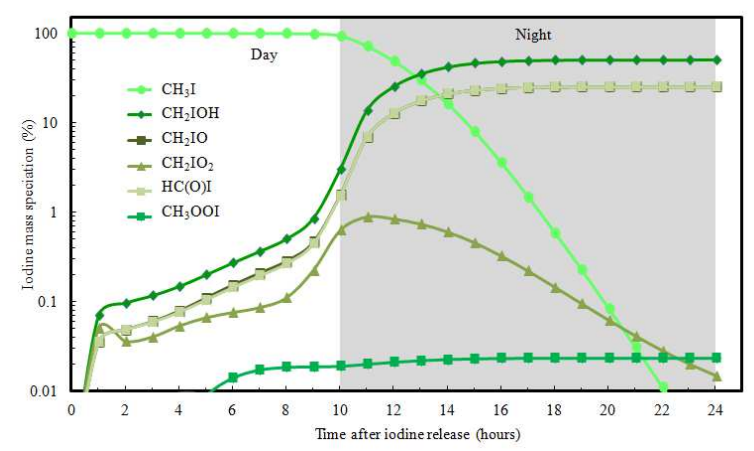

(b) $\mathrm{WDM}-\mathrm{CH}_{3} \mathrm{I}$

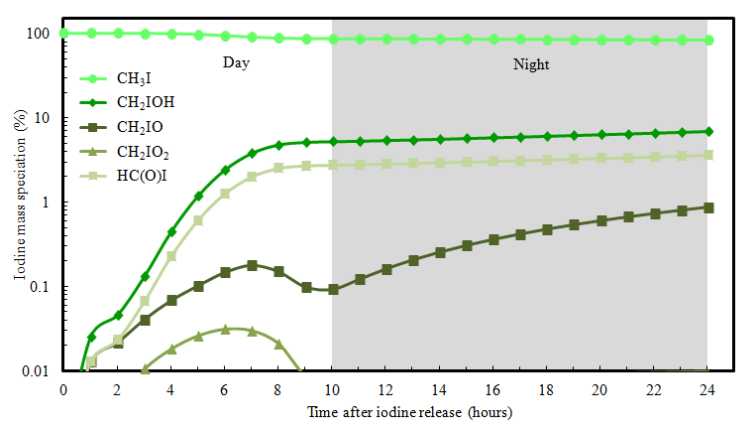

(c) $\mathrm{WDH}-\mathrm{CH}_{3} \mathrm{I}$

Fig. 9. Organic iodine normalized mass speciation evolution, 2013 January $1^{\text {st }}$, for iodine release at 7 am with $1.96 \mathrm{pptV}$ (a), $196 \mathrm{pptV}$ (b) and $19600 \mathrm{pptV}$ (c) of $\mathrm{CH}_{3} \mathrm{I}$. 


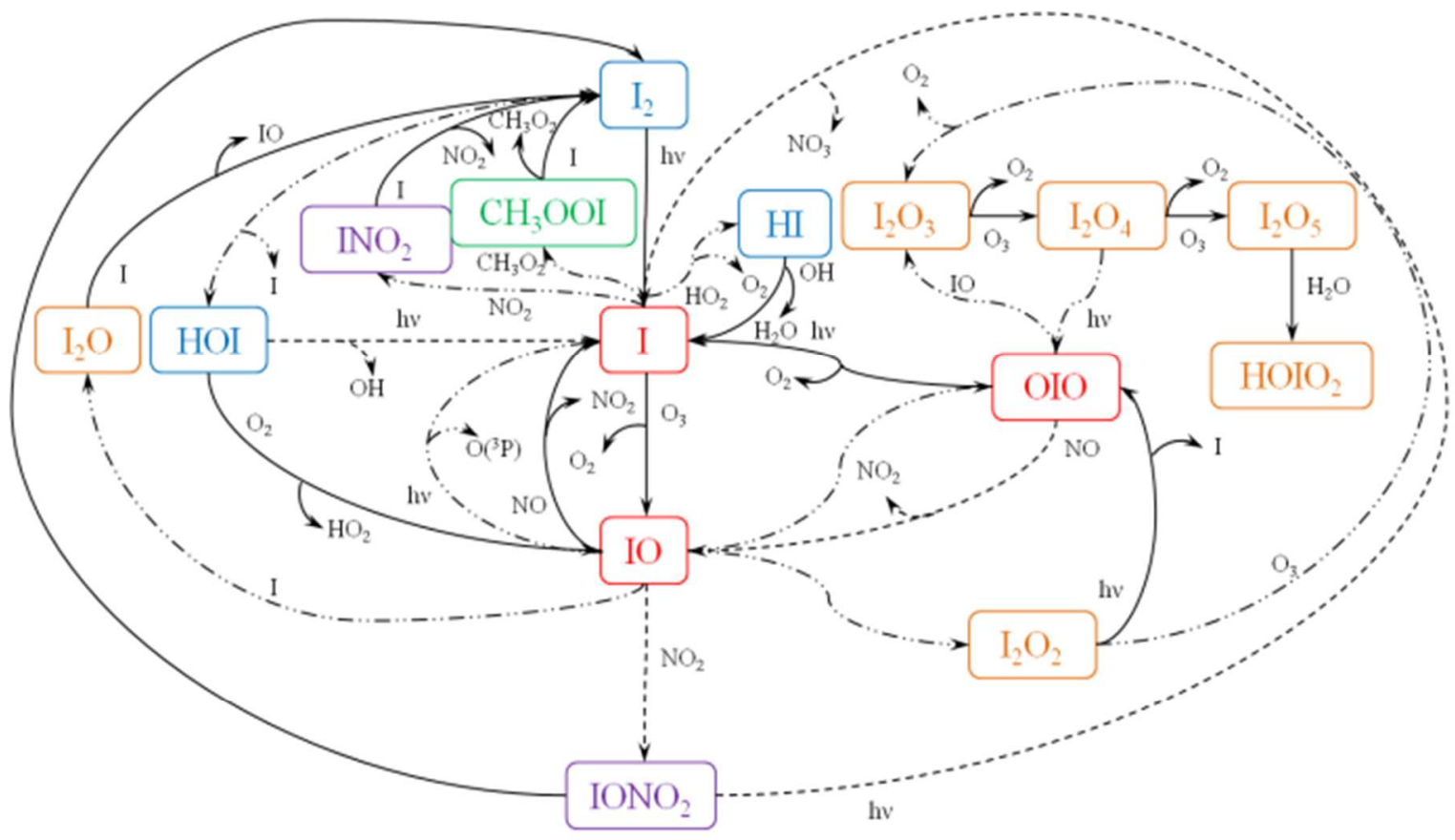

(a) $\mathrm{SDM}-\mathrm{I}_{2}$

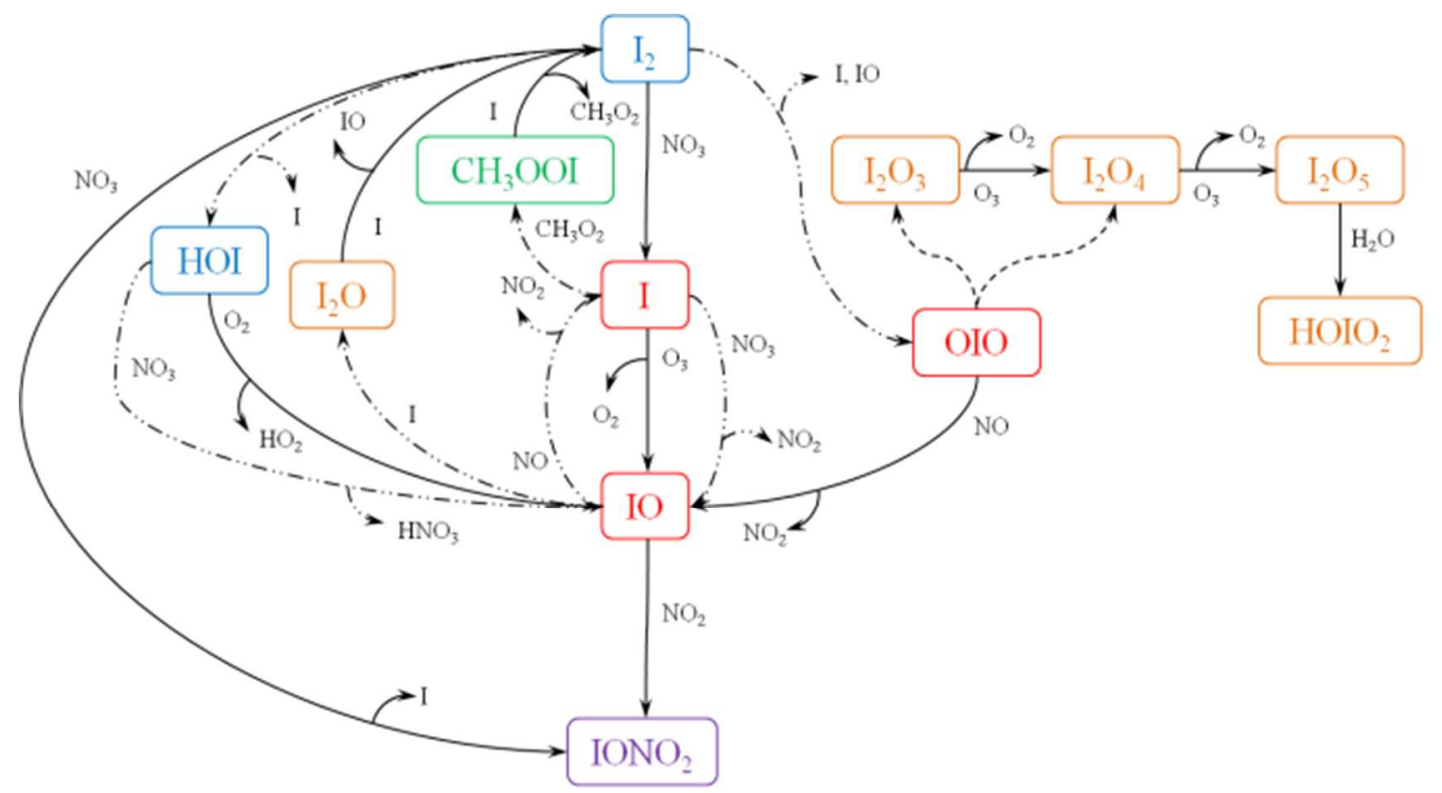

(b) $\mathrm{SNM}-\mathrm{I}_{2}$

Fig. 10. Iodine reaction scheme for $98 \mathrm{pptV}$ of $\mathrm{I}_{2}$ release, 2013 August $8^{\text {th }}$, at 4 am (a) and 9 $\mathrm{pm}(\mathrm{b})$. 


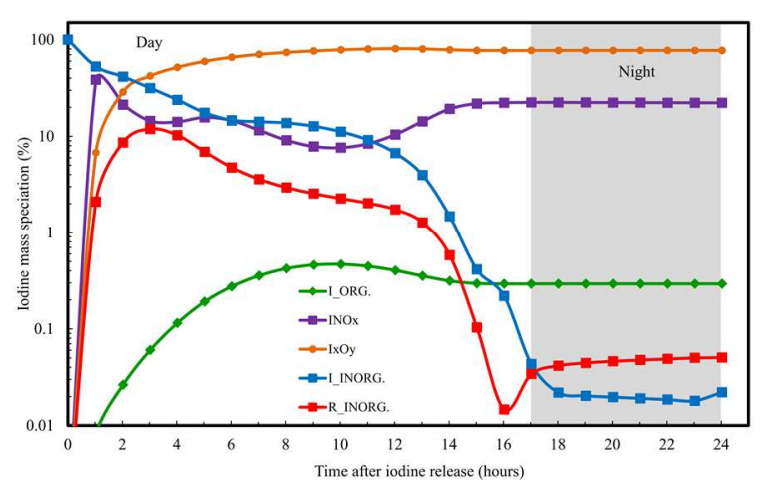

This work $\left(\mathrm{SDM}-\mathrm{I}_{2}\right)$

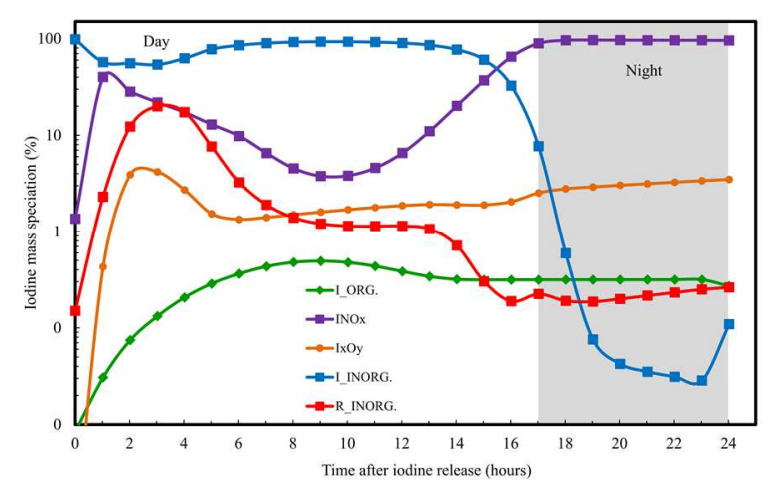

Saiz-Lopez et al. $2016\left(\mathrm{SDM}-\mathrm{I}_{2}\right)$

Fig. 11. Family mass evolution of iodine, for an iodine release of $98 \mathrm{pptV}, 2013$ August $8^{\text {th }}$ at $4 \mathrm{am}$.

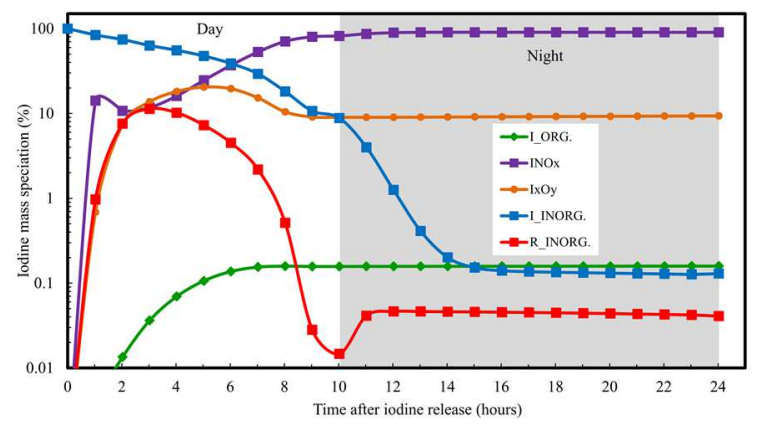

This work (WDM-I $\left.{ }_{2}\right)$

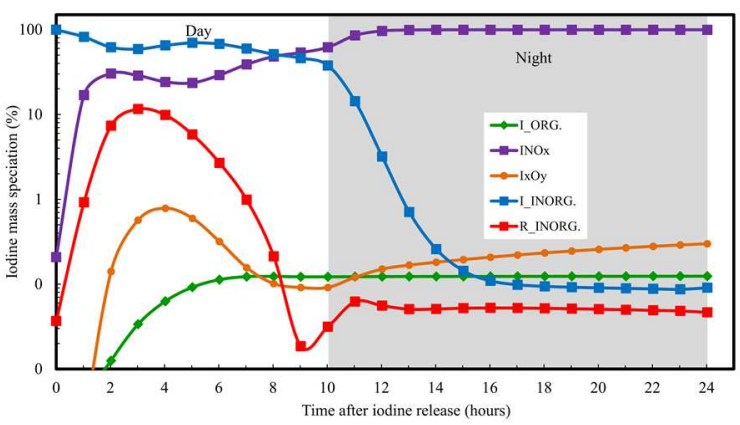

Saiz-Lopez et al. 2016 (WDM-I 2 )

Fig. 12. Family mass evolution of iodine, for an iodine release of $98 \mathrm{pptV}, 2013$ January $1^{\text {st }}$, at $7 \mathrm{am}$. 


\begin{tabular}{|c|c|c|c|c|}
\hline$\#$ & Reaction & $\begin{array}{c}\text { Rate coefficient } \\
\left(\mathrm{s}^{-1}\right) \text { or }\left(\mathrm{cm}^{3} \text { molecule } \mathrm{s}^{-1}\right) \\
\text { or }\left(\mathrm{cm}^{6} \text { molecule } \mathrm{s}^{-1}\right)\end{array}$ & Reference & IRACM reaction \\
\hline 1 & $\mathrm{HI}+\mathrm{CH}_{2}=\mathrm{CH}_{2} \rightarrow \mathrm{CH}_{3} \mathrm{CH}_{2} \mathrm{I}$ & $5.50 \times 10^{-16} \exp (-14560 / T)$ & (Benson and Bose, 1962) & $\mathrm{HI}+\mathrm{ETE} \rightarrow \mathrm{I}$ _ETH \\
\hline 2 & $\mathrm{HI}+\mathrm{CH}_{3} \mathrm{CH}=\mathrm{CHCH}_{3} \rightarrow \mathrm{CH}_{3} \mathrm{CHICH}_{2} \mathrm{CH}_{3}$ & $3.02 \times 10^{-15} \exp (-10605 / T)$ & (Nangia and Benson, 1964) & $\mathrm{HI}+\mathrm{OLI} \rightarrow \mathrm{I} \_\mathrm{HC} 3$ \\
\hline 3 & $\mathrm{HI}+$ cyclopentene $\rightarrow$ iodocyclopentane & $4.17 \times 10^{-13} \exp (-13477 / T)$ & (Furuyama et al., 1970a) & $\mathrm{HI}+\mathrm{OLI} \rightarrow \mathrm{I} \_\mathrm{HC} 5$ \\
\hline 4 & $\mathrm{HI}+\mathrm{CH}_{2}=\mathrm{CHCH}_{3} \rightarrow \mathrm{CH}_{2} \mathrm{ICH}_{2} \mathrm{CH}_{3}$ & $1.29 \times 10^{-13} \exp (-11805 / T)$ & (Bose and Benson, 1962) & $\mathrm{HI}+\mathrm{OLT} \rightarrow \mathrm{I} \_\mathrm{HC} 3$ \\
\hline 5 & $\mathrm{HI}+\mathrm{i}-\mathrm{C}_{4} \mathrm{H}_{8} \rightarrow \mathrm{t}-\mathrm{C}_{4} \mathrm{H}_{9} \mathrm{I}$ & $5.25 \times 10^{-15} \exp (-9061 / T)$ & (Bose and Benson, 1963) & $\mathrm{HI}+\mathrm{OLT} \rightarrow \mathrm{I} \_\mathrm{HC} 3$ \\
\hline 6 & $\mathrm{I}+$ benzaldehyde $\rightarrow \mathrm{HI}+$ benzoyl & $1.99 \times 10^{-11} \exp (-8399 / T)$ & (Solly and Benson, 1971) & $\mathrm{I}+\mathrm{ALD} \rightarrow \mathrm{HI}+\mathrm{ALDR}$ \\
\hline 7 & $\mathrm{I}+\mathrm{CH}_{2} \mathrm{CHCH}(\mathrm{O}) \rightarrow \mathrm{HI}+\mathrm{CH}_{2} \mathrm{CHCO}$ & $2.09 \times 10^{-11} \exp (-8459 / T)$ & (Alfassi and Golden, 1973b) & $\mathrm{I}+\mathrm{ALD} \rightarrow \mathrm{HI}+\mathrm{ALDR}$ \\
\hline 8 & $\mathrm{I}+\mathrm{CH}_{4} \rightarrow \mathrm{HI}+\mathrm{CH}_{3}$ & $3.82 \times 10^{-11} \exp (-16365 / T)$ & (Mečiarová et al., 2011) & $\mathrm{I}+\mathrm{CH} 4 \rightarrow \mathrm{HI}+\mathrm{MR}$ \\
\hline 9 & $\mathrm{I}+$ benzene $\rightarrow \mathrm{HI}+$ phenyl & $1.10 \times 10^{-8} \exp (-21540 / T)$ & (Rodgers et al., 1967) & $\mathrm{I}+\mathrm{CSL} \rightarrow \mathrm{HI}+\mathrm{CSLR}$ \\
\hline 10 & $\mathrm{I}+$ phenyl $\rightarrow \mathrm{HI}+$ cyclopentadienyl & $4.78 \times 10^{-13} \exp (-5373 / T)$ & (Furuyama et al., 1971) & $\mathrm{I}+\mathrm{DIEN} \rightarrow \mathrm{HI}+\mathrm{DIENR}$ \\
\hline 11 & $\mathrm{I}+\mathrm{CH}_{3} \mathrm{CH}_{3} \rightarrow \mathrm{HI}+\mathrm{CH}_{3} \mathrm{CH}_{2}$ & $2.21 \times 10^{-10} \exp (-13237 / T)$ & (Knox and Musgrave, 1967) & $\mathrm{I}+\mathrm{ETH} \rightarrow \mathrm{HI}+\mathrm{ETHR}$ \\
\hline 12 & $\mathrm{I}+\mathrm{CH}_{3} \mathrm{C} \equiv \mathrm{CH} \rightarrow \mathrm{HI}+\mathrm{CH}_{3} \mathrm{C} \equiv \mathrm{C}$ & $3.24 \times 10^{-10} \exp (-12154 / T)$ & (Walsh, 1971) & $\mathrm{I}+\mathrm{HC} 3 \rightarrow \mathrm{HI}+\mathrm{HC} 3 \mathrm{R}$ \\
\hline 13 & $\mathrm{I}+\mathrm{CH}_{3} \mathrm{OCH}_{3} \rightarrow \mathrm{HI}+\mathrm{CH}_{3} \mathrm{OCH}_{2}$ & $5.25 \times 10^{-10} \exp (-11706 / T)$ & (Cruickshank and Benson, 1969b) & $\mathrm{I}+\mathrm{HC} 3 \rightarrow \mathrm{HI}+\mathrm{HC} 3 \mathrm{R}$ \\
\hline 14 & $\mathrm{I}+\mathrm{CH}_{3} \mathrm{OH} \rightarrow \mathrm{HI}+\mathrm{CH}_{2} \mathrm{OH}$ & $5.25 \times 10^{-10} \exp (-13116 / T)$ & (Cruickshank and Benson, 1969a) & $\mathrm{I}+\mathrm{HC} 3 \rightarrow \mathrm{HI}+\mathrm{HC} 3 \mathrm{R}$ \\
\hline 15 & $\mathrm{I}+$ cyclobutane $\rightarrow \mathrm{HI}+$ cyclobutyl & $7.59 \times 10^{-10} \exp (-13477 / T)$ & (McMillen et al., 1972) & $\mathrm{I}+\mathrm{HC} 3 \rightarrow \mathrm{HI}+\mathrm{HC} 3 \mathrm{R}$ \\
\hline 16 & $\mathrm{I}+\mathrm{i}-\mathrm{C}_{4} \mathrm{H}_{10} \rightarrow \mathrm{HI}+\mathrm{t}-\mathrm{C}_{4} \mathrm{H}_{9}$ & $3.32 \times 10^{-10} \exp (-11305 / T)$ & (Knox and Musgrave, 1967) & $\mathrm{I}+\mathrm{HC} 3 \rightarrow \mathrm{HI}+\mathrm{HC} 3 \mathrm{R}$ \\
\hline 17 & $\mathrm{I}+\mathrm{CH}_{3} \mathrm{CH}_{2} \mathrm{CH}_{3} \rightarrow \mathrm{HI}+\mathrm{i}-\mathrm{C}_{3} \mathrm{H}_{7}$ & $1.83 \times 10^{-10} \exp (-12006 / T)$ & (Knox and Musgrave, 1967) & $\mathrm{I}+\mathrm{HC} 3 \rightarrow \mathrm{HI}+\mathrm{HC} 3 \mathrm{R}$ \\
\hline 18 & $\mathrm{I}+\mathrm{CH}_{3} \mathrm{CH}_{2} \mathrm{CH}_{3} \rightarrow \mathrm{HI}+\mathrm{n}-\mathrm{C}_{3} \mathrm{H}_{7}$ & $1.26 \times 10^{-9} \exp (-14921 / T)$ & (Knox and Musgrave, 1967) & $\mathrm{I}+\mathrm{HC} 3 \rightarrow \mathrm{HI}+\mathrm{HC} 3 \mathrm{R}$ \\
\hline 19 & $\mathrm{I}+$ cyclopropane $\rightarrow \mathrm{CH}_{2} \mathrm{CH}_{2} \mathrm{CH}_{2} \mathrm{I}$ & $1.22 \times 10^{-11} \exp (-8705 / T)$ & (Ogg and Priest, 1939) & $\mathrm{I}+\mathrm{HC} 3 \rightarrow \mathrm{I}{ }_{-} \mathrm{HC} 3 \mathrm{R}$ \\
\hline 20 & $\mathrm{I}+$ cyclopentane $\rightarrow \mathrm{HI}+$ cyclopentyl & $1.48 \times 10^{-9} \exp (-12515 / T)$ & (Furuyama et al., 1970b) & $\mathrm{I}+\mathrm{HC} 5 \rightarrow \mathrm{HI}+\mathrm{HC} 5 \mathrm{R}$ \\
\hline 21 & $\mathrm{I}+\mathrm{i}-\mathrm{C}_{3} \mathrm{H}_{7} \mathrm{OH} \rightarrow \mathrm{HI}+\mathrm{CH}_{3} \mathrm{CHOCH}_{3}$ & $1.94 \times 10^{-13} \exp (-10305 / T)$ & (Walsh and Benson, 1966b) & $\mathrm{I}+\mathrm{HC} 5 \rightarrow \mathrm{HI}+\mathrm{HC} 3 \mathrm{R}$ \\
\hline
\end{tabular}




\begin{tabular}{|c|c|c|c|c|}
\hline \# & Reaction & $\begin{array}{c}\text { Rate coefficient } \\
\left(\mathrm{s}^{-1}\right) \text { or }\left(\mathrm{cm}^{3} \text { molecule } \mathrm{s}^{-1}\right) \\
\text { or }\left(\mathrm{cm}^{6} \mathrm{molecule}^{-2} \mathrm{~s}^{-1}\right)\end{array}$ & Reference & IRACM reaction \\
\hline 22 & $\mathrm{I}+$ tetrahydrofurane $\rightarrow \mathrm{HI}+$ tetrahydrofuranyl & $7.77 \times 10^{-10} \exp (-10814 / T)$ & (Cruickshank and Benson, 1969c) & $\mathrm{I}+\mathrm{HC} 8 \rightarrow \mathrm{HI}+\mathrm{HC} 8 \mathrm{R}$ \\
\hline 23 & $\mathrm{I}+\mathrm{HC}(\mathrm{O}) \mathrm{H} \rightarrow \mathrm{HI}+\mathrm{HC}(\mathrm{O})$ & $1.38 \times 10^{-10} \exp (-8774 / T)$ & (Walsh and Benson, 1966a) & $\mathrm{I}+\mathrm{HCHO} \rightarrow \mathrm{HI}+\mathrm{HCHOR}$ \\
\hline 24 & $\mathrm{I}+\mathrm{CH}_{3} \mathrm{CH}_{2} \mathrm{C}(\mathrm{O}) \mathrm{CH}_{3} \rightarrow \mathrm{HI}+\mathrm{CH}_{3} \mathrm{CHC}(\mathrm{O}) \mathrm{CH}_{3}$ & $9.47 \times 10^{-11} \exp (-11066 / T)$ & (Solly et al., 1970a) & $\mathrm{I}+\mathrm{KET} \rightarrow \mathrm{HI}+\mathrm{KETR}$ \\
\hline 25 & $\mathrm{I}+\mathrm{CH}_{3} \mathrm{C}(\mathrm{O}) \mathrm{CH}_{3} \rightarrow \mathrm{HI}+\mathrm{CH}_{2} \mathrm{C}(\mathrm{O}) \mathrm{CH}_{3}$ & $2.62 \times 10^{-10} \exp (-13959 / T)$ & (Solly et al., 1970b) & $\mathrm{I}+\mathrm{KET} \rightarrow \mathrm{HI}+\mathrm{KETR}$ \\
\hline 26 & $\mathrm{I}+\mathrm{CH}_{3} \mathrm{O}_{2} \rightarrow \mathrm{CH}_{3} \mathrm{OOI}$ & $2.00 \times 10^{-11}$ & (Dillon et al., 2006) & $\mathrm{I}+\mathrm{MO} 2 \rightarrow \mathrm{I} \_\mathrm{OP} 1$ \\
\hline 27 & $\mathrm{I}+$ cyclopentene $\rightarrow \mathrm{HI}+$ cyclopentyl & $2.96 \times 10^{-11} \exp (-6161 / T)$ & (Furuyama et al., 1970a) & $\mathrm{I}+\mathrm{OLI} \rightarrow \mathrm{HI}+\mathrm{OLIR}$ \\
\hline 28 & $\mathrm{I}+\mathrm{CH}_{2}=\mathrm{CHCH}_{2} \mathrm{OH} \rightarrow \mathrm{HI}+\mathrm{CH}_{2}=\mathrm{CHCHOH}$ & $5.25 \times 10^{-12} \exp (-5691 / T)$ & (Alfassi and Golden, 1973a) & $\mathrm{I}+\mathrm{OLT} \rightarrow \mathrm{HI}+\mathrm{OLTR}$ \\
\hline 29 & $\mathrm{I}+\mathrm{CH}_{2}=\mathrm{CHCH}_{3} \rightarrow \mathrm{HI}+\mathrm{CH}_{2}=\mathrm{CHCH}_{2}$ & $2.96 \times 10^{-11} \exp (-9085 / T)$ & (Golden et al., 1966) & $\mathrm{I}+\mathrm{OLT} \rightarrow \mathrm{HI}+\mathrm{OLTR}$ \\
\hline 30 & $\mathrm{I}+$ toluene $\rightarrow \mathrm{HI}+$ benzyl & $4.47 \times 10^{-13} \exp (-72539 / T)$ & (Walsh et al., 1966) & $\mathrm{I}+\mathrm{TOL} \rightarrow \mathrm{HI}+\mathrm{TOLR}$ \\
\hline 31 & $\mathrm{IO}+\mathrm{C}_{2} \mathrm{H}_{4} \rightarrow$ products & $2.00 \times 10^{-16}$ & (Maguin et al., 1991) & $\mathrm{IO}+\mathrm{ETE} \rightarrow \mathrm{PROD}$ \\
\hline 32 & $\mathrm{IO}+\mathrm{C}_{2} \mathrm{H}_{5} \mathrm{O}_{2} \rightarrow$ products & $1.40 \times 10^{-10}$ & (Enami et al., 2006) & $\mathrm{IO}+\mathrm{ETHP} \rightarrow \mathrm{PROD}$ \\
\hline 33 & $\mathrm{IO}+\mathrm{CH}_{3} \mathrm{O}_{2} \rightarrow$ products & $1.30 \times 10^{-10} \exp (-168 / T)$ & (Enami et al., 2006) & $\mathrm{IO}+\mathrm{MO} 2 \rightarrow \mathrm{PROD}$ \\
\hline 34 & $\mathrm{IO}+\mathrm{CH}_{2}=\mathrm{CHCH}_{3} \rightarrow$ products & $2.00 \times 10^{-16}$ & (Maguin et al., 1991) & $\mathrm{IO}+\mathrm{OLT} \rightarrow \mathrm{PROD}$ \\
\hline 35 & $\mathrm{IO}+\mathrm{HCOOH} \rightarrow \mathrm{HOI}+\mathrm{HCOO}$ & $2.91 \times 10^{-15}$ & (Buben et al., 1995) & $\mathrm{IO}+\mathrm{ORA} 1 \rightarrow \mathrm{HOI}+\mathrm{ORA} 1 \mathrm{R}$ \\
\hline 36 & $\mathrm{I}_{2}+$ cyclopentane $\rightarrow 2 \mathrm{HI}+$ cyclopentyl & $1.48 \times 10^{-9} \exp (-12515 / T)$ & (Furuyama et al., 1970b) & $\mathrm{I} 2+\mathrm{HC} 5 \rightarrow 2 \mathrm{HI}+\mathrm{HC} 5 \mathrm{R}$ \\
\hline 37 & $\mathrm{CH}_{3} \mathrm{C}(\mathrm{O}) \mathrm{I} \rightarrow \mathrm{I}+\mathrm{CH}_{3} \mathrm{C}(\mathrm{O})$ & $1.00 \times 10^{13} \exp (-25511 / T)$ & (Butler and Polanyi, 1943) & I_ALD $\rightarrow$ I + ALDR \\
\hline 38 & $\mathrm{CH}_{3} \mathrm{I}+\mathrm{H} \rightarrow \mathrm{HI}+\mathrm{CH}_{3}$ & $6.30 \times 10^{-11} \exp (-602 / T)$ & (Yuan et al., 1997) & $\mathrm{I} \_\mathrm{CH} 4+\mathrm{H} \rightarrow \mathrm{HI}+\mathrm{MR}$ \\
\hline 39 & $\mathrm{CH}_{3} \mathrm{I}+\mathrm{OH} \rightarrow \mathrm{CH}_{2} \mathrm{I}+\mathrm{H}_{2} \mathrm{O}$ & $4.10 \times 10^{-12} \exp (-1239 / T)$ & (Zhang et al., 2011b) & $\mathrm{I} \_\mathrm{CH} 4+\mathrm{H} \rightarrow \mathrm{I} \_\mathrm{MR}+\mathrm{H} 2 \mathrm{O}$ \\
\hline 40 & $\mathrm{CH}_{3} \mathrm{I}+\mathrm{NO}_{3} \rightarrow \mathrm{CH}_{2} \mathrm{I}+\mathrm{HNO}_{3}$ & $9.10 \times 10^{-11} \exp (-1564 / T)$ & (Nakano et al., 2008) & $\mathrm{I} \_\mathrm{CH} 4+\mathrm{NO} 3 \rightarrow \mathrm{I} \_\mathrm{MR}+\mathrm{HNO} 3$ \\
\hline
\end{tabular}




\begin{tabular}{|c|c|c|c|c|}
\hline \# & Reaction & $\begin{array}{c}\text { Rate coefficient } \\
\left(\mathrm{s}^{-1}\right) \text { or }\left(\mathrm{cm}^{3} \text { molecule }^{-1} \mathrm{~s}^{-1}\right) \\
\text { or }\left(\mathrm{cm}^{6} \text { molecule }^{-2} \mathrm{~s}^{-1}\right)\end{array}$ & Reference & IRACM reaction \\
\hline \multirow{5}{*}{41} & $\mathrm{CH}_{3} \mathrm{I}+\mathrm{O}\left({ }^{3} \mathrm{P}\right) \rightarrow \mathrm{IO}+\mathrm{CH}_{3}$ & $1.00 \times 10^{-11} \exp (160 / T) \times 0.59$ & \multirow{5}{*}{ (Gilles et al., 1996) } & I_CH4 + O3P $\rightarrow \mathrm{IO}+\mathrm{MR}$ \\
\hline & $\rightarrow \mathrm{CH}_{2} \mathrm{I}+\mathrm{OH}$ & $1.00 \times 10^{-11} \exp (160 / T) \times 0.21$ & & $\rightarrow \mathrm{I} \_\mathrm{MR}+\mathrm{HO}$ \\
\hline & $\rightarrow \mathrm{H}+\mathrm{I}+\mathrm{HC}(\mathrm{O}) \mathrm{H}$ & $1.00 \times 10^{-11} \exp (160 / T) \times 0.09$ & & \multirow{3}{*}{$\begin{array}{l}\rightarrow \mathrm{H}+\mathrm{I}+\mathrm{HCHO} \\
\rightarrow \mathrm{I}+\mathrm{HC} 3 \mathrm{R} \\
\rightarrow \mathrm{HI}+\mathrm{HCHO}\end{array}$} \\
\hline & $\rightarrow \mathrm{I}+\mathrm{CH}_{3} \mathrm{O}$ & $1.00 \times 10^{-11} \exp (160 / T) \times 0.04$ & & \\
\hline & $\rightarrow \mathrm{HI}+\mathrm{HC}(\mathrm{O}) \mathrm{H}$ & $1.00 \times 10^{-11} \exp (160 / T) \times 0.07$ & & \\
\hline 42 & $\mathrm{CH}_{3} \mathrm{I} \rightarrow \mathrm{I}+\mathrm{CH}_{3}$ & $5.62 \times 10^{13} \exp (-27797 / T)$ & (Skorobogatov et al., 1994) & I_CH4 $\rightarrow$ I + MR \\
\hline 43 & iodobenzene $+\mathrm{H} \rightarrow \mathrm{HI}+$ phenyl & $1.45 \times 10^{-18} \mathrm{~T}^{2.5} \exp (73 / T)$ & (Gao et al., 2008) & $\mathrm{I} \_\mathrm{CSL}+\mathrm{H} \rightarrow \mathrm{HI}+\mathrm{CSLR}$ \\
\hline 44 & iodobenzene $+\mathrm{OH} \rightarrow$ products & $1.32 \times 10^{-12}$ & (Wallington et al., 1987) & I_CSL + HO $\rightarrow$ PROD \\
\hline 45 & iodobenzene $\rightarrow$ products & $1.98 \times 10^{11} \exp (-23104 / T)$ & (Kumaran et al., 1997) & I_CSL $\rightarrow$ PROD \\
\hline 46 & $\mathrm{CH}_{2}=\mathrm{CHI} \rightarrow \mathrm{I}+\mathrm{CH}_{2}=\mathrm{CH}$ & $4.00 \times 10^{15} \exp (-33574 / T)$ & (Shilov and Sabirova, 1964) & I_ETE $\rightarrow$ I + ETER \\
\hline 47 & $\mathrm{CH}_{3} \mathrm{CH}_{2} \mathrm{I}+\mathrm{H} \rightarrow \mathrm{CH}_{3} \mathrm{CH}_{2}+\mathrm{HI}$ & $1.10 \times 10^{-10} \exp (-708 / T)$ & (Yuan et al., 1997) & I_ETH + H $\rightarrow$ ETHR + HI \\
\hline 48 & $\mathrm{CH}_{3} \mathrm{CH}_{2} \mathrm{I}+\mathrm{OH} \rightarrow \mathrm{CH}_{2} \mathrm{CH}_{2} \mathrm{I}+\mathrm{H}_{2} \mathrm{O}$ & $5.55 \times 10^{-12} \exp (-830 / T)$ & (Zhang et al., 2012) & I_ETH + HO $\rightarrow$ I_ETHR + H2O \\
\hline 49 & $\mathrm{CH}_{3} \mathrm{CH}_{2} \mathrm{I}+\mathrm{O}\left({ }^{3} \mathrm{P}\right) \rightarrow$ products & $1.58 \times 10^{-11} \exp (239 / T)$ & (Teruel et al., 2004) & I_ETH + O3P $\rightarrow$ PROD \\
\hline 50 & $\mathrm{CH}_{3} \mathrm{CH}_{2} \mathrm{I} \rightarrow \mathrm{CH}_{3} \mathrm{CH}_{2}+\mathrm{I}$ & $6.34 \times 10^{9} \exp (-15884 / T)$ & (Kumaran et al., 1996) & I_ETH $\rightarrow$ ETHR + I \\
\hline 51 & $\mathrm{n}-\mathrm{C}_{3} \mathrm{H}_{7} \mathrm{I}+\mathrm{H} \rightarrow \mathrm{n}-\mathrm{C}_{3} \mathrm{H}_{7}+\mathrm{HI}$ & $1.00 \times 10^{-13}$ & (Rebbert et al., 1973) & $\mathrm{I} \_\mathrm{HC} 3+\mathrm{H} \rightarrow \mathrm{HC} 3 \mathrm{R}+\mathrm{HI}$ \\
\hline 52 & $\mathrm{i}-\mathrm{C}_{3} \mathrm{H}_{7} \mathrm{I}+\mathrm{H} \rightarrow \mathrm{i}-\mathrm{C}_{3} \mathrm{H}_{7}+\mathrm{HI}$ & $1.40 \times 10^{-11}$ & (Yuan et al., 1997) & $\mathrm{I} \_\mathrm{HC} 3+\mathrm{H} \rightarrow \mathrm{HC} 3 \mathrm{R}+\mathrm{HI}$ \\
\hline 53 & $\mathrm{t}-\mathrm{C}_{3} \mathrm{H}_{7} \mathrm{I}+\mathrm{H} \rightarrow \mathrm{t}-\mathrm{C}_{3} \mathrm{H}_{7}+\mathrm{HI}$ & $2.00 \times 10^{-11}$ & (Yuan et al., 1997) & $\mathrm{I} \_\mathrm{HC} 3+\mathrm{H} \rightarrow \mathrm{HC} 3 \mathrm{R}+\mathrm{HI}$ \\
\hline \multirow{3}{*}{54} & $\mathrm{CH}_{2} \mathrm{ICH}_{2} \mathrm{CH}_{3}+\mathrm{OH} \rightarrow \mathrm{CH}_{2} \mathrm{CH}_{2} \mathrm{CH}_{2} \mathrm{I}+\mathrm{H}_{2} \mathrm{O}$ & $1.65 \times 10^{-11} \exp (-781 / T) \times 0.09^{\mathrm{a}}$ & \multirow{3}{*}{ (Zhang et al., 2012) } & $\mathrm{I} \_\mathrm{HC} 3+\mathrm{HO} \rightarrow \mathrm{I} \_\mathrm{HC} 3 \mathrm{R}+\mathrm{H} 2 \mathrm{O}$ \\
\hline & $\rightarrow \mathrm{CH}_{3} \mathrm{CH}_{2} \mathrm{CHI}+\mathrm{H}_{2} \mathrm{O}$ & $1.65 \times 10^{-11} \exp (-781 / T) \times 0.37^{\mathrm{a}}$ & & $\rightarrow \mathrm{I} \_\mathrm{HC} 3 \mathrm{R}+\mathrm{H} 2 \mathrm{O}$ \\
\hline & $\rightarrow \mathrm{CH}_{3} \mathrm{CHCH}_{2} \mathrm{I}+\mathrm{H}_{2} \mathrm{O}$ & $1.65 \times 10^{-11} \exp (-781 / T) \times 0.54^{\mathrm{a}}$ & & $\rightarrow \mathrm{I} \_\mathrm{HC} 3 \mathrm{R}+\mathrm{H} 2 \mathrm{O}$ \\
\hline \multirow{2}{*}{55} & $\mathrm{CH}_{3} \mathrm{CHICH}_{3}+\mathrm{OH} \rightarrow \mathrm{CH}_{2} \mathrm{CHICH}_{3}+\mathrm{H}_{2} \mathrm{O}$ & $7.58 \times 10^{-12} \exp (-531 / T) \times 0.22^{\mathrm{a}}$ & \multirow{2}{*}{ (Zhang et al., 2012) } & $\mathrm{I} \_\mathrm{HC} 3+\mathrm{HO} \rightarrow \mathrm{I} \_\mathrm{HC} 3 \mathrm{R}+\mathrm{H} 2 \mathrm{O}$ \\
\hline & $\rightarrow \mathrm{CH}_{3} \mathrm{CICH}_{3}+\mathrm{H}_{2} \mathrm{O}$ & $7.58 \times 10^{-12} \exp (-531 / T) \times 0.78^{(}$ & & $\rightarrow \mathrm{I} \_\mathrm{HC} 3 \mathrm{R}+\mathrm{H} 2 \mathrm{O}$ \\
\hline
\end{tabular}




\begin{tabular}{|c|c|c|c|c|}
\hline$\overline{\#}$ & Reaction & $\begin{array}{c}\text { Rate coefficient } \\
\left(\mathrm{s}^{-1}\right) \text { or }\left(\mathrm{cm}^{3} \text { molecule }{ }^{-1} \mathrm{~s}^{-1}\right) \\
\text { or }\left(\mathrm{cm}^{6} \text { molecule } \mathrm{s}^{-1}\right)\end{array}$ & Reference & IRACM reaction \\
\hline \multirow{2}{*}{56} & $\mathrm{CH}_{2} \mathrm{ICH}_{2} \mathrm{CH}_{3}+\mathrm{O}\left({ }^{3} \mathrm{P}\right) \rightarrow \mathrm{HOI}+\mathrm{CH}_{2}=\mathrm{CHCH}_{3}$ & $1.10 \times 10^{-11} \exp (367 / T) \times 0.79$ & \multirow{2}{*}{ (Teruel et al., 2004) } & I_HC3 + O3P $\rightarrow$ HIO + OLT \\
\hline & $\rightarrow \mathrm{IO}+\mathrm{CH}_{2} \mathrm{CH}_{2} \mathrm{CH}_{3}$ & $1.10 \times 10^{-11} \exp (367 / T) \times 0.21$ & & $\rightarrow \mathrm{IO}+\mathrm{HC} 3 \mathrm{R}$ \\
\hline \multirow{2}{*}{57} & $\mathrm{CH}_{3} \mathrm{CHICH}_{3}+\mathrm{O}\left({ }^{3} \mathrm{P}\right) \rightarrow \mathrm{HOI}+\mathrm{CH}_{2}=\mathrm{CHCH}_{3}$ & $1.84 \times 10^{-11} \exp (296 / T) \times 0.79$ & \multirow{2}{*}{ (Teruel et al., 2004) } & $\mathrm{I} \_\mathrm{HC} 3+\mathrm{O} 3 \mathrm{P} \rightarrow \mathrm{HIO}+\mathrm{OLT}$ \\
\hline & $\rightarrow \mathrm{IO}+\mathrm{CH}_{3} \mathrm{CHCH}_{3}$ & $1.84 \times 10^{-11} \exp (296 / T) \times 0.21$ & & $\rightarrow \mathrm{IO}+\mathrm{HC} 3 \mathrm{R}$ \\
\hline 58 & $\mathrm{~s}-\mathrm{C}_{4} \mathrm{H}_{9} \mathrm{I} \rightarrow \mathrm{HI}+\mathrm{CH}_{3} \mathrm{CH}=\mathrm{CHCH}_{3}$ & $1.58 \times 10^{15} \exp (-24067 / T)$ & (Holmes and Maccoll, 1963) & I_HC3 $\rightarrow$ HI + OLT \\
\hline 59 & $\mathrm{i}-\mathrm{C}_{3} \mathrm{H}_{7} \mathrm{I} \rightarrow \mathrm{HI}+\mathrm{CH}_{2}=\mathrm{CHCH}_{3}$ & $4.68 \times 10^{13} \exp (-22623 / T)$ & (Tsang, 1964) & I_HC3 $\rightarrow$ HI + OLT \\
\hline 60 & $\mathrm{t}-\mathrm{C}_{4} \mathrm{H}_{9} \mathrm{I} \rightarrow \mathrm{HI}+\mathrm{i}-\mathrm{C}_{4} \mathrm{H}_{8}$ & $7.94 \times 10^{13} \exp (-19735 / T)$ & (Hippler et al., 1991) & I_HC3 $\rightarrow$ HI + OLT \\
\hline 61 & $\mathrm{CH}_{2} \mathrm{CH}_{2} \mathrm{CH}_{2} \mathrm{I} \rightarrow \mathrm{I}+$ cyclopropane & $3.31 \times 10^{-11} \exp (-8104 / T)$ & (Benson, 1961) & I_HC3R $\rightarrow$ I + I_HC3 \\
\hline 62 & iodocyclopentane $\rightarrow \mathrm{HI}+$ cyclopentene & $2.00 \times 10^{13}$ & (Furuyama et al., 1970b) & I_HC5 $\rightarrow$ HI + OLI \\
\hline 63 & $\mathrm{CH}_{2} \mathrm{I}+\mathrm{NO}_{2} \rightarrow$ products & $8.51 \times 10^{-8} T^{-1.45}$ & (Eskola et al., 2006) & I_MR $+\mathrm{NO} 2 \rightarrow$ PROD \\
\hline 64 & $\mathrm{CH}_{2} \mathrm{I}+\mathrm{O}_{2} \rightarrow \mathrm{CH}_{2} \mathrm{IO}_{2}$ & $1.82 \times 10^{-12}$ & (Sheps, 2013) & I_MR + O2 $\rightarrow$ I_MO2 \\
\hline 65 & $\mathrm{CH}_{2} \mathrm{I}+\mathrm{O}_{2} \rightarrow \mathrm{HC}(\mathrm{O}) \mathrm{H}+\mathrm{IO}$ & $4.00 \times 10^{-13}$ & (Enami et al., 2004) & $\mathrm{I} \_\mathrm{MR}+\mathrm{O} 2 \rightarrow \mathrm{HCHO}+\mathrm{IO}$ \\
\hline 66 & $\mathrm{CH}_{2}=\mathrm{CHCH}_{2} \mathrm{I}+\mathrm{OH} \rightarrow$ products & $6.00 \times 10^{-11}$ & (Albaladejo et al., 2003) & I_OLT $+\mathrm{HO} \rightarrow$ PROD \\
\hline 67 & $\mathrm{CH}_{2}=\mathrm{CHCH}_{2} \mathrm{I}+\mathrm{NO}_{3} \rightarrow$ products & $3.91 \times 10^{-14}$ & (Martinez et al., 1996) & I_OLT + NO3 $\rightarrow$ PROD \\
\hline 68 & $\mathrm{CH}_{2}=\mathrm{CHCH}_{2} \mathrm{I}+\mathrm{O}_{3} \rightarrow$ products & $8.17 \times 10^{-14} \exp (-2992 / T)$ & (Gai et al., 2009) & I_OLT + O3 $\rightarrow$ PROD \\
\hline 69 & $\mathrm{CH}_{2}=\mathrm{CHCH}_{2} \mathrm{I} \rightarrow \mathrm{I}+\mathrm{CH}_{2}=\mathrm{CHCH}_{2}$ & $1.00 \times 10^{13} \exp (-19614 / T)$ & (Butler and Polanyi, 1943) & I_OLT $\rightarrow$ I + OLTR \\
\hline 70 & iodomethylbenzene $\rightarrow$ I + benzyl & $6.03 \times 10^{14}$ & (Mueller-Markgraf and Troe, & I_TOL $\rightarrow$ I + TOLR \\
\hline 71 & $\mathrm{CH}_{2} \mathrm{I}_{2}+\mathrm{NO}_{3} \rightarrow$ products & $4.00 \times 10^{-13}$ & (Nakano et al., 2006) & I2_CH4 + NO3 $\rightarrow$ PROD \\
\hline 72 & $\mathrm{CH}_{2} \mathrm{I}_{2}+\mathrm{O}\left({ }^{3} \mathrm{P}\right) \rightarrow$ products & $7.36 \times 10^{-11}$ & (Teruel et al., 2004) & I2_CH4 + O3P $\rightarrow$ PROD \\
\hline 73 & $\mathrm{CH}_{2} \mathrm{I}_{2} \rightarrow \mathrm{I}+\mathrm{CH}_{2} \mathrm{I}$ & $2.51 \times 10^{15} \exp (-21299 / T)$ & (Saito et al., 1980) & I2_CH4 $\rightarrow$ I + I_MR \\
\hline 74 & $\mathrm{CHI}=\mathrm{CHI} \rightarrow \mathrm{I}_{2}+\mathrm{C}_{2} \mathrm{H}_{2}$ & $3.11 \times 10^{12} \exp (-23104 / T)$ & (Furuyama et al., 1969c) & I2_ETE $\rightarrow$ I $2+\mathrm{HC} 3$ \\
\hline 75 & $\mathrm{CHI}_{3} \rightarrow \mathrm{I}+\mathrm{CHI}_{2}$ & $1.00 \times 10^{13} \exp (-18652 / T)$ & (Butler et al., 1945) & I3_CH4 $\rightarrow$ I + I2_MR \\
\hline
\end{tabular}




\begin{tabular}{|c|c|c|c|c|}
\hline \# & Reaction & $\begin{array}{c}\text { Rate coefficient } \\
\left(\mathrm{s}^{-1}\right) \text { or }\left(\mathrm{cm}^{3} \text { molecule } \mathrm{e}^{-1} \mathrm{~s}^{-1}\right) \\
\text { or }\left(\mathrm{cm}^{6} \text { molecule }^{-2} \mathrm{~s}^{-1}\right)\end{array}$ & Reference & IRACM reaction \\
\hline 76 & $\mathrm{CH}_{3} \mathrm{CH}_{2} \mathrm{I}+\mathrm{NO}_{3} \rightarrow$ products & $9.48 \times 10^{-27} \exp (-654 / T)$ & (Bai et al., 2015) & I_ETH + NO3 $\rightarrow$ PROD \\
\hline 77 & $\mathrm{CH}_{2} \mathrm{I}_{2}+\mathrm{OH} \rightarrow$ products & $4.20 \times 10^{-11} \exp (-670 / T)$ & (Zhang et al., 2011a) & I2_CH4 + HO $\rightarrow$ PROD \\
\hline 78 & $\mathrm{CH}_{3} \mathrm{CHI}+\mathrm{O}_{2} \rightarrow \mathrm{CH}_{3} \mathrm{CHO}_{2}+\mathrm{I}$ & $8.00 \times 10^{-12}$ & (Sheps et al., 2014) & $\mathrm{I} \_\mathrm{ETHR}+\mathrm{O} 2 \rightarrow \mathrm{ETHP}+\mathrm{I}$ \\
\hline 79 & $\mathrm{HI}+\mathrm{CH}_{2} \mathrm{I} \rightarrow \mathrm{I}+\mathrm{CH}_{3} \mathrm{I}$ & $1.69 \times 10^{-12} \exp (192 / T)$ & (Seetula and Gutman, 1991) & $\mathrm{HI}+\mathrm{I} \_\mathrm{MR} \rightarrow \mathrm{I}+\mathrm{I} \_\mathrm{CH} 4$ \\
\hline 80 & $\mathrm{I}+\mathrm{CH}_{3} \mathrm{C}(\mathrm{O}) \mathrm{I} \rightarrow \mathrm{I}_{2}+\mathrm{CH}_{3} \mathrm{C}(\mathrm{O})$ & $1.32 \times 10^{-10} \exp (-7353 / T)$ & (O'Neal and Benson, 1962b) & $\mathrm{I}+\mathrm{I} \_\mathrm{ALD} \rightarrow \mathrm{I} 2+$ ALDR \\
\hline 81 & $\mathrm{I}+\mathrm{CH}_{3} \mathrm{I} \rightarrow \mathrm{I}_{2}+\mathrm{CH}_{3}$ & $4.17 \times 10^{-10} \exp (-1030 / T)$ & (Flowers and Benson, 1963) & $\mathrm{I}+\mathrm{I} \_\mathrm{CH} 4 \rightarrow \mathrm{I} 2+\mathrm{MR}$ \\
\hline 82 & $\mathrm{I}+$ iodobenzene $\rightarrow \mathrm{I}_{2}+$ phenyl & $3.80 \times 10^{-10} \exp (-14320 / T)$ & (Rodgers et al., 1967) & $\mathrm{I}+\mathrm{I} \_\mathrm{CSL} \rightarrow \mathrm{I} 2+\mathrm{CSLR}$ \\
\hline 83 & $\mathrm{I}+$ iodocyclopentadiene $\rightarrow \mathrm{I}_{2}+$ cyclopentadienyl & $2.09 \times 10^{-12} \exp (-204 / T)$ & (Furuyama et al., 1971) & I + I_DIEN $\rightarrow$ I 2 + DIENR \\
\hline 84 & $\mathrm{I}+\mathrm{CH}_{3} \mathrm{CH}_{2} \mathrm{I} \rightarrow \mathrm{I}_{2}+\mathrm{CH}_{3} \mathrm{CH}_{2}$ & $1.69 \times 10^{-10} \exp (-8604 / T)$ & (Hartley and Benson, 1963) & $\mathrm{I}+\mathrm{I} \_\mathrm{ETH} \rightarrow \mathrm{I} 2+\mathrm{ETHR}$ \\
\hline 85 & $\mathrm{I}+\mathrm{s}-\mathrm{C}_{4} \mathrm{H}_{9} \mathrm{I} \rightarrow \mathrm{HI}+\mathrm{I}+\mathrm{CH}_{3} \mathrm{CH}=\mathrm{CHCH}_{3}$ & $4.90 \times 10^{-12} \exp (-9014 / T)$ & (Nangia and Benson, 1964) & $\mathrm{I}+\mathrm{I} \_\mathrm{HC} 3 \rightarrow \mathrm{HI}+\mathrm{I}+\mathrm{OLI}$ \\
\hline 86 & $\mathrm{I}+$ iodomethylcyclopropane $\rightarrow \mathrm{I}_{2}+$ methylcyclopropyl & $4.17 \times 10^{-10} \exp (-8454 / T)$ & (McMillen et al., 1971) & $\mathrm{I}+\mathrm{I} \_\mathrm{HC} 3 \rightarrow \mathrm{I} 2+\mathrm{HC} 3 \mathrm{R}$ \\
\hline 87 & $\mathrm{I}+\mathrm{n}-\mathrm{C}_{3} \mathrm{H}_{7} \mathrm{I} \rightarrow \mathrm{I}_{2}+\mathrm{n}-\mathrm{C}_{3} \mathrm{H}_{7}$ & $3.25 \times 10^{-10} \exp (-9315 / T)$ & (Benson and O'Neal, 1961) & $\mathrm{I}+\mathrm{I} \_\mathrm{HC} 3 \rightarrow \mathrm{I} 2+\mathrm{HC} 3 \mathrm{R}$ \\
\hline 88 & $\mathrm{I}+\mathrm{t}-\mathrm{C}_{4} \mathrm{H}_{9} \mathrm{I} \rightarrow \mathrm{I}_{2}+\mathrm{t}-\mathrm{C}_{4} \mathrm{H}_{9} \mathrm{I}$ & $8.32 \times 10^{-11} \exp (-6543 / T)$ & (Teranishi and Benson, 1963) & $\mathrm{I}+\mathrm{I} \_\mathrm{HC} 3 \rightarrow \mathrm{I} 2+\mathrm{HC} 3 \mathrm{R}$ \\
\hline 89 & $\mathrm{I}+$ iodocyclopentane $\rightarrow \mathrm{I}_{2}+$ cyclopentyl & $8.32 \times 10^{-10} \exp (-8564 / T)$ & (Furuyama et al., 1970b) & $\mathrm{I}+\mathrm{I} \_\mathrm{HC} 5 \rightarrow \mathrm{I} 2+\mathrm{HC} 5 \mathrm{R}$ \\
\hline 90 & $\mathrm{I}+\mathrm{CH}_{2} \mathrm{I} \rightarrow \mathrm{CH}_{2} \mathrm{I}_{2}$ & $1.33 \times 10^{-10}$ & (Hunter and Kristjansson, 1982) & $\mathrm{I}+\mathrm{I} \_\mathrm{MR} \rightarrow \mathrm{I} 2 \_\mathrm{CH} 4$ \\
\hline 91 & $\mathrm{I}+\mathrm{CH}_{3} \mathrm{OOI} \rightarrow \mathrm{I}_{2}+\mathrm{CH}_{3} \mathrm{O}_{2}$ & $1.50 \times 10^{-10}$ & (Dillon et al., 2006) & $\mathrm{I}+\mathrm{I} \_\mathrm{OP} 1 \rightarrow \mathrm{I} 2+\mathrm{MO} 2$ \\
\hline 92 & $\mathrm{I}+$ iodomethylbenzene $\rightarrow \mathrm{I}_{2}+$ benzyl & $9.33 \times 10^{-13} \exp (-1760 / T)$ & (Walsh et al., 1966) & $\mathrm{I}+\mathrm{I} \_\mathrm{TOL} \rightarrow \mathrm{I} 2+\mathrm{TOLR}$ \\
\hline 93 & $\mathrm{I}+\mathrm{CH}_{2} \mathrm{I}_{2} \rightarrow \mathrm{I}_{2}+\mathrm{CH}_{2} \mathrm{I}$ & $4.68 \times 10^{-10} \exp (-7604 / T)$ & (Furuyama et al., 1969a) & $\mathrm{I}+\mathrm{I} 2 \_\mathrm{CH} 4 \rightarrow \mathrm{I} 2+\mathrm{I} \_\mathrm{MR}$ \\
\hline 94 & $\mathrm{I}+\mathrm{CHI}=\mathrm{CHI} \rightarrow$ products & $1.18 \times 10^{-12} \exp (-5542 / T)$ & (Golden and Benson, 1969) & $\mathrm{I}+\mathrm{I} 2 \_\mathrm{ETE} \rightarrow$ PROD \\
\hline 95 & $\mathrm{I}+\mathrm{ICH}_{2} \mathrm{CH}_{2} \mathrm{CH}_{2} \mathrm{I} \rightarrow \mathrm{I}_{2}+\mathrm{CH}_{2} \mathrm{CH}_{2} \mathrm{CH}_{2} \mathrm{I}$ & $1.66 \times 10^{-10} \exp (-9814 / T)$ & (Benson, 1961) & $\mathrm{I}+\mathrm{I} 2 \_\mathrm{HC} 3 \rightarrow \mathrm{I} 2+\mathrm{I} \_\mathrm{HC} 3 \mathrm{R}$ \\
\hline 96 & $\mathrm{I}+\mathrm{CHI}_{3} \rightarrow \mathrm{I}_{2}+\mathrm{CHI}_{2}$ & $9.33 \times 10^{-10} \exp (-4853 / T)$ & (Furuyama et al., 1969b) & $\mathrm{I}+\mathrm{I} 3 \_\mathrm{CH} 4 \rightarrow \mathrm{I} 2+\mathrm{I} 2 \_\mathrm{MR}$ \\
\hline 97 & $\mathrm{I}_{2}+\mathrm{CH}_{2} \mathrm{CH}_{2} \mathrm{CH}_{2} \mathrm{I} \rightarrow \mathrm{I}+\mathrm{ICH}_{2} \mathrm{CH}_{2} \mathrm{CH}_{2} \mathrm{I}$ & $5.25 \times 10^{-12} \exp (-251 / T)$ & (Benson, 1961) & $\mathrm{I} 2+\mathrm{I} \_\mathrm{HC} 3 \mathrm{R} \rightarrow \mathrm{I}+\mathrm{I} 2 \_\mathrm{HC} 3$ \\
\hline
\end{tabular}




\begin{tabular}{|c|c|c|c|c|}
\hline \# & Reaction & $\begin{array}{c}\text { Rate coefficient } \\
\left(\mathrm{s}^{-1}\right) \text { or }\left(\mathrm{cm}^{3} \text { molecule } \mathrm{e}^{-1} \mathrm{~s}^{-1}\right) \\
\text { or }\left(\mathrm{cm}^{6} \text { molecule } \mathrm{s}^{-1}\right)\end{array}$ & Reference & IRACM reaction \\
\hline 98 & $\mathrm{I}_{2}+\mathrm{CH}_{2} \mathrm{I} \rightarrow \mathrm{I}+\mathrm{CH}_{2} \mathrm{I}_{2}$ & $3.32 \times 10^{-10}$ & (Hunter and Kristjansson, 1982) & $\mathrm{I} 2+\mathrm{I} \_\mathrm{MR} \rightarrow \mathrm{I}+\mathrm{I} 2 \_\mathrm{CH} 4$ \\
\hline 99 & $\mathrm{IO}+\mathrm{CH}_{3} \mathrm{I} \rightarrow \mathrm{HOI}+\mathrm{CH}_{2} \mathrm{I}$ & $6.69 \times 10^{-20} T^{2.09} \exp (-26835 / T)$ & (Cours et al., 2013) & $\mathrm{IO}+\mathrm{I} \_\mathrm{CH} 4 \rightarrow \mathrm{HIO}+\mathrm{I} \_\mathrm{MR}$ \\
\hline 100 & $\begin{array}{l}\mathrm{CH}_{2} \mathrm{IO}_{2}+\mathrm{CH}_{2} \mathrm{IO}_{2} \rightarrow 0.5 \mathrm{CH}_{2} \mathrm{IOH}+0.5 \mathrm{HC}(\mathrm{O}) \mathrm{I}+ \\
\mathrm{CH}_{2} \mathrm{IO}+\mathrm{O}_{2}\end{array}$ & $9.00 \times 10^{-11}$ & (Sehested et al., 1994) & $\begin{array}{l}\text { I_MO2 + I_MO2 } \rightarrow 0.5 \text { I_HC3 + } \\
0.5 \text { I_HCHO +I_HC3R }+ \text { O2 }\end{array}$ \\
\hline 101 & $\mathrm{HI}+\mathrm{CH}_{3} \mathrm{C}(\mathrm{O}) \rightarrow \mathrm{I}+\mathrm{CH}_{3} \mathrm{CH}(\mathrm{O})$ & $2.25 \times 10^{-12} \exp (-754 / T)$ & (O'Neal and Benson, 1962a) & $\mathrm{HI}+\mathrm{ALDR} \rightarrow \mathrm{I}+\mathrm{ALD}$ \\
\hline 102 & $\mathrm{HI}+$ phenyl $\rightarrow \mathrm{I}+$ benzene & $2.89 \times 10^{-12} \exp (-200 / T)$ & (Rodgers et al., 1967) & $\mathrm{HI}+\mathrm{CSLR} \rightarrow \mathrm{I}+\mathrm{CSL}$ \\
\hline 103 & $\mathrm{HI}+$ cyclopentadienyl $\rightarrow \mathrm{I}+$ cyclopentadiene & $5.25 \times 10^{-14} \exp (-503 / T)$ & (Furuyama et al., 1971) & $\mathrm{HI}+\mathrm{DIENR} \rightarrow \mathrm{I}+\mathrm{DIEN}$ \\
\hline 104 & $\mathrm{HI}+\mathrm{C}_{2} \mathrm{H}_{3} \rightarrow \mathrm{I}+\mathrm{C}_{2} \mathrm{H}_{4}$ & $7.26 \times 10^{-12} \exp (383 / T)$ & (Seetula, 1991) & $\mathrm{HI}+\mathrm{ETER} \rightarrow \mathrm{I}+\mathrm{ETE}$ \\
\hline 105 & $\mathrm{HI}+\mathrm{CH}_{3} \mathrm{CH}_{2} \rightarrow \mathrm{I}+\mathrm{CH}_{3} \mathrm{CH}_{3}$ & $1.12 \times 10^{-20} T^{2.92} \exp (-429 / T)$ & (Leplat et al., 2015) & $\mathrm{HI}+\mathrm{ETHR} \rightarrow \mathrm{I}+\mathrm{ETH}$ \\
\hline 106 & $\mathrm{HI}+\mathrm{CH}_{2} \mathrm{OH} \rightarrow \mathrm{I}+\mathrm{CH}_{3} \mathrm{OH}$ & $2.71 \times 10^{-12} \exp (-577 / T)$ & (Seetula and Gutman, 1992) & $\mathrm{HI}+\mathrm{HC} 3 \mathrm{R} \rightarrow \mathrm{I}+\mathrm{HC} 3$ \\
\hline 107 & $\mathrm{HI}+\mathrm{i}-\mathrm{C}_{3} \mathrm{H}_{7} \rightarrow \mathrm{I}+\mathrm{CH}_{3} \mathrm{CH}_{2} \mathrm{CH}_{3}$ & $1.94 \times 10^{-11} \exp (-744 / T)$ & (Leplat and Rossi, 2014a) & $\mathrm{HI}+\mathrm{HC} 3 \mathrm{R} \rightarrow \mathrm{I}+\mathrm{HC} 3$ \\
\hline 108 & $\mathrm{HI}+\mathrm{n}-\mathrm{C}_{3} \mathrm{H}_{7} \rightarrow \mathrm{I}+\mathrm{CH}_{3} \mathrm{CH}_{2} \mathrm{CH}_{3}$ & $6.49 \times 10^{-11} \exp (-1333 / T)$ & (Leplat and Rossi, 2014a) & $\mathrm{HI}+\mathrm{HC} 3 \mathrm{R} \rightarrow \mathrm{I}+\mathrm{HC} 3$ \\
\hline 109 & $\mathrm{HI}+\mathrm{s}-\mathrm{C}_{4} \mathrm{H}_{9} \rightarrow \mathrm{I}+\mathrm{n}-\mathrm{C}_{4} \mathrm{H}_{10}$ & $3.20 \times 10^{-12} \exp (708 / T)$ & (Seetula et al., 1990) & $\mathrm{HI}+\mathrm{HC} 3 \mathrm{R} \rightarrow \mathrm{I}+\mathrm{HC} 3$ \\
\hline 110 & $\mathrm{HI}+\mathrm{t}-\mathrm{C}_{4} \mathrm{H}_{9} \rightarrow \mathrm{I}+\mathrm{i}-\mathrm{C}_{4} \mathrm{H}_{10}$ & $2.00 \times 10^{-11} \exp (-1020 / T)$ & (Leplat and Rossi, 2014b) & $\mathrm{HI}+\mathrm{HC} 3 \mathrm{R} \rightarrow \mathrm{I}+\mathrm{HC} 3$ \\
\hline 111 & $\mathrm{HI}+$ cyclopentyl $\rightarrow \mathrm{I}+$ cyclopentane & $6.61 \times 10^{-12} \exp (-503 / T)$ & (Furuyama et al., 1970b) & $\mathrm{HI}+\mathrm{HC} 5 \mathrm{R} \rightarrow \mathrm{I}+\mathrm{HC} 5$ \\
\hline 112 & $\mathrm{HI}+\mathrm{HC}(\mathrm{O}) \rightarrow \mathrm{I}+\mathrm{HC}(\mathrm{O}) \mathrm{H}$ & $3.09 \times 10^{-12} \exp (-98 / T)$ & (Becerra et al., 1997) & $\mathrm{HI}+\mathrm{HCHOR} \rightarrow \mathrm{I}+\mathrm{HCHO}$ \\
\hline 113 & $\mathrm{HI}+\mathrm{CH}_{3} \rightarrow \mathrm{I}+\mathrm{CH}_{4}$ & $4.50 \times 10^{-12} \exp (144 / T)$ & (Seetula et al., 1990) & $\mathrm{HI}+\mathrm{MR} \rightarrow \mathrm{I}+\mathrm{CH} 4$ \\
\hline 114 & $\mathrm{HI}+$ cyclopentenyl $\rightarrow \mathrm{I}+$ cyclopentene & $1.66 \times 10^{-12} \exp (-503 / T)$ & (Furuyama et al., 1970a) & $\mathrm{HI}+\mathrm{OLIR} \rightarrow \mathrm{I}+\mathrm{OLI}$ \\
\hline 115 & $\mathrm{HI}+\mathrm{CH}_{2}=\mathrm{CHCH}_{2} \rightarrow \mathrm{I}+\mathrm{CH}_{2}=\mathrm{CHCH}_{3}$ & $1.69 \times 10^{-11} \exp (-2466 / T)$ & (Rossi and Golden, 1979) & $\mathrm{HI}+\mathrm{OLTR} \rightarrow \mathrm{I}+\mathrm{OLT}$ \\
\hline 116 & $\mathrm{HI}+$ benzyl $\rightarrow \mathrm{I}+$ toluene & $2.96 \times 10^{-14} \exp (-754 / T)$ & (Walsh et al., 1966) & $\mathrm{HI}+\mathrm{TOLR} \rightarrow \mathrm{I}+\mathrm{TOL}$ \\
\hline 117 & $\mathrm{I}+$ phenyl $\rightarrow$ iodobenzene & $2.49 \times 10^{-11}$ & (Robaugh and Tsang, 1986) & $\mathrm{I}+\mathrm{CSLR} \rightarrow$ I_CSL \\
\hline 118 & $\mathrm{I}+\mathrm{C}_{2} \mathrm{H}_{3} \rightarrow \mathrm{CH}_{2}=\mathrm{CHI}$ & $7.31 \times 10^{-12} \exp (-503 / T)$ & (Lifshitz et al., 2008) & I + ETER $\rightarrow$ I_ETE \\
\hline
\end{tabular}




\begin{tabular}{|c|c|c|c|c|}
\hline$\#$ & Reaction & $\begin{array}{c}\text { Rate coefficient } \\
\left(\mathrm{s}^{-1}\right) \text { or }\left(\mathrm{cm}^{3} \text { molecule } \mathrm{s}^{-1}\right) \\
\text { or }\left(\mathrm{cm}^{6} \text { molecule } \mathrm{s}^{-2} \mathrm{~s}^{-1}\right)\end{array}$ & Reference & IRACM reaction \\
\hline 119 & $\mathrm{I}+\mathrm{CH}_{3} \mathrm{CH}_{2} \rightarrow \mathrm{C}_{2} \mathrm{H}_{5} \mathrm{I}$ & $1.16 \times 10^{-11}$ & (Hunter and Kristjansson, 1982) & I + ETHR $\rightarrow$ I_ETH \\
\hline 120 & $\mathrm{I}+\mathrm{CH}_{3} \mathrm{O} \rightarrow \mathrm{HI}+\mathrm{HC}(\mathrm{O}) \mathrm{H}$ & $8.50 \times 10^{-11}$ & (Shah et al., 2001) & $\mathrm{I}+\mathrm{HC} 3 \mathrm{R} \rightarrow \mathrm{HI}+\mathrm{HCHO}$ \\
\hline 121 & $\mathrm{I}+\mathrm{CH}_{3} \rightarrow \mathrm{CH}_{3} \mathrm{I}$ & $9.96 \times 10^{-12}$ & (Hunter and Kristjansson, 1982) & $\mathrm{I}+\mathrm{MR} \rightarrow \mathrm{I} \_\mathrm{CH} 4$ \\
\hline 122 & $\mathrm{I}+\mathrm{CH}_{2}=\mathrm{CHCH}_{2} \rightarrow \mathrm{CH}_{2}=\mathrm{CHCH}_{2} \mathrm{I}$ & $1.60 \times 10^{-10}$ & (Jenkin et al., 1993) & I + OLTR $\rightarrow$ I_OLT \\
\hline 123 & I + benzyl $\rightarrow$ iodomethylbenzene & $8.32 \times 10^{-11}$ & \multicolumn{2}{|c|}{ (Mueller-Markgraf and Troe, 1988) I + TOLR $\rightarrow$ I_TOL } \\
\hline 124 & $\mathrm{I}_{2}+\mathrm{CH}_{3} \mathrm{C}(\mathrm{O}) \rightarrow \mathrm{I}+\mathrm{CH}_{3} \mathrm{C}(\mathrm{O}) \mathrm{I}$ & $6.61 \times 10^{-12}$ & (O'Neal and Benson, 1962a) & $\mathrm{I} 2+$ ALDR $\rightarrow \mathrm{I}+\mathrm{I} \_\mathrm{ALD}$ \\
\hline 125 & $\mathrm{I}_{2}+$ phenyl $\rightarrow \mathrm{I}+$ iodobenzene & $9.13 \times 10^{-12}$ & (Rodgers et al., 1967) & $\mathrm{I} 2+\mathrm{CSLR} \rightarrow \mathrm{I}+\mathrm{I} \_\mathrm{CSL}$ \\
\hline 126 & $\mathrm{I}_{2}+$ cyclopentadienyl $\rightarrow \mathrm{I}+$ iodocyclopentadiene & $2.09 \times 10^{-13}$ & (Furuyama et al., 1971) & I $2+$ DIENR $\rightarrow$ I + I_DIEN \\
\hline 127 & $\mathrm{I}_{2}+\mathrm{CH}_{3} \mathrm{CH}_{2} \rightarrow \mathrm{I}+\mathrm{CH}_{3} \mathrm{CH}_{2} \mathrm{I}$ & $5.00 \times 10^{-11}$ & (Hayes and Strong, 1986) & $\mathrm{I} 2+\mathrm{ETHR} \rightarrow \mathrm{I}+\mathrm{I} \_\mathrm{ETH}$ \\
\hline 128 & $\mathrm{I}_{2}+\mathrm{i}-\mathrm{C}_{3} \mathrm{H}_{7} \rightarrow \mathrm{I}+\mathrm{i}-\mathrm{C}_{3} \mathrm{H}_{7} \mathrm{I}$ & $3.30 \times 10^{-11}$ & (Christie and Frost, 1965) & $\mathrm{I} 2+\mathrm{HC} 3 \mathrm{R} \rightarrow \mathrm{I}+\mathrm{I} \_\mathrm{HC} 3$ \\
\hline 129 & $\mathrm{I}_{2}+\mathrm{n}-\mathrm{C}_{3} \mathrm{H}_{7} \mathrm{I} \rightarrow \mathrm{I}+\mathrm{n}-\mathrm{C}_{3} \mathrm{H}_{7} \mathrm{I}$ & $5.00 \times 10^{-11}$ & (Hayes and Strong, 1986) & $\mathrm{I} 2+\mathrm{HC} 3 \mathrm{R} \rightarrow \mathrm{I}+\mathrm{I} \_\mathrm{HC} 3$ \\
\hline 130 & $\mathrm{I}_{2}+$ cyclopentyl $\rightarrow \mathrm{I}+$ iodocyclopentane & $2.09 \times 10^{-11}$ & (Furuyama et al., 1970b) & $\mathrm{I} 2+\mathrm{HC} 5 \mathrm{R} \rightarrow \mathrm{I}+\mathrm{I} \_\mathrm{HC} 5$ \\
\hline 131 & $\mathrm{I}_{2}+\mathrm{CH}_{3} \rightarrow \mathrm{I}+\mathrm{CH}_{3} \mathrm{I}$ & $5.81 \times 10^{-12}$ & (Hunter and Kristjansson, 1982) & $\mathrm{I} 2+\mathrm{MR} \rightarrow \mathrm{I}+\mathrm{I} \_\mathrm{CH} 43$ \\
\hline 132 & $\mathrm{I}_{2}+$ cyclopentenyl $\rightarrow \mathrm{I}+$ iodocyclopentene & $5.25 \times 10^{-12}$ & (Furuyama et al., 1970a) & $\mathrm{I} 2+$ OLIR $\rightarrow$ I + I_OLI \\
\hline 133 & $\mathrm{I}_{2}+\mathrm{CH}_{2}=\mathrm{CHCH}_{2} \rightarrow \mathrm{CH}_{2}=\mathrm{CHCH}_{2} \mathrm{I}+\mathrm{I}$ & $1.20 \times 10^{-11}$ & (Jenkin et al., 1993) & $\mathrm{I} 2+$ OLTR $\rightarrow$ I + I_OLT \\
\hline 134 & $\mathrm{I}_{2}+$ benzyl $\rightarrow \mathrm{I}+$ iodomethylbenzene & $9.33 \times 10^{-14}$ & (Walsh et al., 1966) & $\mathrm{I} 2+\mathrm{TOLR} \rightarrow \mathrm{I}+\mathrm{I} \_\mathrm{TOL}$ \\
\hline 135 & $\mathrm{IO}+\mathrm{CH}_{3} \mathrm{O} \rightarrow \mathrm{HOI}+\mathrm{HC}(\mathrm{O}) \mathrm{H}$ & $4.00 \times 10^{-11}$ & (Shah et al., 2001) & $\mathrm{IO}+\mathrm{HC} 3 \mathrm{R} \rightarrow \mathrm{HIO}+\mathrm{HCHO}$ \\
\hline 136 & $\mathrm{CH}_{3} \mathrm{I}+\mathrm{CH}_{3} \rightarrow \mathrm{CH}_{2} \mathrm{I}+\mathrm{CH}_{4}$ & $1.05 \times 10^{-12} \exp (-6 / T)$ & (Saito et al., 1980) & I_CH4 + MR $\rightarrow$ I_MR + CH4 \\
\hline 137 & iodobenzene + phenyl $\rightarrow$ products & $2.00 \times 10^{-11}$ & (Alecu et al., 2007) & I_CSL + CSLR $\rightarrow$ PROD \\
\hline 138 & $\mathrm{CH}_{3} \mathrm{CH}_{2} \mathrm{I}+\mathrm{CH}_{3} \rightarrow \mathrm{CH}_{3} \mathrm{I}+\mathrm{CH}_{3} \mathrm{CH}_{2}$ & $1.32 \times 10^{-12} \exp (-3 / T)$ & (Sidebottom and Treacy, 1984) & I_ETH + MR $\rightarrow$ I_CH4 + ETHR \\
\hline 139 & $\mathrm{CH}_{2} \mathrm{I}+\mathrm{CH}_{3} \rightarrow \mathrm{CH}_{3} \mathrm{CH}_{2} \mathrm{I}$ & $5.23 \times 10^{-11}$ & (Saito et al., 1980) & I_MR + MR $\rightarrow$ I_ETH \\
\hline
\end{tabular}

${ }^{a}$ Branching ratio from (Cotter et al., 2003). 
Appendix A.2: Inorganic iodine gas-phase mechanism

\begin{tabular}{|c|c|c|c|}
\hline$\#$ & Reaction & $\begin{array}{c}\text { Rate coefficient } \mathrm{t}^{\mathrm{a}} \\
\left(\mathrm{s}^{-1}\right) \text { or }\left(\mathrm{cm}^{3} \text { molecule } \mathrm{e}^{-1} \mathrm{~s}^{-1}\right) \\
\text { or }\left(\mathrm{cm}^{6} \text { molecule }^{-2} \mathrm{~s}^{-1}\right)\end{array}$ & Reference \\
\hline 1 & $\mathrm{HI}+\mathrm{H} \rightarrow \mathrm{I}+\mathrm{H}_{2}$ & $6.08 \times 10^{-11} \exp (-361 / T)$ & (Umemoto et al., 1988) \\
\hline 2 & $\mathrm{HI}+\mathrm{HI} \rightarrow \mathrm{I}_{2}+\mathrm{H}_{2}$ & $4.25 \times 10^{-11} \exp (-22021 / T)$ & (Baulch et al., 1981) \\
\hline 3 & $\mathrm{HI}+\mathrm{OH} \rightarrow \mathrm{HOI}+\mathrm{H}$ & $1.49 \times 10^{-18} T^{2.28} \exp (-12394 / T)$ & (Xerri et al., 2012) \\
\hline 4 & $\mathrm{HI}+\mathrm{OH} \rightarrow \mathrm{I}+\mathrm{H}_{2} \mathrm{O}$ & $1.60 \times 10^{-11} \exp (440 / T)$ & (Atkinson et al., 2007) \\
\hline 5 & $\mathrm{HI}+\mathrm{I} \rightarrow \mathrm{I}_{2}+\mathrm{H}$ & $9.70 \times 10^{-16} T^{1.72} \exp (-17930 / T)$ & (Canneaux et al., 2010) \\
\hline 6 & $\mathrm{HI}+\mathrm{IO} \rightarrow \mathrm{HOI}+\mathrm{I}$ & $3.01 \times 10^{-19} T^{2.36} \exp (-673 / T)$ & (Hammaecher et al., 2011) \\
\hline 7 & $\mathrm{HI}+\mathrm{NO}_{2} \rightarrow \mathrm{I}+\mathrm{HONO}$ & $3.60 \times 10^{-14} \exp (-3249 / T)$ & (Diao and Chu, 2001) \\
\hline 8 & $\mathrm{HI}+\mathrm{NO}_{3} \rightarrow \mathrm{I}+\mathrm{HNO}_{3}$ & $1.30 \times 10^{-12} \exp (-1829 / T)$ & (Lancar et al., 1991) \\
\hline 9 & $\mathrm{HI}+\mathrm{O}\left({ }^{3} \mathrm{P}\right) \rightarrow \mathrm{OH}+\mathrm{I}$ & $6.68 \times 10^{-11} \exp (-999 / T)$ & (Singleton and Cvetanović, 1978) \\
\hline 10 & $\mathrm{HI}+\mathrm{O}_{2} \rightarrow \mathrm{I}+\mathrm{HO}_{2}$ & $3.30 \times 10^{-12} \exp (-11131 / T)$ & (Shum and Benson, 1983) \\
\hline 11 & $\mathrm{HOI}+\mathrm{H} \rightarrow \mathrm{HI}+\mathrm{OH}$ & $1.89 \times 10^{-15} T^{1.49} \exp (1155 / T)$ & (Xerri et al., 2012) \\
\hline 12 & $\mathrm{HOI}+\mathrm{H} \rightarrow \mathrm{I}+\mathrm{H}_{2} \mathrm{O}$ & $9.96 \times 10^{-16} T^{1.55} \exp (-1576 / T)$ & (Xerri et al., 2012) \\
\hline 13 & $\mathrm{HOI}+\mathrm{H} \rightarrow \mathrm{IO}+\mathrm{H}_{2}$ & $1.40 \times 10^{-23} T^{3.66} \exp (-2166 / T)$ & (Hammaecher et al., 2011) \\
\hline 14 & $\mathrm{HOI}+\mathrm{HNO}_{2} \rightarrow \mathrm{INO}_{2}+\mathrm{H}_{2} \mathrm{O}$ & $8.30 \times 10^{-16}$ & (Vickis, 1989) \\
\hline 15 & $\mathrm{HOI}+\mathrm{OH} \rightarrow \mathrm{IO}+\mathrm{H}_{2} \mathrm{O}$ & $3.60 \times 10^{-27} T^{4.41} \exp (2395 / T)$ & (Hammaecher et al., 2011) \\
\hline 16 & $\mathrm{HOI}+\mathrm{I} \rightarrow \mathrm{HI}+\mathrm{IO}$ & $3.80 \times 10^{-18} T^{2.29} \exp (-13237 / T)$ & (Hammaecher et al., 2011) \\
\hline 17 & $\mathrm{HOI}+\mathrm{I} \rightarrow \mathrm{I}_{2}+\mathrm{OH}$ & $2.61 \times 10^{-16} T^{1.72} \exp (-5283 / T)$ & (Xerri et al., 2012) \\
\hline 18 & $\mathrm{HOI}+\mathrm{O}\left({ }^{3} \mathrm{P}\right) \rightarrow \mathrm{IO}+\mathrm{OH}$ & $2.81 \times 10^{-27} T^{4.65} \exp (-300 / T)$ & (Hammaecher et al., 2011) \\
\hline 19 & $\mathrm{HOI}+\mathrm{O}_{2} \rightarrow \mathrm{IO}+\mathrm{HO}_{2}$ & 0.06 & (Jenkin et al., 1991) \\
\hline 20 & $\mathrm{I}+\mathrm{H}(+\mathrm{M}) \rightarrow \mathrm{HI}(+\mathrm{M})$ & $1.3 \times 10^{-31}(T / 298)^{1.87}$ & (Lifshitz et al., 2008) \\
\hline 21 & $\mathrm{I}+\mathrm{H}_{2} \rightarrow \mathrm{HI}+\mathrm{H}$ & $4.52 \times 10^{-10} \exp (-17087 / T)$ & (Michael et al., 2000) \\
\hline
\end{tabular}




\begin{tabular}{|c|c|c|c|}
\hline \# & Reaction & $\begin{array}{c}\text { Rate coefficient }{ }^{\mathrm{a}} \\
\left(\mathrm{s}^{-1}\right) \text { or }\left(\mathrm{cm}^{3} \text { molecule } \mathrm{e}^{-1} \mathrm{~s}^{-1}\right) \\
\text { or }\left(\mathrm{cm}^{6} \text { molecule }^{-2} \mathrm{~s}^{-1}\right)\end{array}$ & Reference \\
\hline 22 & $\mathrm{I}+\mathrm{H}_{2} \mathrm{O} \rightarrow \mathrm{HI}+\mathrm{OH}$ & $5.20 \times 10^{-17} T^{2.26} \exp (-21780 / T)$ & (Canneaux et al., 2010) \\
\hline 23 & $\mathrm{I}+\mathrm{H}_{2} \mathrm{O} \rightarrow \mathrm{HOI}+\mathrm{H}$ & $4.20 \times 10^{-18} T^{2.48} \exp (-36462 / T)$ & (Xerri et al., 2012) \\
\hline 24 & $\mathrm{I}+\mathrm{H}_{2} \mathrm{O}_{2} \rightarrow \mathrm{HOI}+\mathrm{OH}$ & $3.11 \times 10^{-20} T^{2.95} \exp (-7244 / T)$ & (Fortin et al., 2018) \\
\hline 25 & $\mathrm{I}+\mathrm{OH} \rightarrow \mathrm{HI}+\mathrm{O}\left({ }^{3} \mathrm{P}\right)$ & $4.70 \times 10^{-20} T^{1.70} \exp (-14922 / T)$ & (Canneaux et al., 2010) \\
\hline 26 & $\mathrm{I}+\mathrm{OH} \rightarrow \mathrm{IO}+\mathrm{H}$ & $2.51 \times 10^{-10} \exp (-32250 / T)$ & (Garrett and Truhlar, 1979) \\
\hline 27 & $\mathrm{I}+\mathrm{HO}_{2} \rightarrow \mathrm{HI}+\mathrm{O}_{2}$ & $1.47 \times 10^{-11} \exp (-1155 / T)$ & (Jenkin et al., 1990) \\
\hline 28 & $\mathrm{I}+\mathrm{I}(+\mathrm{M}) \rightarrow \mathrm{I}_{2}(+\mathrm{M})$ & $k_{0}=1.20 \times 10^{-32}, k_{\infty}=2.99 \times 10^{-11}, F_{\mathrm{c}}=1$ & (Hippler et al., 1973) \\
\hline 29 & $\mathrm{I}+\mathrm{I}_{2} \mathrm{O} \rightarrow \mathrm{I}_{2}+\mathrm{IO}$ & $3.82 \times 10^{-11}$ & (Hippler et al., 1973) \\
\hline 30 & $\mathrm{I}+\mathrm{INO} \rightarrow \mathrm{I}_{2}+\mathrm{NO}$ & $1.66 \times 10^{-10}$ & (Basco and Hunt, 1978) \\
\hline 31 & $\mathrm{I}+\mathrm{INO}_{2} \rightarrow \mathrm{I}_{2}+\mathrm{NO}_{2}$ & $8.32 \times 10^{-11}$ & (van den Bergh and Troe, 1976) \\
\hline 32 & $\mathrm{I}+\mathrm{IO} \rightarrow \mathrm{I}_{2} \mathrm{O}$ & $1.69 \times 10^{-10}$ & (Bloss et al., 2001) \\
\hline 33 & $\mathrm{I}+\mathrm{IONO}_{2} \rightarrow \mathrm{I}_{2}+\mathrm{NO}_{3}$ & $9.10 \times 10^{-11} \exp (-145 / T)$ & (Kaltsoyannis and Plane, 2008) \\
\hline 34 & $\mathrm{I}+\mathrm{NO}(+\mathrm{M}) \rightarrow \mathrm{INO}(+\mathrm{M})$ & $k_{0}=1.80 \times 10^{-32}(T / 300)^{-1}, k_{\infty}=1.70 \times 10^{-11}, F_{\mathrm{c}}=0.6$ & (van den Bergh and Troe, 1976) \\
\hline 35 & $\mathrm{I}+\mathrm{NO}_{2}(+\mathrm{M}) \rightarrow \mathrm{INO}_{2}(+\mathrm{M})$ & $k_{0}=3.20 \times 10^{-31}, k_{\infty}=1.10 \times 10^{-10}, F_{\mathrm{c}}=0.48$ & (Tucceri et al., 2005) \\
\hline 36 & $\mathrm{I}+\mathrm{NO}_{3} \rightarrow \mathrm{IO}+\mathrm{NO}_{2}$ & $1.00 \times 10^{-10}$ & (Dillon et al., 2008) \\
\hline 37 & $\mathrm{I}+\mathrm{O}_{3} \rightarrow \mathrm{IO}+\mathrm{O}_{2}$ & $2.10 \times 10^{-11} \exp (-830 / T)$ & (Atkinson et al., 2007) \\
\hline 38 & $\mathrm{I}_{2}+\mathrm{H} \rightarrow \mathrm{HI}+\mathrm{I}$ & $6.59 \times 10^{-10} \exp (-20 / T)$ & (Lorenz et al., 1979) \\
\hline 39 & $\mathrm{I}_{2}+\mathrm{H}_{2} \rightarrow \mathrm{HI}+\mathrm{HI}$ & $1.73 \times 10^{-10} \exp (-20457 / T)$ & (Sullivan, 1959) \\
\hline 40 & $\mathrm{I}_{2}+\mathrm{OH} \rightarrow \mathrm{HOI}+\mathrm{I}$ & $1.97 \times 10^{-10} \exp (-20 / T)$ & (IUPAC, 2007) \\
\hline 41 & $\mathrm{I}_{2}+\mathrm{NO}_{3} \rightarrow \mathrm{I}+\mathrm{IONO}_{2}$ & $1.50 \times 10^{-12}$ & (Chambers et al., 1992) \\
\hline 42 & $\mathrm{I}_{2}+\mathrm{O} \rightarrow \mathrm{I}+\mathrm{IO}$ & $1.20 \times 10^{-10}$ & (Tucceri et al., 2005) \\
\hline
\end{tabular}




\begin{tabular}{|c|c|c|c|}
\hline \# & Reaction & $\begin{array}{c}\text { Rate coefficient }{ }^{\mathrm{a}} \\
\left(\mathrm{s}^{-1}\right) \text { or }\left(\mathrm{cm}^{3} \text { molecule }{ }^{-1} \mathrm{~s}^{-1}\right) \\
\text { or }\left(\mathrm{cm}^{6} \text { molecule } \mathrm{s}^{-2}\right)\end{array}$ & Reference \\
\hline \multirow{2}{*}{43} & $\mathrm{I}_{2}+\mathrm{O}_{3} \rightarrow \mathrm{IO}+\mathrm{OIO}$ & $7.74 \times 10^{-14} \exp (-2888 / T) \times 0.5^{b}$ & \multirow{2}{*}{ (Vikis and MacFarlane, 1985) } \\
\hline & $\rightarrow \mathrm{I}+\mathrm{IO}+\mathrm{O}_{2}$ & $7.74 \times 10^{-14} \exp (-2888 / T) \times 0.5^{b}$ & \\
\hline 44 & $\mathrm{I}_{2} \rightarrow \mathrm{I}+\mathrm{I}$ & $3.32 \times 10^{-11} \exp (-7906 / T)$ & (O'Neal and Benson, 1962b) \\
\hline 45 & $\mathrm{I}_{2} \mathrm{O}_{2}+\mathrm{O}_{3} \rightarrow \mathrm{I}_{2} \mathrm{O}_{3}+\mathrm{O}_{2}$ & $5.0 \times 10^{-16}$ & (Gómez Martin et al., 2013) \\
\hline 46 & $\mathrm{I}_{2} \mathrm{O}_{2} \rightarrow \mathrm{I}+\mathrm{OIO}$ & $1.2 \times 10^{-3}$ & (Gómez Martin et al., 2013) \\
\hline 47 & $\mathrm{I}_{2} \mathrm{O}_{2} \rightarrow \mathrm{IO}+\mathrm{IO}$ & $4.0 \times 10^{-2}$ & (Sommariva et al., 2012) \\
\hline 48 & $\mathrm{I}_{2} \mathrm{O}_{3}+\mathrm{I}_{2} \mathrm{O}_{3} \rightarrow \mathrm{I}_{4} \mathrm{O}_{6}$ & $1.40 \times 10^{-12}$ & (Gómez Martin et al., 2013) \\
\hline 49 & $\mathrm{I}_{2} \mathrm{O}_{3}+\mathrm{I}_{2} \mathrm{O}_{4} \rightarrow \mathrm{I}_{4} \mathrm{O}_{7}$ & $2.70 \times 10^{-11}$ & (Gómez Martin et al., 2013) \\
\hline 50 & $\mathrm{I}_{2} \mathrm{O}_{3}+\mathrm{I}_{2} \mathrm{O}_{5} \rightarrow \mathrm{I}_{4} \mathrm{O}_{8}$ & $1.90 \times 10^{-11}$ & (Gómez Martin et al., 2013) \\
\hline 51 & $\mathrm{I}_{2} \mathrm{O}_{3}+\mathrm{O}_{3} \rightarrow \mathrm{I}_{2} \mathrm{O}_{4}+\mathrm{O}_{2}$ & $1.00 \times 10^{-12}$ & (Sommariva et al., 2012) \\
\hline 52 & $\mathrm{I}_{2} \mathrm{O}_{4}+\mathrm{I}_{2} \mathrm{O}_{4} \rightarrow \mathrm{I}_{4} \mathrm{O}_{8}$ & $2.7 \times 10^{-10}$ & (Gómez Martin et al., 2013) \\
\hline 53 & $\mathrm{I}_{2} \mathrm{O}_{4}+\mathrm{I}_{2} \mathrm{O}_{5} \rightarrow \mathrm{I}_{4} \mathrm{O}_{9}$ & $1.2 \times 10^{-11}$ & (Gómez Martin et al., 2013) \\
\hline 54 & $\mathrm{I}_{2} \mathrm{O}_{4}+\mathrm{IO} \rightarrow \mathrm{I}_{3} \mathrm{O}_{5}$ & $1.5 \times 10^{-11}$ & (Gómez Martin et al., 2013) \\
\hline 55 & $\mathrm{I}_{2} \mathrm{O}_{4}+\mathrm{O}_{3} \rightarrow \mathrm{I}_{2} \mathrm{O}_{5}+\mathrm{O}_{2}$ & $1.0 \times 10^{-12}$ & (Sommariva et al., 2012) \\
\hline 56 & $\mathrm{I}_{2} \mathrm{O}_{4}+\mathrm{OIO} \rightarrow \mathrm{I}_{3} \mathrm{O}_{6}$ & $1.5 \times 10^{-10}$ & (Gómez Martin et al., 2013) \\
\hline 57 & $\mathrm{I}_{2} \mathrm{O}_{4} \rightarrow \mathrm{OIO}+\mathrm{OIO}$ & $1.20 \times 10^{-2}$ & (Gómez Martin et al., 2013) \\
\hline 58 & $\mathrm{I}_{2} \mathrm{O}_{5}+\mathrm{I}_{2} \mathrm{O}_{5} \rightarrow \mathrm{I}_{4} \mathrm{O}_{10}$ & $7.0 \times 10^{-11}$ & (Gómez Martin et al., 2013) \\
\hline 59 & $\mathrm{I}_{2} \mathrm{O}_{5}+\mathrm{OIO} \rightarrow \mathrm{I}_{3} \mathrm{O}_{7}$ & $1.0 \times 10^{-13}$ & (Gómez Martin et al., 2013) \\
\hline 60 & $\mathrm{I}_{3} \mathrm{O}_{5} \rightarrow \mathrm{I}_{2} \mathrm{O}_{4}+\mathrm{IO}$ & $6.2 \times 10^{4}$ & (Gómez Martin et al., 2013) \\
\hline 61 & $\mathrm{I}_{3} \mathrm{O}_{6} \rightarrow \mathrm{I}_{2} \mathrm{O}_{4}+\mathrm{OIO}$ & $3.5 \times 10^{4}$ & (Gómez Martin et al., 2013) \\
\hline 62 & $\mathrm{I}_{3} \mathrm{O}_{7} \rightarrow \mathrm{I}_{2} \mathrm{O}_{5}+\mathrm{OIO}$ & $3.7 \times 10^{7}$ & (Gómez Martin et al., 2013) \\
\hline 63 & $\mathrm{I}_{4} \mathrm{O}_{10} \rightarrow \mathrm{I}_{2} \mathrm{O}_{5}+\mathrm{I}_{2} \mathrm{O}_{5}$ & $1.9 \times 10^{3}$ & (Gómez Martin et al., 2013) \\
\hline
\end{tabular}




\begin{tabular}{|c|c|c|c|}
\hline \# & Reaction & $\begin{array}{c}\text { Rate coefficient }^{\mathrm{a}} \\
\left(\mathrm{s}^{-1}\right) \text { or }\left(\mathrm{cm}^{3} \text { molecule } \mathrm{e}^{-1} \mathrm{~s}^{-1}\right) \\
\text { or }\left(\mathrm{cm}^{6} \text { molecule }^{-2} \mathrm{~s}^{-1}\right)\end{array}$ & Reference \\
\hline 64 & $\mathrm{I}_{4} \mathrm{O}_{6} \rightarrow \mathrm{I}_{2} \mathrm{O}_{3}+\mathrm{I}_{2} \mathrm{O}_{3}$ & $7.5 \times 10^{5}$ & (Gómez Martin et al., 2013) \\
\hline 65 & $\mathrm{I}_{4} \mathrm{O}_{7} \rightarrow \mathrm{I}_{2} \mathrm{O}_{3}+\mathrm{I}_{2} \mathrm{O}_{4}$ & $1.6 \times 10^{4}$ & (Gómez Martin et al., 2013) \\
\hline 66 & $\mathrm{I}_{4} \mathrm{O}_{9} \rightarrow \mathrm{I}_{2} \mathrm{O}_{3}+\mathrm{I}_{2} \mathrm{O}_{5}$ & $2.8 \times 10^{3}$ & (Gómez Martin et al., 2013) \\
\hline 67 & $\mathrm{I}_{4} \mathrm{O}_{9} \rightarrow \mathrm{I}_{2} \mathrm{O}_{4}+\mathrm{I}_{2} \mathrm{O}_{5}$ & 8.7 & (Gómez Martin et al., 2013) \\
\hline 68 & $\mathrm{INO}+\mathrm{INO} \rightarrow \mathrm{I}_{2}+2 \mathrm{NO}$ & $2.9 \times 10^{-12} \exp (-1323 / T)$ & (Basco and Hunt, 1978) \\
\hline 69 & $\mathrm{INO}(+\mathrm{M}) \rightarrow \mathrm{I}+\mathrm{NO}$ & $5.83 \times 10^{-13}$ & (Basco and Hunt, 1978) \\
\hline 70 & $\mathrm{INO}_{2}+\mathrm{INO}_{2} \rightarrow \mathrm{I}_{2}+2 \mathrm{NO}_{2}$ & $4.70 \times 10^{-13} \exp (-1672 / T)$ & (Gawler et al., 2003) \\
\hline 71 & $\mathrm{INO}_{2} \rightarrow \mathrm{I}+\mathrm{NO}_{2}$ & $9.93 \times 10^{17} \exp (-11865 / T)$ & (McFiggans et al., 2010) \\
\hline 72 & $\mathrm{IO}+\mathrm{H}_{2} \rightarrow \mathrm{HOI}+\mathrm{H}$ & $1.1 \times 10^{-24} T^{4.00} \exp (-5270 / T)$ & (Hammaecher et al., 2011) \\
\hline 73 & $\mathrm{IO}+\mathrm{H}_{2} \mathrm{O} \rightarrow \mathrm{HOI}+\mathrm{OH}$ & $3.2 \times 10^{-27} T^{4.54} \exp (-7870 / T)$ & (Hammaecher et al., 2011) \\
\hline 74 & $\mathrm{IO}+\mathrm{OH} \rightarrow \mathrm{HO}_{2}+\mathrm{I}$ & $1.0 \times 10^{-10}$ & (Bösch et al., 2003) \\
\hline 75 & $\mathrm{IO}+\mathrm{HO}_{2} \rightarrow \mathrm{HOI}+\mathrm{O}_{2}$ & $1.40 \times 10^{-11} \exp (540 / T)$ & (Atkinson et al., 2007) \\
\hline \multirow{4}{*}{76} & $\mathrm{IO}+\mathrm{IO} \rightarrow \mathrm{I}_{2}+\mathrm{O}_{2}$ & $5.40 \times 10^{-11} \exp (180 / T) \times 0.05^{\mathrm{c}}$ & \multirow{4}{*}{ (Atkinson et al., 2007) } \\
\hline & $\rightarrow 2 \mathrm{I}+\mathrm{O}_{2}$ & $5.40 \times 10^{-11} \exp (180 / T) \times 0.09^{c}$ & \\
\hline & $\rightarrow \mathrm{I}+\mathrm{OIO}$ & $5.40 \times 10^{-11} \exp (180 / T) \times 0.45^{\mathrm{c}}$ & \\
\hline & $\rightarrow \mathrm{I}_{2} \mathrm{O}_{2}$ & $5.40 \times 10^{-11} \exp (180 / T) \times 0.44^{\mathrm{c}}$ & \\
\hline 77 & $\mathrm{IO}+\mathrm{NO} \rightarrow \mathrm{I}+\mathrm{NO}_{2}$ & $7.15 \times 10^{-12} \exp (299 / T)$ & (Atkinson et al., 2007) \\
\hline 78 & $\mathrm{IO}+\mathrm{NO}_{2}(+\mathrm{M}) \rightarrow \mathrm{IONO}_{2}(+\mathrm{M})$ & $k_{0}=7.70 \times 10^{-31}(T / 300)^{-5}, k_{\infty}=1.50 \times 10^{-11}, F_{\mathrm{c}}=0.4$ & (Daykin and Wine, 1990) \\
\hline 79 & $\mathrm{IO}+\mathrm{NO}_{3} \rightarrow \mathrm{OIO}+\mathrm{NO}_{2}$ & $6.10 \times 10^{-12}$ & (Dillon et al., 2008) \\
\hline 80 & $\mathrm{IO}+\mathrm{O}\left({ }^{3} \mathrm{P}\right) \rightarrow \mathrm{I}+\mathrm{O}_{2}$ & $1.35 \times 10^{-10}$ & (Canosa-Mas et al., 1999) \\
\hline 81 & $\mathrm{IO}+\mathrm{O}_{3} \rightarrow \mathrm{OIO}+\mathrm{O}_{2}$ & $3.60 \times 10^{-16}$ & (Dillon et al., 2006) \\
\hline 82 & $\mathrm{IO}+\mathrm{O}_{3} \rightarrow \mathrm{I}+2 \mathrm{O}_{2}$ & $1.20 \times 10^{-15}$ & (Larin et al., 1999) \\
\hline
\end{tabular}




\begin{tabular}{|c|c|c|c|}
\hline \# & Reaction & $\begin{array}{c}\text { Rate coefficient }^{\mathrm{a}} \\
\left(\mathrm{s}^{-1}\right) \text { or }\left(\mathrm{cm}^{3} \text { molecule } \mathrm{e}^{-1} \mathrm{~s}^{-1}\right) \\
\text { or }\left(\mathrm{cm}^{6} \text { molecule }^{-2} \mathrm{~s}^{-1}\right)\end{array}$ & Reference \\
\hline 83 & $\mathrm{IO}+\mathrm{OIO} \rightarrow \mathrm{I}_{2} \mathrm{O}_{3}$ & $1.15 \times 10^{-10}$ & (Galvez et al., 2013) \\
\hline 84 & $\mathrm{IONO}_{2} \rightarrow \mathrm{IO}+\mathrm{NO}$ & $2.10 \times 10^{15} \exp (-13718 / T)$ & (Kaltsoyannis and Plane, 2008) \\
\hline 85 & $\mathrm{OIO}+\mathrm{OH}(+\mathrm{M}) \rightarrow \mathrm{HOIO}_{2}(+\mathrm{M})$ & $k_{0}=1.50 \times 10^{-27}(T / 300)^{-3.93}, k_{\infty}=5.5 \times 10^{-10} \exp (46 / T), F_{\mathrm{c}}=0.3$ & (Plane et al., 2006) \\
\hline 86 & $\mathrm{OIO}+\mathrm{NO} \rightarrow \mathrm{IO}+\mathrm{NO}_{2}$ & $7.60 \times 10^{-13} \exp (606 / T)$ & (Plane et al., 2006) \\
\hline 87 & $\mathrm{OIO}+\mathrm{OIO} \rightarrow \mathrm{I}_{2} \mathrm{O}_{4}$ & $4.20 \times 10^{-11}$ & (Galvez et al., 2013) \\
\hline 88 & $\mathrm{IO}+\mathrm{CO} \rightarrow \mathrm{I}+\mathrm{CO}_{2}$ & $1.49 \times 10^{-17} T^{1.77} \exp (-5703 / T)$ & (Khanniche et al., 2016b) \\
\hline 89 & $\mathrm{I}_{2} \mathrm{O}_{5}+\mathrm{H}_{2} \mathrm{O} \rightarrow 2 \mathrm{HOIO}_{2}$ & $3.61 \times 10^{-22} T^{2.05} \exp (-3886 / T)$ & (Khanniche et al., 2016a) \\
\hline 90 & $\mathrm{HOI}+\mathrm{NO}_{3} \rightarrow \mathrm{IO}+\mathrm{HNO}_{3}$ & $2.70 \times 10^{-12}(300 / T)^{2.66}$ & (Saiz-Lopez et al., 2016) \\
\hline 91 & $2 \mathrm{HOIO}_{2} \rightarrow \mathrm{I}_{2} \mathrm{O}_{5}+\mathrm{H}_{2} \mathrm{O}$ & $6.73 \times 10^{-27} T^{2.90} \exp (-2948 / T)$ & (Khanniche et al., 2016a) \\
\hline 92 & $\mathrm{HOIO}_{2}+\mathrm{OH} \rightarrow \mathrm{OIO}_{2}+\mathrm{H}_{2} \mathrm{O}$ & $1.76 \times 10^{-22} T^{2.39} \exp (-421 / T)$ & (Khanniche et al., 2017) \\
\hline
\end{tabular}

${ }^{\mathrm{a}}$ Kinetic parameters for use in the Troe equation. ${ }^{\mathrm{b}}$ Branching ratio estimated. ${ }^{\mathrm{c}}$ Branching ratio from (Gómez Martin et al., 2013). 
Appendix A.3: Organic iodine gas-phase photolysis mechanism

\begin{tabular}{|c|c|c|c|c|}
\hline & Reaction & $\mathrm{J}\left(\mathrm{s}^{-1}\right)^{\mathrm{a}}$ & Reference & IRACM reaction \\
\hline 1 & $\mathrm{CH}_{2} \mathrm{I}_{2}+\mathrm{hv} \rightarrow \mathrm{CH}_{2} \mathrm{I}+\mathrm{I}$ & $7.29 \times 10^{-3 \mathrm{~d}}$ & $\begin{array}{l}\text { (Burkholder et al., 2015) }^{\mathrm{b}} \\
\text { (Mössinger et al., 1998) }^{\mathrm{b}} \\
\text { (Roehl et al., 1997) }^{\mathrm{b}}\end{array}$ & I2_CH4 + hv $\rightarrow$ I_MR + I \\
\hline 2 & $\mathrm{CH}_{3} \mathrm{I}+\mathrm{hv} \rightarrow \mathrm{CH}_{3}+\mathrm{I}$ & $5.80 \times 10^{-6 \mathrm{e}}$ & $\begin{array}{l}\text { (Burkholder et al., 2015) }^{\mathrm{b}} \\
\text { (Fahr et al., 1995) }^{\mathrm{b}} \\
\text { (Rattigan et al., 1997) }^{\mathrm{b}} \\
\text { (Jenkin et al., 1993) }^{\mathrm{b}} \\
\text { (Roehl et al., 1997) }^{\mathrm{b}}\end{array}$ & I_CH4 + hv $\rightarrow$ MR + I \\
\hline 3 & $\mathrm{C}_{2} \mathrm{H}_{5} \mathrm{I}+\mathrm{hv} \rightarrow \mathrm{C}_{2} \mathrm{H}_{5}+\mathrm{I}$ & $6.62 \times 10^{-6 f}$ & $\begin{array}{l}\text { (Burkholder et al., 2015) }^{\mathrm{b}} \\
\text { (Rattigan et al., 1997) }^{\mathrm{b}} \\
\text { (Roehl et al., 1997) }^{\mathrm{b}}\end{array}$ & I_ETH + h $v \rightarrow$ ETHR + I \\
\hline 4 & $\mathrm{CH}_{3} \mathrm{CHI}_{2}+\mathrm{hv} \rightarrow$ products & $2.66 \times 10^{-3}$ & $\begin{array}{l}\text { (Burkholder et al., 2015) }^{\mathrm{b}} \\
(\text { Schmitt and Comes, 1980) }\end{array}$ & I2_ETH + hv $\rightarrow$ PROD \\
\hline 5 & $\mathrm{C}_{3} \mathrm{H}_{7} \mathrm{I}+\mathrm{hv} \rightarrow \mathrm{C}_{3} \mathrm{H}_{7}+\mathrm{I}$ & $2.61 \times 10^{-5 g}$ & $\begin{array}{l}\text { (Burkholder et al., 2015) }^{\mathrm{b}} \\
\text { (Roehl et al., 1997) }^{\mathrm{b}}\end{array}$ & $\mathrm{I} \_\mathrm{HC} 3+\mathrm{hv} \rightarrow \mathrm{HC} 3 \mathrm{R}+\mathrm{I}$ \\
\hline 6 & $\mathrm{t}-\mathrm{C}_{4} \mathrm{H}_{9} \mathrm{I}+\mathrm{hv} \rightarrow \mathrm{t}-\mathrm{C}_{4} \mathrm{H}_{9}+\mathrm{I}$ & $1.88 \times 10^{-6}$ & $\begin{array}{l}\text { (Burkholder et al., 2015) } \\
\text { (Phillips et al., 1992) }^{\mathrm{b}} \\
\text { (Kim et al., 1997) }^{\mathrm{c}}\end{array}$ & I_HC3 + hv $\rightarrow$ HC3R + I \\
\hline
\end{tabular}

${ }^{a}$ Peak value at midday: photolysis rates are calculated for clear sky, begin of August, latitude $=51{ }^{\circ} \mathrm{N} .{ }^{\mathrm{b}}$ Cross sections. ${ }^{\mathrm{c}}$ Quantum Yield. ${ }^{\mathrm{d}}$ Cross sections from (Roehl et al., 1997) between $205 \mathrm{~nm}$ and $215 \mathrm{~nm}$, mean of the cross sections from (Roehl et al., 1997) and (Mössinger et al., 1998) between $220 \mathrm{~nm}$ and $380 \mathrm{~nm}$, cross sections from (Mössinger et al., 1998) at $285 \mathrm{~nm}$, evaluation data from (Burkholder et al., 2015). ${ }^{\text {e }}$ Mean of the cross sections from (Jenkin et al., 1993), (Fahr et al., 1995), and (Roehl et al., 1997) between 210 and $230 \mathrm{~nm}$, mean of the cross sections from (Jenkin et al., 1993), (Fahr et al., 1995), (Roehl et al., 1997), and (Rattigan et al., 1997) between 235 and 330 $\mathrm{nm}$, mean of the cross sections from (Fahr et al., 1995), (Rattigan et al., 1997) and (Roehl et al., 1997) between 335 and 350 nm, cross sections from (Rattigan et al., 1997) between 355 and $365 \mathrm{~nm}$, evaluation data from (Burkholder et al., 2015). ${ }^{\mathrm{f}}$ Cross sections from (Roehl et al., 1997) between 205 and $230 \mathrm{~nm}$, mean of the cross sections from (Roehl et al., 1997) and (Rattigan et al., 1997) between 235 and $355 \mathrm{~nm}$, evaluation data from (Burkholder et al., 2015). ${ }^{\mathrm{g}}$ Sum of the photolysis parameters from $\mathrm{CH}_{2} \mathrm{ICH}_{2} \mathrm{CH}_{3}$ and $\mathrm{CH}_{3} \mathrm{CHICH}_{3}$. 
Appendix A.4: Inorganic iodine gas-phase photolysis mechanism

\begin{tabular}{|c|c|c|c|}
\hline$\overline{\#}$ & Reaction & $\mathrm{J}\left(\mathrm{s}^{-1}\right)^{\mathrm{a}}$ & Reference \\
\hline 1 & $\mathrm{I}_{2}+\mathrm{hv} \rightarrow \mathrm{I}+\mathrm{I}$ & $6.24 \times 10^{-2}$ & $\begin{array}{l}\text { (Burkholder et al., 2015) } \\
\text { (Saiz-Lopez et al., 2004) }^{\mathrm{b}} \\
\text { (Brewer and Tellinghuisen, 1972) }^{\mathrm{c}}\end{array}$ \\
\hline 2 & $\mathrm{HI}+\mathrm{h} v \rightarrow \mathrm{I}+\mathrm{H}$ & $8.20 \times 10^{-6}$ & $\begin{array}{l}\text { (Burkholder et al., 2015) }^{\mathrm{b}} \\
\text { (Campuzano-Jost and Crowley, 1999) }^{\mathrm{b}} \\
\text { (Martin and Willard, 1964) }^{\mathrm{c}}\end{array}$ \\
\hline 3 & $\mathrm{HOI}+\mathrm{h} v \rightarrow \mathrm{I}+\mathrm{OH}$ & $3.29 \times 10^{-4 \mathrm{~d}}$ & $\begin{array}{l}\text { (Burkholder et al., 2015) } \\
(\text { Bauer et al., 1998) } \\
\text { (Rowley et al., 1999) }^{\mathrm{b}}\end{array}$ \\
\hline 4 & $\mathrm{INO}+\mathrm{h} v \rightarrow \mathrm{I}+\mathrm{NO}$ & $2.76 \times 10^{--2 \mathrm{e}}$ & $\begin{array}{l}\text { (Burkholder et al., 2015) }^{\mathrm{b}} \\
(\text { Basco and Hunt, 1978) } \\
(\text { Forte et al., 1981) } \\
\text { (van den Bergh and Troe, 1976) }^{\mathrm{b}}\end{array}$ \\
\hline 5 & $\mathrm{INO}_{2}+\mathrm{hv} \rightarrow 0.5 \mathrm{I}+0.5 \mathrm{NO}_{2}+0.5 \mathrm{IO}+0.5 \mathrm{NO}$ & $1.20 \times 10^{-2}$ & $\begin{array}{l}(\text { Burkholder et al., 2015) } \\
(\text { Bröske, 2000) }\end{array}$ \\
\hline 6 & $\mathrm{IONO}_{2}+\mathrm{h} v \rightarrow 0.5 \mathrm{I}+0.5 \mathrm{NO}_{3}+0.5 \mathrm{IO}+0.5 \mathrm{NO}_{2}$ & $9.63 \times 10^{-3}$ & 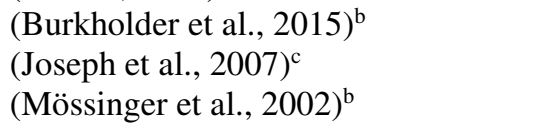 \\
\hline 7 & $\mathrm{I}_{2} \mathrm{O}_{2}+\mathrm{hv} \rightarrow \mathrm{I}+\mathrm{OIO}$ & $1.92 \times 10^{-2 \mathrm{f}}$ & $\left(\right.$ Sherwen et al., 2016a) ${ }^{b}$ \\
\hline 8 & $\mathrm{I}_{2} \mathrm{O}_{3}+\mathrm{hv} \rightarrow \mathrm{IO}+\mathrm{OIO}$ & $1.92 \times 10^{-2 \mathrm{f}}$ & $\left(\right.$ Sherwen et al., 2016a) ${ }^{b}$ \\
\hline 9 & $\mathrm{I}_{2} \mathrm{O}_{4}+\mathrm{hv} \rightarrow \mathrm{OIO}+\mathrm{OIO}$ & $1.92 \times 10^{-2 \mathrm{f}}$ & $\left(\right.$ Sherwen et al., 2016a) ${ }^{b}$ \\
\hline 10 & $\mathrm{IO}+\mathrm{h} v \rightarrow \mathrm{I}+\mathrm{O}\left({ }^{3} \mathrm{P}\right)$ & $3.21 \times 10^{-2}$ & $\begin{array}{l}\text { (Burkholder et al., 2015) }^{\mathrm{b}} \\
\text { (Bloss et al., 2001) }^{\mathrm{b}} \\
\text { (Harwood et al., 1997) }^{\mathrm{b}} \\
\text { (Ingham et al., 2000) }^{\mathrm{c}}\end{array}$ \\
\hline 11 & $\mathrm{OIO}+\mathrm{h} v \rightarrow \mathrm{I}+\mathrm{O}_{2}$ & $2.55 \times 10^{-1}$ & $\begin{array}{l}\text { (Burkholder et al., 2015) }^{\mathrm{b}} \\
\text { (Bloss et al., 2001) }^{\mathrm{b}}\end{array}$ \\
\hline
\end{tabular}

${ }^{a}$ Peak value at midday: photolysis rate are calculated for clear sky, begin of August, latitude $=51^{\circ} \mathrm{N} .{ }^{\mathrm{b}}$ Cross sections. ${ }^{\mathrm{c}}$ Quantum yield. ${ }^{\mathrm{d}}$ Mean of the cross sections from (Bauer et al., 1998) and (Rowley et al., 1999), evaluation data from (Burkholder et al., 2015). ${ }^{\mathrm{e}}$ Mean of the cross sections from (Basco and Hunt, 1978) and (Forte et al., 1981) between 223 and $290 \mathrm{~nm}$, cross sections from (Basco and Hunt, 1978) at 300, 310, $360 \mathrm{~nm}$ and between 370 and $400 \mathrm{~nm}$, mean of the cross sections from (van den Bergh and Troe, 1976), (Basco and Hunt, 1978), and (Forte et al., 1981) for the cross sections greater than $400 \mathrm{~nm}$, evaluation data from (Burkholder et al., 2015). ${ }^{\mathrm{f}}$ Cross sections estimated to be equal to those of $\mathrm{IONO}_{2}$ with a quantum yield equal to 1 . 


\section{References}

Acker, K., Kezele, N., Klasinc, L., Möller, D., Pehnec, G., Šorgo, G., Wieprecht, W., Žužul, S., 2008. Atmospheric $\mathrm{H}_{2} \mathrm{O}_{2}$ measurement and modeling campaign during summer 2004 in Zagreb, Croatia. Atmos. Environ. 42, 2530-2542.

Albaladejo, J., Ballesteros, B., Jiménez, E., Díaz de Mera, Y., Martínez, E., 2003. Gas-phase $\mathrm{OH}$ radical-initiated oxidation of the 3-halopropenes studied by PLP-LIF in the temperature range 228-388 K. Atmos. Environ. 37, 2919-2926.

Alecu, I.M., Gao, Y., Hsieh, P.C., Sand, J.P., Ors, A., McLeod, A., Marshall, P., 2007. Studies of the Kinetics and Thermochemistry of the Forward and Reverse Reaction Cl $+\mathrm{C}_{6} \mathrm{H}_{6}=\mathrm{HCl}+\mathrm{C}_{6} \mathrm{H}_{5}$. J. Phys. Chem. A 111, 3970-3976.

Alfassi, Z.B., Golden, D.M., 1973a. Kinetics of the gas-phase reaction of allyl alcohol with iodine. The heat of formation and stabilization energy of the hydroxyallyl radical. Int. J. Chem. Kinet. 5, 295-303.

Alfassi, Z.B., Golden, D.M., 1973b. Kinetics of the reaction of iodine with acrolein. Bond dissociation energy of the carbonyl carbon-hydrogen bond. J. Am. Chem. Soc. 95, $319-323$.

Atkinson, R., Baulch, D.L., Cox, R.A., Crowley, J.N., Hampson, R.F., Hynes, R.G., Jenkin, M.E., Rossi, M.J., Troe, J., 2007. Evaluated kinetic and photochemical data for atmospheric chemistry: Volume III-gas phase reactions of inorganic halogens. Atmos. Chem. Phys. 7, 981-1191.

Bai, F.-Y., Wang, X., Sun, Y.-Q., Pan, X.-M., 2015. Atmospheric chemistry of alkyl iodides: theoretical studies on the mechanisms and kinetics of $\mathrm{CH}_{3} \mathrm{I} / \mathrm{C}_{2} \mathrm{H}_{5} \mathrm{I}+\mathrm{NO}_{3}$ reactions. RSC Adv. 5, 88087-88095.

Bale, C.S.E., Canosa-Mas, C.E., Shallcross, D.E., Wayne, R.P., 2005. A discharge-flow study of the kinetics of the reactions of $\mathrm{IO}$ with $\mathrm{CH}_{3} \mathrm{O}_{2}$ and $\mathrm{CF}_{3} \mathrm{O}_{2}$. Phys. Chem. Chem. Phys. 7, 2164-2172. 
Basco, N., Hunt, J.E., 1978. The recombination of iodine atoms in the presence of nitric oxide. Int. J. Chem. Kinet. 10, 733-743.

Bauer, D., Ingham, T., Carl, S.A., Moortgat, G.K., Crowley, J.N., 1998. Ultraviolet-Visible Absorption Cross Sections of Gaseous HOI and Its Photolysis at 355 nm. J. Phys. Chem. A 102, 2857-2864.

Baulch, D.L., Duxbury, J., Grant, S.J., Montague, D.C., 1981. Evaluated kinetic data for high temperature reactions. Volume 4. Homogeneous gas phase reactions of halogen- and cyanide- containing species. . J. Phys. Chem. Ref. Data 10, Suppl. 1.

Becerra, R., Carpenter, I.W., Walsh, R., 1997. Time-Resolved Studies of the Kinetics of the Reactions of $\mathrm{CHO}$ with $\mathrm{HI}$ and $\mathrm{HBr}$ : Thermochemistry of the CHO Radical and the C-H Bond Strengths in $\mathrm{CH}_{2} \mathrm{O}$ and CHO. J. Phys. Chem. A 101, 4185-4190.

Benson, S.W., 1961. Reaction of Cyclopropane with Iodine and Some Observations on the Isomerization of Cyclopropane. J. Chem. Phys. 34, 521-526.

Benson, S.W., Bose, A.N., 1962. Kinetics of the Gas-Phase Addition of $\mathrm{HI}$ to $\mathrm{C}_{2} \mathrm{H}_{4}$ and the Pyrolysis of Ethyl Iodide. J. Chem. Phys. 37, 2935-2940.

Benson, S.W., O'Neal, E., 1961. Kinetics of the Reactions of Alkyl Iodides with HI. J. Chem. Phys. 34, 514-520.

Bloss, W.J., Rowley, D.M., Cox, R.A., Jones, R.L., 2001. Kinetics and Products of the IO Self-Reaction. J. Phys. Chem. A 105, 7840-7854.

Bösch, H., Camy-Peyret, C., Chipperfield, M.P., Fitzenberger, R., Harder, H., Platt, U., Pfeilsticker, K., 2003. Upper limits of stratospheric IO and OIO inferred from centerto-limb-darkening-corrected balloon-borne solar occultation visible spectra: Implications for total gaseous iodine and stratospheric ozone. J. Geophys. Res. 108, 4455-4473. 
Bose, A.N., Benson, S.W., 1962. Kinetics of the Reaction of HI with Propylene. J. Chem. Phys. 37, 1081-1084.

Bose, A.N., Benson, S.W., 1963. Kinetics of Addition of HI to Isobutene and Vinyl Chloride. J. Chem. Phys. 38, 878-881.

Bosland, L., Cantrel, L., Girault, N., Clement, B., 2010. Modeling of Iodine Radiochemistry in the ASTEC Severe Accident Code: Description and Application to FPT-2 PHEBUS Test. Nucl. Technol. 171, 88-107.

Brewer, L., Tellinghuisen, J., 1972. Quantum Yield for Unimolecular Dissociation of $I_{2}$ in Visible Absorption. J. Chem. Phys. 56, 3929-3938.

Bröske, R., 2000, Kinetische und spektroskopische Untersuchungen von Nitrylhalogeniden, Halogennitriten und Halogennitraten, Ph-D, Bergische Universität Wuppertal

Buben, S.N., Larin, I.K., Trofimova, E.M., 1995. Kinetics of the reactions of IO radicals with $\mathrm{CHCl}_{2}-\mathrm{CF}_{2} \mathrm{Cl}$ and $\mathrm{HCOOH}$. Kinet. Catal. 36, 745-750.

Burkholder, J.B., Sander, R., Abbatt, J.P.D., Barker, J.R., Huie, R.E., Kolb, C.E., Kurylo, M.J., Orkin, V.L., Wilmouth, D.M., Wine, P.H., 2015. Chemical Kinetics and Photochemical Data for Use in Atmospheric Studies. JPL Publication 15-10, Pasadena.

Butler, E.T., Mandel, E., Polanyi, M., 1945. Rates of pyrolysis and bond energies of substituted organic iodides. Part II. Trans. Faraday Soc. 41, 298-306.

Butler, E.T., Polanyi, M., 1943. Rates of pyrolysis and bond energies of substituted organic iodides (Part I). Trans. Faraday Soc. 39, 19-36.

Campuzano-Jost, P., Crowley, J.N., 1999. Kinetics of the Reaction of OH with HI between 246 and 353 K. J. Phys. Chem. A 103, 2712-2719. 
Canneaux, S., Xerri, B., Louis, F., Cantrel, L., 2010. Theoretical Study of the Gas-Phase Reactions of Iodine Atoms $\left({ }^{2} \mathrm{P}_{3 / 2}\right)$ with $\mathrm{H}_{2}, \mathrm{H}_{2} \mathrm{O}$, HI, and OH. J. Phys. Chem. A 114, 9270-9288.

Canosa-Mas, C.E., Flugge, M.L., Shah, D., Vipond, A., Wayne, R.P., 1999. Kinetics of the Reactions of IO with $\mathrm{HO}_{2}$ and $\mathrm{O}\left({ }^{3} \mathrm{P}\right)$. J. Atmos. Chem. 34, 153-162.

Cantrel, L., Cousin, F., Bosland, L., Chevalier-Jabet, K., Marchetto, C., 2014. ASTEC V2 severe accident integral code: Fission product modelling and validation. Nucl. Eng. Des. 272, 195-206.

Chambers, R.M., Heard, A.C., Wayne, R.P., 1992. Inorganic gas-phase reactions of the nitrate radical: iodine + nitrate radical and iodine atom + nitrate radical. J. Phys. Chem. 96, $3321-3331$.

Chatelard, P., Belon, S., Bosland, L., Carénini, L., Coindreau, O., Cousin, F., Marchetto, C., Nowack, H., Piar, L., Chailan, L., 2016. Main modelling features of the ASTEC V2.1 major version. Ann. Nucl. Energy 93, 83-93.

Chevalier-Jabet, K., Cousin, F., Cantrel, L., Séropian, C., 2014. Source term assessment with ASTEC and associated uncertainty analysis using SUNSET tool. Nucl. Eng. Des. 272, 207-218.

Christie, M.I., Frost, J.S., 1965. Association reactions of alkyl radicals with oxygen and with nitric oxide. Trans. Faraday Soc. 61, 468-473.

Cotter, E.S.N., Canosa-Mas, C.E., Manners, C.R., Wayne, R.P., Shallcross, D.E., 2003. Kinetic study of the reactions of $\mathrm{OH}$ with the simple alkyl iodides: $\mathrm{CH}_{3} \mathrm{I}, \mathrm{C}_{2} \mathrm{H}_{5} \mathrm{I}, 1$ $\mathrm{C}_{3} \mathrm{H}_{7} \mathrm{I}$ and 2- $\mathrm{C}_{3} \mathrm{H}_{7} \mathrm{I}$. Atmos. Environ. 37, 1125-1133.

Cours, T., Canneaux, S., Hammaecher, C., Ribaucour, M., 2013. A theoretical study of H- and I-abstraction reactions from $\mathrm{CH}_{3} \mathrm{I}$ molecule by $\mathrm{I}\left({ }^{2} \mathrm{P}_{3 / 2}\right)$ atom and $\mathrm{IO}$ radical. Comp. Theor. Chem. 1012, 72-83. 
Cousin, F., Kissane, M.P., Girault, N., 2013. Modelling of fission-product transport in the reactor coolant system. Ann. Nucl. Energy 61, 135-142.

Cruickshank, F.R., Benson, S.W., 1969a. Carbon-hydrogen bond dissociation energy in methanol. J. Phys. Chem. 73, 733-737.

Cruickshank, F.R., Benson, S.W., 1969b. The carbon-hydrogen bond strength in dimethyl ether and some properties of iodomethyl methyl ether. Int. J. Chem. Kinet. 1, 381-390.

Cruickshank, F.R., Benson, S.W., 1969c. Kinetics and mechanism of the reaction of iodine with tetrahydrofuran. Carbon-hydrogen bond dissociation energy in tetrahydrofuran. J. Am. Chem. Soc. 91, 1289-1292.

Daykin, E.P., Wine, P.H., 1990. Kinetics of the reactions of iodine monoxide radicals with nitric oxide and nitrogen dioxide. J. Phys. Chem. 94, 4528-4535.

Diao, G., Chu, L.T., 2001. A kinetic study of the reaction of $\mathrm{NO}_{2}$ with HI over the temperature range 278 to 333 K. Phys. Chem. Chem. Phys. 3, 1622-1630.

Dillon, T.J., Tucceri, M.E., Crowley, J.N., 2006. Laser induced fluorescence studies of iodine oxide chemistry Part II. The reactions of IO with $\mathrm{CH}_{3} \mathrm{O}_{2}, \mathrm{CF}_{3} \mathrm{O}_{2}$ and $\mathrm{O}_{3}$. Phys. Chem. Chem. Phys. 8, 5185-5198.

Dillon, T.J., Tucceri, M.E., Sander, R., Crowley, J.N., 2008. LIF studies of iodine oxide chemistry Part 3. Reactions $\mathrm{IO}+\mathrm{NO}_{3} \rightarrow \mathrm{OIO}+\mathrm{NO}_{2}, \mathrm{I}+\mathrm{NO}_{3} \rightarrow \mathrm{IO}+\mathrm{NO}_{2}$, and $\mathrm{CH}_{2} \mathrm{I}$ $+\mathrm{O}_{2} \rightarrow$ (products): implications for the chemistry of the marine atmosphere at night. Phys. Chem. Chem. Phys. 10, 1540-1554.

Drougas, E., Kosmas, A.M., 2007. Ab Initio Characterization of $\left(\mathrm{CH}_{3} \mathrm{IO}_{3}\right)$ Isomers and the $\mathrm{CH}_{3} \mathrm{O}_{2}+\mathrm{IO}$ Reaction Pathways. J. Phys. Chem. A 111, 3402-3408.

Enami, S., Ueda, J., Goto, M., Nakano, Y., Aloisio, S., Hashimoto, S., Kawasaki, M., 2004. Formation of Iodine Monoxide Radical from the Reaction of $\mathrm{CH}_{2} \mathrm{I}$ with $\mathrm{O}_{2}$. J. Phys. Chem. A 108, 6347-6350. 
Enami, S., Yamanaka, T., Hashimoto, S., Kawasaki, M., Nakano, Y., Ishiwata, T., 2006. Kinetic Study of IO Radical with $\mathrm{RO}_{2}\left(\mathrm{R}=\mathrm{CH}_{3}, \mathrm{C}_{2} \mathrm{H}_{5}\right.$, and $\left.\mathrm{CF}_{3}\right)$ Using Cavity RingDown Spectroscopy. J. Phys. Chem. A 110, 9861-9866.

Eskola, A.J., Wojcik-Pastuszka, D., Ratajczak, E., Timonen, R.S., 2006. Kinetics of the Reactions of $\mathrm{CH}_{2} \mathrm{I}, \mathrm{CH}_{2} \mathrm{Br}$, and $\mathrm{CHBrCl}$ Radicals with $\mathrm{NO}_{2}$ in the Temperature Range 220-360 K. J. Phys. Chem. A 110, 12177-12183.

Fahr, A., Nayak, A.K., Kurylo, M.J., 1995. The ultraviolet absorption cross sections of $\mathrm{CH}_{3} \mathrm{I}$ temperature dependent gas and liquid phase measurements. Chem. Phys. 197, 195203.

Flowers, M.C., Benson, S.W., 1963. Kinetics of the Gas-Phase Reaction of $\mathrm{CH}_{3} \mathrm{I}$ with HI. J. Chem. Phys. 38, 882-889.

Forte, E., Hippler, H., Van Den Bergh, H., 1981. INO thermodynamic properties and ultraviolet spectrum. Int. J. Chem. Kinet. 13, 1227-1233.

Fortin, C., Khanniche, S., Khiri, D., Fèvre-Nollet, V., Lebègue, P., Cousin, F., Černušák, I., Louis, F., 2018. Reactivity of Hydrogen Peroxide with Br and I Atoms. J. Phys. Chem. A $122,1053-1063$.

Furneaux, K.L., Whalley, L.K., Heard, D.E., Atkinson, H.M., Bloss, W.J., Flynn, M.J., Gallagher, M.W., Ingham, T., Kramer, L., Lee, J.D., Leigh, R., McFiggans, G.B., Mahajan, A.S., Monks, P.S., Oetjen, H., Plane, J.M.C., Whitehead, J.D., 2010. Measurements of iodine monoxide at a semi polluted coastal location. Atmos. Chem. Phys. 10, 3645-3663.

Furuyama, S., Golden, D.M., Benson, S.W., 1969a. Kinetic studies of the reactions $\mathrm{CH}_{2} \mathrm{I}_{2}+$ $\mathrm{HI}=\mathrm{CH}_{3} \mathrm{I}+\mathrm{I}_{2}$ and $2 \mathrm{CH}_{3} \mathrm{I}=\mathrm{CH}_{4}+\mathrm{CH}_{2} \mathrm{I}_{2}$ the heat of formation of the iodomethyl radical. Int. J. Chem. Kinet. 1, 283-296. 
Furuyama, S., Golden, D.M., Benson, S.W., 1969b. Kinetic Study of the Reaction $\mathrm{CHI}_{3}+\mathrm{HI}$ $=\mathrm{CH}_{2} \mathrm{I}_{2}+\mathrm{I}_{2}$. A Summary of Thermochemical Properties of Halomethanes and Halomethyl Radicals. J. Am. Chem. Soc. 91, 7564-7569.

Furuyama, S., Golden, D.M., Benson, S.W., 1969c. Kinetics of the pyrolysis of 1,2diiodoethylene. Int. J. Chem. Kinet. 1, 147-156.

Furuyama, S., Golden, D.M., Benson, S.W., 1970a. Kinetic study of the gas-phase reaction c$\mathrm{C}_{5} \mathrm{H}_{8}+\mathrm{I}_{2}=\mathrm{c}-\mathrm{C}_{5} \mathrm{H}_{6}+2 \mathrm{HI}$. The heat of formation and the stabilization energy of the cyclopentyl radical. Int. J. Chem. Kinet. 2, 93-99.

Furuyama, S., Golden, D.M., Benson, S.W., 1970b. Kinetic study of the gas-phase reaction c$\mathrm{C}_{5} \mathrm{H}_{10}+\mathrm{I}_{2}=\mathrm{c}-\mathrm{C}_{5} \mathrm{H}_{8}+2 \mathrm{HI}$ the heat of formation of cyclopentyl radical. Int. J. Chem. Kinet. 2, 83-92.

Furuyama, S., Golden, D.M., Benson, S.W., 1971. The kinetics and thermochemistry of the gas phase reaction of cyclopentadiene and iodine. Relevance to the heat of formation of cyclopentadienyl iodide and cyclopentadienyl radical. Int. J. Chem. Kinet. 3, 237248.

Gai, Y., Ge, M., Wang, W., 2009. Kinetic studies of $\mathrm{O}_{3}$ reactions with 3-bromopropene and 3iodopropene in the temperature range 288-328 K. Atmos. Environ. 43, 3467-3471.

Galvez, O., Gómez Martin, J.C., Gomez, P.C., Saiz-Lopez, A., Pacios, L.F., 2013. A theoretical study on the formation of iodine oxide aggregates and monohydrates. Phys. Chem. Chem. Phys. 15, 15572-15583.

Gao, Y., Fessel, K., McLeod, C., Marshall, P., 2008. A kinetic study of the reaction of atomic hydrogen with iodobenzene. Chem. Phys. Lett. 451, 8-13.

Garrett, B.C., Truhlar, D.G., 1979. Generalized transition state theory. Classical mechanical theory and applications to collinear reactions of hydrogen molecules. J. Phys. Chem. $83,1052-1079$. 
Gawler, K.S., Boakes, G., Rowley, D.M., 2003. Kinetic studies of the gas phase $\mathrm{INO}_{2}$ selfreaction. Phys. Chem. Chem. Phys. 5, 3632-3637.

Gilles, M.K., Turnipseed, A.A., Talukdar, R.K., Rudich, Y., Villalta, P.W., Huey, L.G., Burkholder, J.B., Ravishankara, A.R., 1996. Reactions of $\mathrm{O}\left({ }^{3} \mathrm{P}\right)$ with Alkyl Iodides: Rate Coefficients and Reaction Products. J. Phys. Chem. 100, 14005-14015.

Girault, N., Bosland, L., Dickinson, S., Funke, F., Güntay, S., Herranz, L.E., Powers, D., 2012. LWR severe accident simulation: Iodine behaviour in FPT2 experiment and advances on containment iodine chemistry. Nucl. Eng. Des. 243, 371-392.

Golden, D.M., Benson, S.W., 1969. Free-radical and molecule thermochemistry from studies of gas-phase iodine-atom reactions. Chem. Rev. 69, 125-134.

Golden, D.M., Rodgers, A.S., Benson, S.W., 1966. The Kinetics and Mechanism of the Reaction $\mathrm{I}_{2}+\mathrm{C}_{3} \mathrm{H}_{6}=\mathrm{C}_{3} \mathrm{H}_{5} \mathrm{I}+\mathrm{HI}$ and the Heat of Formation of the Allyl Radical. J. Am. Chem. Soc. 88, 3196-3198.

Gómez Martin, J.C., Galvez, O., Baeza-Romero, M.T., Ingham, T., Plane, J.M.C., Blitz, M.A., 2013. On the mechanism of iodine oxide particle formation. Phys. Chem. Chem. Phys. 15, 15612-15622.

Gómez Martín, J.C., Spietz, P., Burrows, J.P., 2007. Kinetic and Mechanistic Studies of the $\mathrm{I}_{2} / \mathrm{O}_{3}$ Photochemistry. J. Phys. Chem. A 111, 306-320.

Hammaecher, C., Canneaux, S., Louis, F., Cantrel, L., 2011. A Theoretical Study of the HAbstraction Reactions from HOI by Moist Air Radiolytic Products ( $\mathrm{H}, \mathrm{OH}$, and $\mathrm{O}$ $\left.\left({ }^{3} \mathrm{P}\right)\right)$ and Iodine Atoms $\left({ }^{2} \mathrm{P}_{3 / 2}\right)$. J. Phys. Chem. A 115, 6664-6674.

Hartley, D.B., Benson, S.W., 1963. Kinetics of the Reaction of HI with Ethyl Iodide and the Heat of Formation of the Ethyl Radical. J. Chem. Phys. 39, 132-137. 
Harwood, M.H., Burkholder, J.B., Hunter, M., Fox, R.W., Ravishankara, A.R., 1997. Absorption Cross Sections and Self-Reaction Kinetics of the IO Radical. J. Phys. Chem. A 101, 853-863.

Hayes, D.M., Strong, R.L., 1986. Secondary reactions following flash photodissociation of iodoethane and 1-iodopropane. J. Phys. Chem. 90, 6305-6309.

Hippler, H., Luther, K., Troe, J., 1973. Untersuchung der Rekombination von Jodatomen in stark komprimierten Gasen und in Flüssigkeiten. Ber. Bunsegenges. Phys. Chem. 77, 1104-1114.

Hippler, H., Riedl, A., Troe, J., Willner, J., 1991. Thermal Decomposition of t-Butyl Bromide and t-Butyl Iodide in Shock Waves. Z. Phys. Chem. 171, 161.

Hoffmann, T., O'Dowd, C.D., Seinfeld, J.H., 2001. Iodine oxide homogeneous nucleation: An explanation for coastal new particle production. Geophys. Res. Lett. 28, 1949-1952.

Holmes, J.L., Maccoll, A., 1963. 1127. Gas-phase eliminations. Part IV. The pyrolyses of isopropyl and s-butyl iodide. J. Chem. Soc., 5919-5929.

Hunter, T.F., Kristjansson, K.S., 1982. Optoacoustic method of measuring reaction rates of the radicals $\mathrm{CH}_{3}, \mathrm{CD}_{3}, \mathrm{C}_{2} \mathrm{H}_{5}$ and $\mathrm{CH}_{2} \mathrm{I}$ with $\mathrm{I}$ and $\mathrm{I}_{2}$. J. Chem. Soc. Faraday Trans. 2: Molecular and Chemical Physics 78, 2067-2076.

IAEA, 2006. International Atomic Energy Agency, Environmental Consequences of the Chernobyl Accident and their Remediation: Twenty Years of Experience, Radiological Assessment Reports Series $N^{\circ} 8$, Vienna.

Ingham, T., Cameron, M., Crowley, J.N., 2000. Photodissociation of IO (355 nm) and OIO (532 nm): Quantum Yields for $\mathrm{O}\left({ }^{3} \mathrm{P}\right)$ and $\mathrm{I}\left({ }^{2} \mathrm{P}_{\mathrm{J}}\right)$ Production. J. Phys. Chem. A 104, 8001-8010.

IUPAC, 2007, Task Group on Atmospheric Chemical Kinetic Data Evaluation, http://www.iupac-kinetic.ch.cam.ac.uk/ 
Jacob, D., 1999. Introduction to atmospheric chemistry. Princeton University Press.

Jenkin, M.E., Cox, R.A., Hayman, G.D., 1991. Kinetics of the reaction of IO radicals with $\mathrm{HO}_{2}$ radicals at $298 \mathrm{~K}$. Chem. Phys. Lett. 177, 272-278.

Jenkin, M.E., Cox, R.A., Mellouki, A., Le Bras, G., Poulet, G., 1990. Kinetics of the reaction of iodine atoms with hydroperoxy radicals. J. Phys. Chem. 94, 2927-2934.

Jenkin, M.E., Murrells, T.P., Shalliker, S.J., Hayman, G.D., 1993. Kinetics and product study of the self-reactions of allyl and allyl peroxy radicals at 296 K. J. Chem. Soc. Faraday Trans. 89, 433-446.

Joseph, D.M., Ashworth, S.H., Plane, J.M.C., 2007. On the photochemistry of $\mathrm{IONO}_{2}$ : absorption cross section (240-370 nm) and photolysis product yields at $248 \mathrm{~nm}$. Phys. Chem. Chem. Phys. 9, 5599-5607.

Kaltsoyannis, N., Plane, J.M.C., 2008. Quantum chemical calculations on a selection of iodine-containing species ( $\mathrm{IO}, \mathrm{OIO}, \mathrm{INO}_{3},(\mathrm{IO})_{2}, \mathrm{I}_{2} \mathrm{O}_{3}, \mathrm{I}_{2} \mathrm{O}_{4}$ and $\left.\mathrm{I}_{2} \mathrm{O}_{5}\right)$ of importance in the atmosphere. Phys. Chem. Chem. Phys. 10, 1723-1733.

Katata, G., Chino, M., Kobayashi, T., Terada, H., Ota, M., Nagai, H., Kajino, M., Draxler, R., Hort, M.C., Malo, A., Torii, T., Sanada, Y., 2015. Detailed source term estimation of the atmospheric release for the Fukushima Daiichi Nuclear Power Station accident by coupling simulations of an atmospheric dispersion model with an improved deposition scheme and oceanic dispersion model. Atmos. Chem. Phys. 15, 1029-1070.

Khanniche, S., Louis, F., Cantrel, L., Černušák, I., 2016a. Computational study of the $\mathrm{I}_{2} \mathrm{O}_{5}+$ $\mathrm{H}_{2} \mathrm{O}=2 \mathrm{HOIO}_{2}$ gas-phase reaction. Chem. Phys. Lett. 662, 114-119.

Khanniche, S., Louis, F., Cantrel, L., Černušák, I., 2016b. A Density Functional Theory and ab Initio Investigation of the Oxidation Reaction of $\mathrm{CO}$ by IO Radicals. J. Phys. Chem. A 120, 1737-1749. 
Khanniche, S., Louis, F., Cantrel, L., Černušák, I., 2017. Investigation of the Reaction Mechanism and Kinetics of Iodic Acid with $\mathrm{OH}$ Radical Using Quantum Chemistry. ACS Earth Space Chem. 1, 227-235.

Kim, Y.S., Kang, W.K., Kim, D.-C., Jung, K.-H., 1997. Photodissociation of tert-Butyl Iodide at 277 and $304 \mathrm{~nm}$ : Evidence for Direct and Indirect Dissociation in A-Band Photolysis of Alkyl Iodide. J. Phys. Chem. A 101, 7576-7581.

Knox, J.H., Musgrave, R.G., 1967. Iodination of alkanes : ethane, propane and isobutane. Trans. Faraday Soc. 63, 2201-2216.

Korsakissok, I., Mathieu, A., Didier, D., 2013. Atmospheric dispersion and ground deposition induced by the Fukushima Nuclear Power Plant accident: A local-scale simulation and sensitivity study. Atmos. Environ. 70, 267-279.

Kuhn, M., Builtjes, P.J.H., Poppe, D., Simpson, D., Stockwell, W.R., Andersson-Sko“ld, Y., Baart, A., Das, M., Fiedler, F., Hov, Ø., Kirchner, F., Makar, P.A., Milford, J.B., Roemer, M.G.M., Ruhnke, R., Strand, A., Vogel, B., Vogel, H., 1998. Intercomparison of the gas-phase chemistry in several chemistry and transport models. Atmos. Environ. 32, 693-709.

Kumaran, S.S., Su, M.C., Lim, K.P., Michael, J.V., 1996. The thermal decomposition of $\mathrm{C}_{2} \mathrm{H}_{5}$ I. Symp. (Int.) Comb. 26, 605-611.

Kumaran, S.S., Su, M.C., Michael, J.V., 1997. Thermal decomposition of iodobenzene using I-atom absorption. Chem. Phys. Lett. 269, 99-106.

Kürten, A., Bergen, A., Heinritzi, M., Leiminger, M., Lorenz, V., Piel, F., Simon, M., Sitals, R., Wagner, A.C., Curtius, J., 2016. Observation of new particle formation and measurement of sulfuric acid, ammonia, amines and highly oxidized organic molecules at a rural site in central Germany. Atmos. Chem. Phys. 16, 12793-12813. 
Lancar, I.T., Mellouki, A., Poulet, G., 1991. Kinetics of the reactions of hydrogen iodide with hydroxyl and nitrate radicals. Chem. Phys. Lett. 177, 554-558.

Larin, I.K., Nevovhai, D.V., Spasskii, A.I., Trofimova, E.F.M., Turkin, L.E., 1999. Kinet. Catal. 40, 487.

Lebel, L.S., Dickson, R.S., Glowa, G.A., 2016. Radioiodine in the atmosphere after the Fukushima Daïchi nuclear accident. J. Environ. Radioact. 151, 82-93.

Leplat, N., Federič, J., Šulková, K., Sudolská, M., Louis, F., Černušák, I., Rossi, M.J., 2015. The Kinetics of the Reaction $\mathrm{C}_{2} \mathrm{H}_{5} \bullet+\mathrm{HI} \rightarrow \square_{2} \mathrm{H}_{6}+\mathrm{I} \bullet$ over an Extended Temperature Range (213-623 K): Experiment and Modeling. Z. Phys. Chem. 229, 1475.

Leplat, N., Rossi, M.J., 2014a. The Measurement of the Rate Parameters for the Reactions i$\mathrm{C}_{3} \mathrm{H}_{7}$ and $\mathrm{n}-\mathrm{C}_{3} \mathrm{H}_{7}+\mathrm{HI} \rightarrow \mathrm{C}_{3} \mathrm{H}_{8}+\mathrm{I} \bullet$ over the Temperature Range 293-623 K: Implications for the Standard Heat of Formation of the Propyl Radicals. Int. J. Chem. Kinet. 46, 305-320.

Leplat, N., Rossi, M.J., 2014b. The Reinvestigation of the Kinetics of the Metathesis Reactions $\mathrm{t}_{-} \mathrm{C}_{4} \mathrm{H}_{9} \bullet+\mathrm{HBr}(\mathrm{HI}) \rightarrow \mathrm{i}-\mathrm{C}_{4} \mathrm{H}_{10}+\mathrm{Br} \bullet(\mathrm{I} \bullet)$ and of the $\mathrm{t}_{-} \mathrm{C}_{4} \mathrm{H}_{9} \bullet$ Free Radical Thermochemistry. J. Phys. Chem. A 118, 5135-5148.

Lifshitz, A., Tamburu, C., Dubnikova, F., 2008. Reactions of 1-naphthyl radicals with ethylene. Single pulse shock tube experiments, quantum chemical, transition state theory, and multiwell calculations. J. Phys. Chem. A 112, 925-933.

Lorenz, K., Wagner, H.G., Zellner, R., 1979. Rate measurements for the reactions $\mathrm{H}+\mathrm{I}_{2} \rightarrow$ $\mathrm{HI}+\mathrm{I}$ and $\mathrm{H}+\mathrm{HI} \rightarrow \mathrm{H}_{2}+\mathrm{I}$ by Lyman- $\alpha$-fluorescence. Ber. Bunsegenges. Phys. Chem. 83, 556-560.

Louis, F., Černušák, I., Canneaux, S., Mečiarová, K., 2011. Atmospheric reactivity of $\mathrm{CH}_{3} \mathrm{I}$ and $\mathrm{CH}_{2} \mathrm{I}_{2}$ with $\mathrm{OH}$ radicals: A comparative study of the $\mathrm{H}$ - versus I-abstraction. Comput. Theoret. Chem. 965, 275-284. 
Maguin, F., Mellouki, A., Laverdet, G., Poulet, G., Le Bras, G., 1991. Kinetics of the reactions of the IO radical with dimethyl sulfide, methanethiol, ethylene, and propylene. Int. J. Chem. Kinet. 23, 237-245.

Mahajan, A.S., Oetjen, H., Saiz-Lopez, A., Lee, J.D., McFiggans, G.B., Plane, J.M.C., 2009. Reactive iodine species in a semi-polluted environment. Geophys. Res. Lett. 36, L16803.

Manion, J.A., Huie, R.E., Levin, R.D., Burgess Jr., D.R., Orkin, V.L., Tsang, W., McGivern, W.S., Hudgens, J.W., Knyazev, V.D., Atkinson, D.B., Chai, E., Tereza, A.M., Lin, C.Y., Allison, T.C., Mallard, W.G., Westley, F., Herron John, T., Hampson, R.F., Frizzell, D.H., 2015, NIST Chemical Kinetics Database, Version 7.0 (Web Version), Release 1.6.8, Dataversion 2015.09, Gaithersburg, Maryland, 20899-8320, http://kinetics.nist.gov

Martin, R.M., Willard, J.E., 1964. Reactions of Photochemically Produced Hot Hydrogen Atoms. I. Photolysis of HI at 1849 Å. J. Chem. Phys. 40, 2999-3007.

Martinez, E., Cabañas, B., Aranda, A., Martin, P., Wayne, R.P., 1996. Kinetic study of the reactions of $\mathrm{NO}_{3}$ with 3-chloropropene, 3-bromopropene and 3-iodopropene using LIF detection. J. Chem. Soc. Faraday Trans. 92, 4385-4389.

McFiggans, G., Bale, C.S.E., Ball, S.M., Beames, J.M., Bloss, W.J., Carpenter, L.J., Dorsey, J., Dunk, R., Flynn, M.J., Furneaux, K.L., Gallagher, M.W., Heard, D.E., Hollingsworth, A.M., Hornsby, K., Ingham, T., Jones, C.E., Jones, R.L., Kramer, L.J., Langridge, J.M., Leblanc, C., LeCrane, J.P., Lee, J.D., Leigh, R.J., Longley, I., Mahajan, A.S., Monks, P.S., Oetjen, H., Orr-Ewing, A.J., Plane, J.M.C., Potin, P., Shillings, A.J.L., Thomas, F., von Glasow, R., Wada, R., Whalley, L.K., Whitehead, J.D., 2010. Iodine-mediated coastal particle formation: an overview of the Reactive 
Halogens in the Marine Boundary Layer (RHaMBLe) Roscoff coastal study. Atmos. Chem. Phys. 10, 2975-2999.

McFiggans, G., Coe, H., Burgess, R., Allan, J., Cubison, M., Alfarra, M.R., Saunders, R., Saiz-Lopez, A., Plane, J.M.C., Wevill, D., Carpenter, L., Rickard, A.R., Monks, P.S., 2004. Direct evidence for coastal iodine particles from Laminaria macroalgae linkage to emissions of molecular iodine. Atmos. Chem. Phys. 4, 701-713.

McFiggans, G., Plane John, M.C., Allan Beverley, J., Carpenter Lucy, J., Coe, H., O'Dowd, C., 2000. A modeling study of iodine chemistry in the marine boundary layer. J. Geophys. Res.: Atm. 105, 14371-14385.

McMillen, D.F., Golden, D.M., Benson, S.W., 1971. Kinetics of the gas-phase reaction of cyclopropylcarbinyl iodide and hydrogen iodide. Heat of formation and stabilization energy of the cyclopropylcarbinyl radical. Int. J. Chem. Kinet. 3, 359-374.

McMillen, D.F., Golden, D.M., Benson, S.W., 1972. The rate of the gas phase iodination of cyclobutane. The heat of formation of the cyclobutyl radical. Int. J. Chem. Kinet. 4, 487-495.

Mečiarová, K., Šulka, M., Canneaux, S., Louis, F., Černušák, I., 2011. A theoretical study of the kinetics of the forward and reverse reactions $\mathrm{HI}+\mathrm{CH}_{3}=\mathrm{I}+\mathrm{CH}_{4}$. Chem. Phys. Lett. 517, 149-154.

Menut, L., Bessagnet, B., Khvorostyanov, D., Beekmann, M., Blond, N., Colette, A., Coll, I., Curci, G., Foret, G., Hodzic, A., Mailler, S., Meleux, F., Monge, J.L., Pison, I., Siour, G., Turquety, S., Valari, M., Vautard, R., Vivanco, M.G., 2013. CHIMERE 2013: a model for regional atmospheric composition modelling. Geosci. Model Dev. 6, 9811028.

Michael, J.V., Kumaran, S.S., Su, M.C., Lim, K.P., 2000. Thermal rate constants over thirty orders of magnitude for the $\mathrm{I}+\mathrm{H}_{2}$ reaction. Chem. Phys. Lett. 319, 99-106. 
Mössinger, J.C., Rowley, D.M., Cox, R.A., 2002. The UV-visible absorption cross-sections of $\mathrm{IONO}_{2}$. Atmos. Chem. Phys. 2, 227-234.

Mössinger, J.C., Shallcross, D.E., Cox, R.A., 1998. UV-VIS absorption cross-sections and atmospheric lifetimes of $\mathrm{CH}_{2} \mathrm{Br}_{2}, \mathrm{CH}_{2} \mathrm{I}_{2}$ and $\mathrm{CH}_{2}$ BrI. J. Chem. Soc. Faraday Trans. 94, 1391-1396.

Mueller-Markgraf, W., Troe, J., 1988. Thermal decomposition of benzyl iodide and of benzyl radicals in shock waves. J. Phys. Chem. 92, 4899-4905.

Nakano, Y., Ukeguchi, H., Ishiwata, T., 2006. Rate constant of the reaction of $\mathrm{NO}_{3}$ with $\mathrm{CH}_{2} \mathrm{I}_{2}$ measured with use of cavity ring-down spectroscopy. Chem. Phys. Lett. 430, 235-239.

Nakano, Y., Ukeguchi, H., Ishiwata, T., Kanaya, Y., Tachikawa, H., Ikeda, A., Sakaki, S., Kawasaki, M., 2008. Study of the temperature dependence of the reaction of $\mathrm{NO}_{3}$ with $\mathrm{CH}_{3} \mathrm{I}$ and the estimation of its impact on atmospheric iodine chemistry. Bull. Chem. Soc. Jpn. 81, 938-946.

Nangia, P.S., Benson, S.W., 1964. Kinetics of the Gas-Phase Addition of HI to Butene-2. J. Chem. Phys. 41, 530-537.

O'Neal, E., Benson, S.W., 1962a. Photolysis of Acetone in the Presence of HI and the Decomposition of the Acetyl Radical. J. Chem. Phys. 36, 2196-2203.

O'Neal, H.E., Benson, S.W., 1962b. Kinetics of the Reaction between Acetyl Iodide and HI and the Heat of Formation of the Acetyl Radical. J. Chem. Phys. 37, 540-543.

Ogg, R.A., Priest, W.J., 1939. Kinetics of the Vapor Phase Reaction of Cyclopropane with Iodine. J. Chem. Phys. 7, 736-748.

Ordóñez, C., Lamarque, J.F., Tilmes, S., Kinnison, D.E., Atlas, E.L., Blake, D.R., Sousa Santos, G., Brasseur, G., Saiz-Lopez, A., 2012. Bromine and iodine chemistry in a 
global chemistry-climate model: description and evaluation of very short-lived oceanic sources. Atmos. Chem. Phys. 12, 1423-1447.

Pechtl, S., Lovejoy, E.R., Burkholder, J.B., von Glasow, R., 2006. Modeling the possible role of iodine oxides in atmospheric new particle formation. Atmos. Chem. Phys. 6, 505523.

Phillips, D.L., Myers, A.B., Valentini, J.J., 1992. Investigation of solvation effects on shorttime photodissociation dynamics of alkyl iodides. J. Phys. Chem. 96, 2039-2044.

Plane, J.M.C., Joseph, D.M., Allan, B.J., Ashworth, S.H., Francisco, J.S., 2006. An Experimental and Theoretical Study of the Reactions $\mathrm{OIO}+\mathrm{NO}$ and $\mathrm{OIO}+\mathrm{OH}$. J. Phys. Chem. A 110, 93-100.

Rattigan, O.V., Shallcross, D.E., Cox, R.A., 1997. UV absorption cross-sections and atmospheric photolysis rates of $\mathrm{CF}_{3} \mathrm{I}, \mathrm{CH}_{3} \mathrm{I}, \mathrm{C}_{2} \mathrm{H}_{5} \mathrm{I}$ and $\mathrm{CH}_{2} \mathrm{ICl}$. J. Chem. Soc. Faraday Trans. 93, 2839-2846.

Rebbert, R.E., Lias, S.G., Ausloos, P., 1973. Photolysis of alkyl iodides at $147.0 \mathrm{~nm}$. The reaction $\mathrm{H}+\mathrm{C}_{\mathrm{n}} \mathrm{H}_{2 \mathrm{n}+1} \mathrm{I} \rightarrow \mathrm{HI}+\mathrm{C}_{\mathrm{n}} \mathrm{H}_{2 \mathrm{n}+1}$. Int. J. Chem. Kinet. 5, 893-908.

Rissanen, M.P., Rose, C., Iyer, S., He, X., Tham, Y.J., Lamolahti, J., Wimmer, D., Metzger, J.-M., Magand, O., Koenig, T.K., Finkenzeller, H., Colomb, A., Sellegri, K., Verreyken, B., Amelynck, C., Stavrakou, T., Schoon, N., Tulet, P., Cammas, J.-P., Volkamer, R., Brioude, J., 2019. Iodic acid and new particle formation observed at the high-altitude station of Maïdo (Réunion), EGU. Geophysical Research Abstracts, Vienna, pp. EGU2019-17451-17452.

Robaugh, D., Tsang, W., 1986. Thermal decomposition of phenyl iodide and o-iodotoluene. J. Phys. Chem. 90, 5363-5367. 
Rodgers, A.S., Golden, D.M., Benson, S.W., 1967. Kinetics of the reaction of iodobenzene and hydrogen iodide. Heat of formation of the phenyl radical and its implications on the reactivity of benzene. J. Am. Chem. Soc. 89, 4578-4583.

Roehl, C.M., Burkholder, J.B., Moortgat, G.K., Ravishankara, A.R., Crutzen, P.J., 1997. Temperature dependence of UV absorption cross sections and atmospheric implications of several alkyl iodides. J. Geophys. Res. 102, 12819-12829.

Rossi, M., Golden, D.M., 1979. Absolute rate constants for metathesis reactions of allyl and benzyl radicals with hydriodic acid (hydriodic acid-d). Heat of formation of allyl and benzyl radicals. J. Am. Chem. Soc. 101, 1230-1235.

Rowley, D.M., Mössinger, J.C., Cox, R.A., Jones, R.L., 1999. The UV-Visible Absorption Cross-Sections and Atmospheric Photolysis Rate of HOI. J. Atmos. Chem. 34, 137151.

Saito, K., Tahara, H., Kondo, O., Yokubo, T., Thigashihara, T., Murakami, I., 1980. The Thermal Gas-phase Decomposition of Methyl Iodide. Bull. Chem. Soc. Jpn. 53, 13351339.

Saiz-Lopez, A., Fernandez, R.P., Ordóñez, C., Kinnison, D.E., Gómez Martín, J.C., Lamarque, J.F., Tilmes, S., 2014. Iodine chemistry in the troposphere and its effect on ozone. Atmos. Chem. Phys. 14, 13119-13143.

Saiz-Lopez, A., Plane, J.M.C., Baker, A.R., Carpenter, L.J., von Glasow, R., Gómez Martín, J.C., McFiggans, G., Saunders, R.W., 2012. Atmospheric Chemistry of Iodine. Chem. Rev. 112, 1773-1804.

Saiz-Lopez, A., Plane, J.M.C., Cuevas, C.A., Mahajan, A.S., Lamarque, J.F., Kinnison, D.E., 2016. Nighttime atmospheric chemistry of iodine. Atmos. Chem. Phys. 16, 1559315604. 
Saiz-Lopez, A., Plane, J.M.C., Mahajan, A.S., Anderson, P.S., Bauguitte, S.J.B., Jones, A.E., Roscoe, H.K., Salmon, R.A., Bloss, W.J., Lee, J.D., Heard, D.E., 2008. On the vertical distribution of boundary layer halogens over coastal Antarctica: implications for $\mathrm{O}_{3}$, $\mathrm{HO}_{\mathrm{x}}, \mathrm{NO}_{\mathrm{x}}$ and the $\mathrm{Hg}$ lifetime. Atmos. Chem. Phys. 8, 887-900.

Saiz-Lopez, A., Plane, J.M.C., McFiggans, G., Williams, P.I., Ball, S.M., Bitter, M., Jones, R.L., Hongwei, C., Hoffmann, T., 2006. Modelling molecular iodine emissions in a coastal marine environment: the link to new particle formation. Atmos. Chem. Phys. 6, 883-895.

Saiz-Lopez, A., Saunders, R.W., Joseph, D.M., Ashworth, S.H., Plane, J.M.C., 2004. Absolute absorption cross-section and photolysis rate of $\mathrm{I}_{2}$. Atmos. Chem. Phys. 4, 1443-1450.

Saunders, R.W., Plane, J.M.C., 2006. Fractal growth modelling of $\mathrm{I}_{2} \mathrm{O}_{5}$ nanoparticles. J. Aerosol Sci. 37, 1737-1749.

Saunier, O., Mathieu, A., Didier, D., Tombette, M., Quélo, D., Winiarek, V., Bocquet, M., 2013. An inverse modeling method to assess the source term of the Fukushima Nuclear Power Plant accident using gamma dose rate observations. Atmos. Chem. Phys. 13, 11403-11421.

Schmitt, G., Comes, F.J., 1980. Photolysis of $\mathrm{CH}_{2} \mathrm{I}_{2}$ and 1,1- $\mathrm{C}_{2} \mathrm{H}_{4} \mathrm{I}_{2}$ at $300 \mathrm{~nm}$. Journal of Photochemistry 14, 107-123.

Seetula, J.A., 1991. Kinetics, thermochemistry and reactivity of reactions of some polyatomic free radicals with $\mathrm{HI}, \mathrm{HBr}, \mathrm{Cl}_{2}$ and $\mathrm{Br}_{2}$, in Annales Academiae Scientiarum Fennicae, Series A2 Chemica, ed. Pub. Academia Scientiarum Fennica, Yliopistopaino, Helsinki (Finland), pp. 1-45.

Seetula, J.A., Gutman, D., 1991. Kinetics of reactions of halogenated methyl radicals with hydrogen iodide. J. Phys. Chem. 95, 3626-3630. 
Seetula, J.A., Gutman, D., 1992. Kinetics of the hydroxymethyl + hydrogen bromide and hydroxymethyl + hydrogen iodide reactions and determination of the heat of formation of hydroxymethyl. J. Phys. Chem. 96, 5401-5405.

Seetula, J.A., Russell, J.J., Gutman, D., 1990. Kinetics and thermochemistry of the reactions of alkyl radicals (methyl, ethyl, isopropyl, sec-butyl, tert-butyl) with hydrogen iodide: a reconciliation of the alkyl radical heats of formation. J. Am. Chem. Soc. 112, 13471353.

Sehested, J., Ellermann, T., Nielsen Ole, J., 1994. A spectrokinetic study of $\mathrm{CH}_{2} \mathrm{I}$ and $\mathrm{CH}_{2} \mathrm{IO}_{2}$ radicals. Int. J. Chem. Kinet. 26, 259-272.

Seitz, K., Buxmann, J., Pöhler, D., Sommer, T., Tschritter, J., Neary, T., O'Dowd, C., Platt, U., 2010. The spatial distribution of the reactive iodine species IO from simultaneous active and passive DOAS observations. Atmos. Chem. Phys. 10, 2117-2128.

Shah, D., Canosa-Mas, C.E., Hendy, N.J., Scott, M.J., Vipond, A., Wayne, R.P., 2001. Discharge-flow studies of the kinetics of the reactions of $\mathrm{CH}_{3} \mathrm{O}$ with $\mathrm{Cl}, \mathrm{Br}, \mathrm{I}, \mathrm{ClO}$, $\mathrm{BrO}$ and $\mathrm{IO}$ using laser-induced fluorescence and resonance-fluorescence detection. Phys. Chem. Chem. Phys. 3, 4932-4938.

Sheps, L., 2013. Absolute Ultraviolet Absorption Spectrum of a Criegee Intermediate $\mathrm{CH}_{2} \mathrm{OO}$. J. Phys. Chem. Lett. 4, 4201-4205.

Sheps, L., Scully, A.M., Au, K., 2014. UV absorption probing of the conformer-dependent reactivity of a Criegee intermediate $\mathrm{CH}_{3} \mathrm{CHOO}$. Phys. Chem. Chem. Phys. 16, 2670126706.

Sherwen, T., Evans, M.J., Carpenter, L.J., Andrews, S.J., Lidster, R.T., Dix, B., Koenig, T.K., Sinreich, R., Ortega, I., Volkamer, R., Saiz-Lopez, A., Prados-Roman, C., Mahajan, A.S., Ordóñez, C., 2016a. Iodine's impact on tropospheric oxidants: a global model study in GEOS-Chem. Atmos. Chem. Phys. 16, 1161-1186. 
Sherwen, T., Schmidt, J.A., Evans, M.J., Carpenter, L.J., Großmann, K., Eastham, S.D., Jacob, D.J., Dix, B., Koenig, T.K., Sinreich, R., Ortega, I., Volkamer, R., Saiz-Lopez, A., Prados-Roman, C., Mahajan, A.S., Ordóñez, C., 2016b. Global impacts of tropospheric halogens $(\mathrm{Cl}, \mathrm{Br}, \mathrm{I})$ on oxidants and composition in GEOS-Chem. Atmos. Chem. Phys. 16, 12239-12271.

Shilov, A.E., Sabirova, R.D., 1964. The mechanism of the thermal decomposition of ethyl and vinyl iodides and the simultaneous occurence of two types of primary decomposition of alkyl halides. Kinet. Catal. 5, 32-39.

Shum, L.G.S., Benson, S.W., 1983. The oxidation of HI at low temperatures and the heat of formation of $\mathrm{HO}_{2}$. Int. J. Chem. Kinet. 15, 323-339.

Sidebottom, H., Treacy, J., 1984. Reaction of methyl radicals with haloalkanes. Int. J. Chem. Kinet. 16, 579-590.

Singleton, D.L., Cvetanović, R.J., 1978. Temperature dependence of rate constants for the reactions of oxygen atoms, $\mathrm{O}\left({ }^{3} \mathrm{P}\right)$, with $\mathrm{HBr}$ and $\mathrm{HI}$. Can. J. Chem. 56, 2934-2939.

Sipilä, M., Sarnela, N., Jokinen, T., Henschel, H., Junninen, H., Kontkanen, J., Richters, S., Kangasluoma, J., Franchin, A., Peräkylä, O., Rissanen, M.P., Ehn, M., Vehkamäki, H., Kurten, T., Berndt, T., Petäjä, T., Worsnop, D., Ceburnis, D., Kerminen, V.-M., Kulmala, M., O’Dowd, C., 2016. Molecular-scale evidence of aerosol particle formation via sequential addition of $\mathrm{HIO}_{3}$. Nature 537, 532-534.

Skorobogatov, G.A., Dymov, B.P., Nedozrelova, I.V., 1994. Equilibrium constants and rate constants of $\mathrm{CX}_{3} \mathrm{I}=\mathrm{CX}_{3}+\mathrm{I}, \mathrm{CX}_{3} \mathrm{I}_{2}=\mathrm{I}_{2}+\mathrm{CX}_{3}$, where $(\mathrm{X}=\mathrm{H}, \mathrm{D})$ reactions in $300-800$ K temperature range. Zh. Org. Khim. 64, 956-965.

Solly, R.K., Benson, S.W., 1971. Kinetics of the gas-phase reaction of benzaldehyde with iodine. Heat of formation and stabilization energy of the benzoyl radical. J. Am. Chem. Soc. 93, 1592-1595. 
Solly, R.K., Golden, D.M., Benson, S., W., 1970a. Kinetics and thermochemistry of the gas phase reaction of methyl ethyl ketone with iodine. I. The resonance energy of the methylacetonyl radical. Int. J. Chem. Kinet. 2, 381-391.

Solly, R.K., Golden, D.M., Benson, S., W., 1970b. Kinetics of the gas-phase reaction of acetone with iodine: Heat of formation and stabilization energy of the acetonyl radical. Int. J. Chem. Kinet. 2, 11-21.

Sommariva, R., Bloss, W.J., von Glasow, R., 2012. Uncertainties in gas-phase atmospheric iodine chemistry. Atmos. Environ. 57, 219-232.

Stockwell, W.R., Kirchner, F., Kuhn, M., Seefeld, S., 1997. A new mechanism for regional atmospheric chemistry modeling. J. Geophys. Res.: Atm. 102, 25847-25879.

Stone, D., Sherwen, T., Evans, M.J., Vaughan, S., Ingham, T., Whalley, L.K., Edwards, P.M., Read, K.A., Lee, J.D., Moller, S.J., Carpenter, L.J., Lewis, A.C., Heard, D.E., 2018. Impacts of bromine and iodine chemistry on tropospheric $\mathrm{OH}$ and $\mathrm{HO}_{2}$ : comparing observations with box and global model perspectives. Atmos. Chem. Phys. 18, 35413561 .

Stone, D., Whalley, L.K., Heard, D.E., 2012. Tropospheric $\mathrm{OH}$ and $\mathrm{HO}_{2}$ radicals: field measurements and model comparisons. Chemical Society Reviews 41, 6348-6404.

Sullivan, J.H., 1959. Rates of Reaction of Hydrogen with Iodine. J. Chem. Phys. 30, 12921300.

Teranishi, H., Benson, S.W., 1963. The Kinetics of Dehydrogenation of Isobutane by Iodine and the Heat of Formation of the t-Butyl Radical. J. Am. Chem. Soc. 85, 2887-2890.

Teruel, M.A., Dillon, T.J., Horowitz, A., Crowley, J.N., 2004. Reaction of O $\left({ }^{3} \mathrm{P}\right)$ with the alkyl iodides: $\mathrm{CF}_{3} \mathrm{I}, \mathrm{CH}_{3} \mathrm{I}, \mathrm{CH}_{2} \mathrm{I}_{2}, \mathrm{C}_{2} \mathrm{H}_{5} \mathrm{I}, 1-\mathrm{C}_{3} \mathrm{H}_{7} \mathrm{I}$ and 2- $\mathrm{C}_{3} \mathrm{H}_{7} \mathrm{I}$. Phys. Chem. Chem. Phys. 6, 2172-2178. 
Tsang, W., 1964. Thermal Decomposition of Some Alkyl Halides by a Shock-Tube Method. J. Chem. Phys. 41, 2487-2494.

Tucceri, M.E., Dillon, T.J., Crowley, J.N., 2005. A laser photolysis-resonance fluorescence study of the reactions: $\mathrm{I}+\mathrm{O}_{3} \rightarrow \mathrm{IO}+\mathrm{O}_{2}, \mathrm{O}+\mathrm{I}_{2} \rightarrow \mathrm{IO}+\mathrm{I}$, and $\mathrm{I}+\mathrm{NO}_{2}+\mathrm{M} \rightarrow \mathrm{INO}_{2}$ + M at 298 K. Phys. Chem. Chem. Phys. 7, 1657-1663.

Umemoto, H., Nakagawa, S., Tsunashima, S., Sato, S., 1988. The reaction of H+HI and its isotopic variants. Chem. Phys. 124, 259-263.

van den Bergh, H., Troe, J., 1976. Kinetic and thermodynamic properties of INO and $\mathrm{INO}_{2}$ intermediate complexes in iodine recombination. J. Chem. Phys. 64, 736-742.

Vickis, A.C., 1989. Proceedings of the OECD/NEA sponsored Second CSNI Workshop on Iodine Chemistry in Reactor Safety, 1988, Available as Atomic Energy of Canada Limited Report AECL-9923 or CSNI-149, Toronto, Canada, pp. 1-329.

Vikis, A.C., MacFarlane, R., 1985. Reaction of iodine with ozone in the gas phase. J. Phys. Chem. 89, 812-815.

von Glasow, R., Crutzen, P.J., 2003. Tropospheric Halogen Chemistry, in Treatise on Geochemistry, ed. Heinrich, D.H., Karl, K.T. Pergamon, Oxford, pp. 21-64.

Wallington, T., J., Neuman, D., M., Kurylo, M., J., 1987. Kinetics of the gas phase reaction of hydroxyl radicals with ethane, benzene, and a series of halogenated benzenes over the temperature range 234-438 K. Int. J. Chem. Kinet. 19, 725-739.

Walsh, R., 1971. Kinetics of the gas-phase iodine-catalyzed isomerization of methyl acetylene and the propargyl stabilization energy. Trans. Faraday Soc. 67, 2085-2095.

Walsh, R., Benson, S.W., 1966a. Kinetics and Mechanism of the Gas Phase Reaction between Iodine and Formaldehyde and the Carbon-Hydrogen Bond Strength in Formaldehyde. J. Am. Chem. Soc. 88, 4570-4575. 
Walsh, R., Benson, S.W., 1966b. Kinetics and Mechanism of the Gas Phase Reaction between Iodine and Isopropyl Alcohol and the Tertiary Carbon-Hydrogen Bond Strength in Isopropyl Alcohol. J. Am. Chem. Soc. 88, 3480-3485.

Walsh, R., Golden, D.M., Benson, S.W., 1966. The Thermochemistry of the Gas Phase Equilibrium $\mathrm{I}_{2}+\mathrm{C}_{6} \mathrm{H}_{5} \mathrm{CH}_{3}=\mathrm{C}_{6} \mathrm{H}_{5} \mathrm{CH}_{2} \mathrm{I}+\mathrm{HI}$ and the Heat of Formation of the Benzyl Radical. J. Am. Chem. Soc. 88, 650-656.

Xerri, B., Canneaux, S., Louis, F., Trincal, J., Cousin, F., Badawi, M., Cantrel, L., 2012. Ab initio calculations and iodine kinetic modeling in the reactor coolant system of a pressurized water reactor in case of severe nuclear accident. Comp. Theor. Chem. 990, 194-208.

Yuan, J., Wells, L., Marshall, P., 1997. Kinetic Studies of the Reactions of Atomic Hydrogen with Iodoalkanes. J. Phys. Chem. A 101, 3542-3546.

Zhang, S., Strekowski, R., Bosland, L., Monod, A., Zetzsch, C., 2011a. Kinetic study of the reaction of $\mathrm{OH}$ with $\mathrm{CH}_{2} \mathrm{I}_{2}$. Phys. Chem. Chem. Phys. 13, 11671-11677.

Zhang, S., Strekowski Rafal, S., Bosland, L., Monod, A., Zetzsch, C., 2011b. Kinetic study of the reaction of $\mathrm{OH}$ with $\mathrm{CH}_{3} \mathrm{I}$ revisited. Int. J. Chem. Kinet. 43, 547-556.

Zhang, S., Strekowski, R.S., Monod, A., Bosland, L., Zetzsch, C., 2012. TemperatureDependent Kinetics Study of the Reactions of $\mathrm{OH}$ with $\mathrm{C}_{2} \mathrm{H}_{5} \mathrm{I}, \mathrm{n}-\mathrm{C}_{3} \mathrm{H}_{7} \mathrm{I}$, and iso- $\mathrm{C}_{3} \mathrm{H}_{7} \mathrm{I}$. J. Phys. Chem. A 116, 9497-9506. 


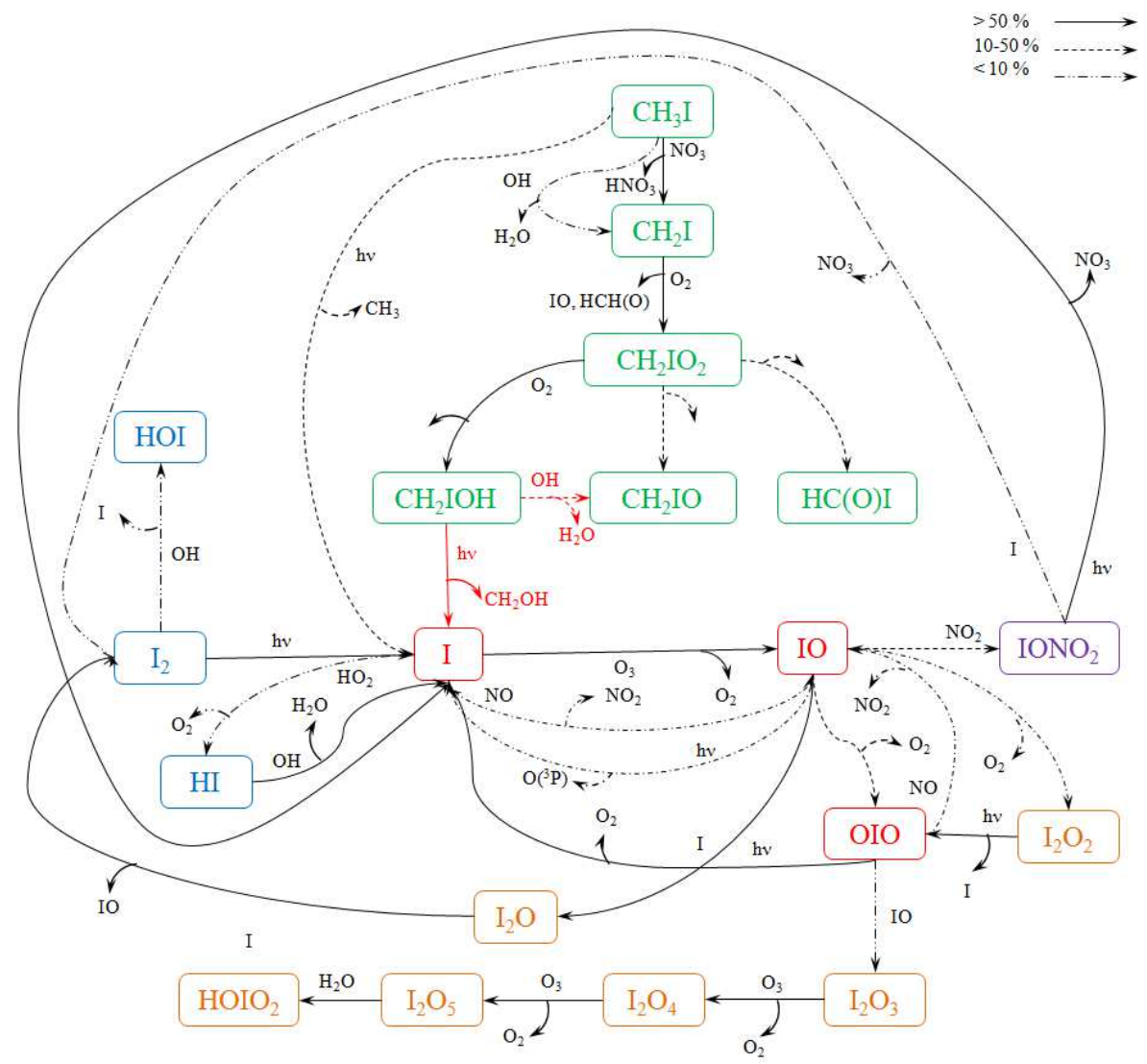

Iodine reaction scheme, 2013 August $8^{\text {th }}$, for an iodine release at 4 am $196 \mathrm{pptV}$ of $\mathrm{CH}_{3} \mathrm{I}$ 